\title{
Discrete Time Dynamic Traffic Assignment Models with Lane Reversals for Evacuation Planning
}

\author{
Yeh-Ern Poh \\ West Virginia University
}

Follow this and additional works at: https://researchrepository.wvu.edu/etd

\section{Recommended Citation}

Poh, Yeh-Ern, "Discrete Time Dynamic Traffic Assignment Models with Lane Reversals for Evacuation Planning" (2013). Graduate Theses, Dissertations, and Problem Reports. 3401.

https://researchrepository.wvu.edu/etd/3401

This Thesis is protected by copyright and/or related rights. It has been brought to you by the The Research Repository @ WVU with permission from the rights-holder(s). You are free to use this Thesis in any way that is permitted by the copyright and related rights legislation that applies to your use. For other uses you must obtain permission from the rights-holder(s) directly, unless additional rights are indicated by a Creative Commons license in the record and/ or on the work itself. This Thesis has been accepted for inclusion in WVU Graduate Theses, Dissertations, and Problem Reports collection by an authorized administrator of The Research Repository @ WVU. For more information, please contact researchrepository@mail.wvu.edu. 


\title{
Discrete Time Dynamic Traffic Assignment Models with Lane Reversals for Evacuation Planning
}

\author{
by \\ Yeh-Ern Poh \\ Thesis submitted to the \\ College of Engineering and Mineral Resources \\ at West Virginia University \\ in partial fulfillment of the requirements \\ for the degree of \\ Master of Science \\ in \\ Industrial Engineering \\ David R. Martinelli, Ph.D. \\ Robert C. Creese, Ph.D. \\ Qipeng P. Zheng, Ph.D., Chair
}

Department of Industrial and Management Systems Engineering

Morgantown, West Virginia

2013

Keywords: Dynamic traffic assignment, lane reversal, collision avoidance

Copyright 2013 Yeh-Ern Poh 


\author{
Abstract \\ Discrete Time Dynamic Traffic Assignment Models with Lane Reversals for Evacuation \\ Planning \\ by \\ Yeh-Ern Poh \\ Master of Science in Industrial Engineering \\ West Virginia University \\ Qipeng P. Zheng, Ph.D., Chair
}

In an event of a natural or man-made disaster, an evacuation is likely to be called for to move residents away from potentially hazardous areas. Road congestion and traffic stalling is a common occurrence as residents evacuate towns and cities for safe refuges. Lane reversal, or contra-flow, is a remedy to increase outbound flow capacities from disaster areas which in turn will reduce evacuation time of evacuees during emergency situations. This thesis presents a discrete-time traffic assignment system with lane reversals which incorporates multiple sources and multiple destinations to predict optimal traffic flow at various times throughout the entire planning horizon. With the realization of lane reversals, naturally the threat of potential head-on collisions emerges. To avoid the occurrence of such situations, a collision prevention constraint is introduced to limit directional flow on lanes based on departure time.

This model belongs to the class of dynamic traffic assignment (DTA) problems. Initially the model was formulated as a discrete-time system optimum dynamic traffic assignment (DTA-SO) problem, which is a mixed integer nonlinear programming problem. Through various proven theorems, a linearized upper bound was derived that is able to approximate the original problem with very high precision. The result is an upper bound mixed integer linear programming problem (DTA-UB). The discrete-time DTA model is suitable for evacuation planning because the model is able to take care of dynamic demands, and temporal flow assignment. Also, simultaneous route and departure is assumed and an appropriate travel time function is used to approximate the minimum and maximum travel time on an arc.

This thesis discusses the different attributes that relates to Dynamic Traffic Assignment. DTA model properties and formulation methodology are also expounded upon. A model analysis that breaks down each output into individual entities is provided to further understand the computational results of small networks. A no reversal DTA-UB model (NRDTA-UB) is formulated and its computational results are compared to DTAUB. Through the extensive computational results, DTA-UB is proven to obtain much better results than NRDTA-UB despite having longer solving time. This is a step toward realizing the supremacy of having lane reversals in a real-life evacuation scenario. 
To my father and mother

\author{
Boon Sing Poh \\ Good Cam Ng \\ and my brothers \\ Yeh Han Poh \\ Yeh Tze Poh \\ Yeh Chuin Poh
}

for their love and continuous support. 


\section{Acknowledgements}

First and foremost, I would like to thank my research advisor, Dr. Qipeng P. Zheng for introducing me to the field of transportation and optimization. His guidance, supervision, and counsel throughout my masters training are only a fraction of the things that I have to thank him for. My utmost gratitude extends to the people in Dr. Zheng's research group: Yuping Huang, Yi Fang, Daniel Simmons, Anees Rahil, and Yixin Justin Du. In one way or another, you have all helped me tremendously in the completion of this thesis. This thesis and the culmination of my masters degree will not be possible without the help of Yuping Huang especially for tirelessly spending hours and hours going through my mathematical model and $\mathrm{C}++$ coding to correct mistakes and to debug errors. Thank you for your support and invaluable advice that has helped me in my understanding of Dynamic Traffic Assignment. Special thanks also goes to Yi Fang, whose gentle guidance has compelled me to strife for better in my computer coding.

I would also like to thank the rest of my committee members, Dr. Robert Creese and Dr. David Martinelli, for their guidance and feedback, and also for being exceedingly patient throughout the the process of completing my thesis. Heartfelt thanks goes to my collaborator, Chrysafis Vogiatzis, from the University of Florida, for his insights and coding expertise and also for helping me with the generation of results for large networks through the supercomputer at the University of Florida.

My time in Morgantown would not have been the same without the love and support from Pastor Joseph Cheng and his dear wife, Sharon Cheng. They have been a tremendous encouragement in times of need and have always been a parental figure to me. Because of moving down south of the country, their presence is greatly missed in Morgantown. To the folks up in Michigan, Uncle Chek Teck, Aunty Elsa, and the entire Pakala family, all of whom have been exceedingly generous and caring in my trips there during thanksgiving breaks. Sincerest thanks to Evelyn Tsui, for being extraordinarily patient and supportive 
of me in all my decisions throughout these past few years.

Last and most importantly, I would like to thank my family for always being a source of encouragement and support. To my father, who has been an inspiration to me since I was young. A role model I hope to emulate, both in character and in spirit. To my mother, who has been a constant comfort and always just a phone call away. A gentle soul that I can only aspire to be like. Her supportive personality and caring character guided me through thick and thin. I truly owe it to my parents for their daily thoughts and prayers throughout my university education. To my three older brothers, Yeh Han, Yeh Tze, and Yeh Chuin, despite the constant bulling from them, all of whom have kept me grounded and constantly reminded me to always keep things in perspective. Family is undeniably my chief pillar of support. 


\section{Contents}

$\begin{array}{ll}\text { Dedication } & 3\end{array}$

Acknowledgements $\quad$ iii

List of Figures $\quad$ vi

List of Tables $\quad$ viii

1 Introduction $\quad 1$

1.1 Man-made and Natural Disasters . . . . . . . . . . . . . . . . . . . 1

1.2 Emergency Evacuation Planning . . . . . . . . . . . . . . . 3

1.2.1 Related Work . . . . . . . . . . . . . . . . . . 4

1.2.2 Importance of an Emergency Evacuation Plan . . . . . . . . . . 5

1.3 Research Objective . . . . . . . . . . . . . . . . . . . 5

1.4 Thesis Organization . . . . . . . . . . . . . . . . . 6

2 Review of Emergency Evacuation Characteristics $\quad 8$

2.1 Microscopic Evacuation Planning . . . . . . . . . . . . . . . . . 9 9

2.2 Macroscopic Evacuation Planning . . . . . . . . . . . . . . . . . 11

2.3 Static Traffic Assignment . . . . . . . . . . . . . . . . . . . . 15

2.3.1 Static Traffic Assignment Limitations . . . . . . . . . . . . . . 16

2.4 Dynamic Traffic Assignment . . . . . . . . . . . . . . . . . . . 17

2.5 Dynamic Traffic Assignment for Emergency Evacuation Planning . . . . . 19

2.5.1 Simulation Approach . . . . . . . . . . . . . . . . 23

2.5.2 Analytical Approach . . . . . . . . . . . . . . . . . . . . 25

2.6 Contra-Flow / Lane Reversals . . . . . . . . . . . . . . . . . . 27

2.7 Project Significance . . . . . . . . . . . . . . . . . . . . . . . . . . 28

3 Problem Formulation $\quad 31$

3.1 Time Expanded Network . . . . . . . . . . . . . . . . . . . . . . . 32

3.2 Travel Time Function . . . . . . . . . . . . . . . . . . . . . . 34

3.3 Travel Time . . . . . . . . . . . . . . . . . . . . . . 37

3.4 Demand Generation . . . . . . . . . . . . . . . . . . . . 40

3.5 Flow Balance . . . . . . . . . . . . . . . . . . . 41

3.6 Collision Prevention . . . . . . . . . . . . . . . . . . 43 
3.7 Mathematical Formulation . . . . . . . . . . . . . . . . . 45

3.8 Model Assumptions and Limitations . . . . . . . . . . . . . . . . . . . . . 49

4 Model Analysis $\quad 51$

4.1 No Reversal Model . . . . . . . . . . . . . . . . . . . . . . . . . . 51

4.2 Computer Specifications and Software . . . . . . . . . . . . 53

4.3 Small Network: 2 Nodes . . . . . . . . . . . . . . . . . . . . . 54

4.3.1 Results for Model with Lane Reversal . . . . . . . . . . . . . . . . . 55

4.3.2 Results for Model without Lane Reversal . . . . . . . . . . . . . . . 56

4.4 Small Network: 6 Nodes . . . . . . . . . . . . . . . . . . . . . . . . 58

4.4.1 Results for Model with Lane Reversal . . . . . . . . . . . . . . . . . 60

4.4.2 Results for Model without Lane Reversal . . . . . . . . . . . . . . . 62

$5 \quad$ Numerical Results $\quad 65$

5.1 Sioux Falls Network . . . . . . . . . . . . . . . . . 65

5.1.1 Smallest Time Interval: 15 Minutes . . . . . . . . . . . . . . . . 66

5.1.2 Smallest Time Interval: 30 Minutes . . . . . . . . . . . . . . . 68

5.2 Anaheim Network . . . . . . . . . . . . . . . . . . . . . . . . . 69

5.3 Chapter Summary . . . . . . . . . . . . . . . . . 73

6 Conclusions and Future Work $\quad 80$

$\begin{array}{ll}\text { References } & 82\end{array}$ 


\section{List of Figures}

1.1 Evacuation phases $[1] . \ldots \ldots \ldots \ldots \ldots$

2.1 Skeleton of four-stage traffic management function [2] . . . . . . . . . . . . 14

2.2 Optimization approaches in macroscopic evacuation planing [3]. . . . . . . 15

2.3 Static traffic assignment in simulation [4]. . . . . . . . . . . . . . . . . . . 17

2.4 Dynamic traffic assignment in simulation [4] . . . . . . . . . . . . . . . . . . . . 19

2.5 Decision making process for selecting traffic models [4]. . . . . . . . . . . . 21

2.6 Contra flow realized. . . . . . . . . . . . . . . . . . . 27

3.1 Static network structure before lane reversal. . . . . . . . . . . . . . . . . . 32

3.2 Static network structure after lane reversal. . . . . . . . . . . . . . . . 32

3.3 Discrete vs. continuous time horizon $[5] \ldots \ldots \ldots 33$

3.4 Time expanded network. . . . . . . . . . . . . . . . . . 34

3.5 Capacity measuring point. . . . . . . . . . . . . . . 36

3.6 Relationship between maximum flow and length of road segment. . . . . . 36

3.7 Effectiveness of vehicle positions on travel time [5] . . . . . . . . . . . 38

3.8 Travel time function $[5] . \ldots \ldots \ldots$. . . . . . . . . . . . . . . 40

3.9 Flow balance condition. . . . . . . . . . . . . . . . . . . . . . . . . . . . . . . . 42

3.10 Collision prevention constraint graphically. . . . . . . . . . . . . . . 43

3.11 Collision prevention with quickest regular flow travel time equal $2 \delta . \ldots \quad 45$

3.12 Collision prevention with quickest regular flow travel time equal $3 \delta$. . . . 45

4.12 node network. . . . . . . . . . . . . . . . . . 54

4.2 Solution for time expanded network with lane reversals. . . . . . . . . . 56

4.3 Solution for time expanded network without lane reversals. . . . . . . . . . 58

4.46 node network. . . . . . . . . . . . . . . . . . . . . . 59

5.1 Sioux Falls Network $[$ Yang and Meng]. . . . . . . . . . . . . . . . 67

5.2 Sioux Falls Network Objective Value vs. Demand, Smallest Time Interval: 15 Minutes. . . . . . . . . . . . . . . . . . . . 68

5.3 Sioux Falls Network Solving Time vs. Demand, Smallest Time Interval: 15 Minutes. . . . . . . . . . . . . . . . . . . . . . 69

5.4 Sioux Falls Network Objective Value vs. Demand, Smallest Time Interval: 15 Minutes. . . . . . . . . . . . . . . . . . . . . . . . 70 
5.5 Sioux Falls Network Solving Time vs. Demand, Smallest Time Interval: 15

Minutes. . . . . . . . . . . . . . . . . 71

5.6 Map of Anaheim. . . . . . . . . . . . . . . . . . . . . 72

5.7 Anaheim Network Objective Value vs. Demand. . . . . . . . . . . . . 73

5.8 Anaheim Network Solving Time vs. Demand. . . . . . . . . . . . . . . . 74

5.9 Partial and Complete 2-Directional O-D Pairs Objective Value vs. Demand. 75

5.10 Partial and Complete 2-Directional O-D Pairs Solving Time vs. Demand. . 75 


\section{List of Tables}

1.1 Man-Made Disasters . . . . . . . . . . . . . . . . . . . . 2

1.2 Natural Disasters . . . . . . . . . . . . . . . . . . . . . . 3

2.1 Different variables with respect to type of disaster [3] . . . . . . . . . . . 12

2.2 Four Stage Macroscopic Traffic Management Function [2]. . . . . . . . . . . 13

2.3 Instantaneous and Experience Travel Time Differences [4]. . . . . . . . . . 20

3.1 Indices and definitions. . . . . . . . . . . . . . . . . . . . 47

3.2 Decision variables and definitions. . . . . . . . . . . . . . . . . 48

3.3 Parameters and definitions. . . . . . . . . . . . . . . . . . . . 48

4.12 -Node Model, DTA-UB vs. NRDTA-UB. . . . . . . . . . . . . . . 59

4.26 -Node Model, DTA-UB vs. NRDTA-UB. . . . . . . . . . . . . . 64

5.1 Sioux Falls Network Results, Smallest Time Interval: 15 Minutes. . . . . . 76

5.2 Sioux Falls Network Results, Smallest Time Interval: 30 Minutes. . . . . . 77

5.3 Anaheim Network Results. . . . . . . . . . . . . . . . . . . . . 78

5.4 Anaheim Network Results with Complete 2-Directional O-D Pairs. . . . . . 79 


\section{Chapter 1}

\section{Introduction}

There are not many situations that call for a mass evacuation of residents in an area. Probably the most valid reason of them all is when a disaster strikes and evacuation is inevitable. However, evacuations are so rare that residents are unable to rely on experience alone to decide the time to take leave, the routes to select, and the destination to head towards for optimum result of the entire evacuation process. Thus, it is impossible for system equilibrium to emerge that distributes demand evenly across evacuation routes [6] or system optimum to be achieved that optimizes the total evacuation process. This chapter reviews examples of disaster that occurred in the past that called for an evacuation of local residents, recent work on emergency evacuation planning, and explains the importance of having a plan. The objective of this research also is stated together with an organization outline of this thesis at the end of this chapter.

\subsection{Man-made and Natural Disasters}

The world has been struck by disasters again and again throughout history, regardless of whether they are man-made or natural occurrences. It is an inevitable fact that disasters result in tremendous rebuilding cost for the authorities due to the massive destruction caused. In many cases, loss of lives is also an unfortunate consequence. Table 1.1 and 1.2 [7] are examples of past and recent man-made and natural disasters in the United States and around the world. Extensive studies done by Hooke [8] and Newkirk [9] show that 
the intensity and number of natural disasters have been increasing over the past decades. Dybas [10], in a survey also indicated that the duration and intensity of hurricanes have increased in the past three decades. As such, emergency evacuation planning is an issue of utmost importance in times of disaster in order to minimize the potential loss of lives.

Table 1.1: Man-Made Disasters

\begin{tabular}{|c|c|}
\hline Incident & Description \\
\hline Chernobyl Nuclear Disaster & 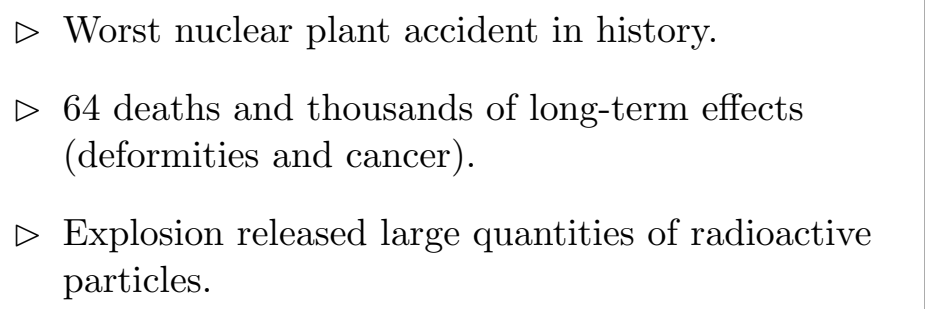 \\
\hline Fukushima Daiichi Nuclear Disaster & $\begin{array}{l}\triangleright \text { Triggered by earthquake and tsunami. } \\
\triangleright \text { Overheating of reactors causing potential explosion. } \\
\triangleright \text { Radiation detected all the way in West Coast of } \\
\text { United States. }\end{array}$ \\
\hline September 11 Terrorist Attacks & $\begin{array}{l}\triangleright \quad 4 \text { coordinated suicide attacks in New York } \\
\quad \text { and Washington DC. } \\
\triangleright \quad \text { Claimed lives of nearly } 3000 \text { people. } \\
\triangleright \quad \text { Islamist militant group, al-Qaeda, } \\
\quad \text { claimed responsibility. }\end{array}$ \\
\hline
\end{tabular}


Table 1.2: Natural Disasters

\begin{tabular}{|c|c|}
\hline Incident & Description \\
\hline Hurricane Katrina & $\begin{array}{l}\triangleright \text { Deadliest U.S. hurricane since } 1928 . \\
\triangleright \text { Claimed 1,833 lives at New Orleans, LA and surrounding area }\end{array}$ \\
\hline Indian Ocean Tsunami & $\begin{array}{l}\triangleright \text { Among the deadliest natural disaster in human history. } \\
\triangleright \text { Over } 230,000 \text { people killed in } 14 \text { countries in Asia. }\end{array}$ \\
\hline Haiti Earthquake & $\begin{array}{l}\triangleright 316,000 \text { people killed and } 300,000 \text { injured. } \\
\triangleright \text { Over } 1 \text { million people made homeless. }\end{array}$ \\
\hline Hurricane Sandy & $\begin{array}{l}\triangleright \text { Devastated portions of the Caribbean and Northeastern U.S.. } \\
\triangleright \text { Over } 250 \text { people killed in } 7 \text { countries. } \\
\triangleright \text { Second costliest Atlantic hurricane ( } \$ 65.6 \text { Billion). }\end{array}$ \\
\hline
\end{tabular}

\subsection{Emergency Evacuation Planning}

Emergency evacuation planning is the process of evacuating residents from hazardous zones to safe zones within the shortest time and using the most efficient way [11]. In the event of a disaster, an evacuation plan should be able to be implemented immediately. This will enable the authorities to make urgent decisions such as the allocation of evacuees, safe routes for evacuees to take, and locations safe from the immediate impact of the disaster to head towards [3]. Chen et al. [12] and Chiu et al. [13] describe evacuation planning in four critical operational decisions: (1) where to evacuate people (destination); (2) best routes to take (route); (3) flow rates regulation (traffic assignment); (4) rate at which evacuees are allowed to enter network (departure schedule). Stepanov et al. [1] dissected the process of evacuation planning into seven different phases as shown in Figure 1.1. In practice, an emergency evacuation plan needs to integrate all phases in order for it to provide efficient analytical results, optimal routing assignment, and to manage the evacuation process in real-time. The misallocation of highway capacities, inadequate communication with the public, and absence of logistic support during Hurri- 
cane Rita in September 2005 resulted in a chaotic evacuation of the residents of Houston [2]. This prompted a call for a well-planned and highly dynamic evacuation strategy to be implemented across United States, especially for large metropolitan areas.

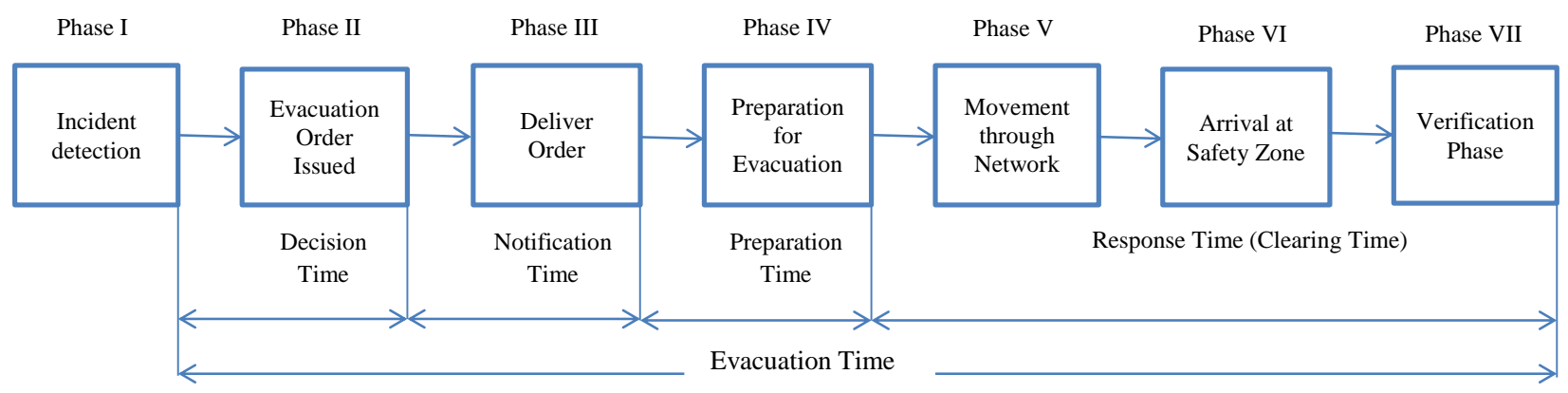

Figure 1.1: Evacuation phases [1].

\subsubsection{Related Work}

Much research has been done to make evacuation planning more efficient and realistic over the past thirty years or so. Early work primarily focused on nuclear power plant accidents and natural disasters. After September 11, 2001, more and more literature related to evacuation planning began to incorporate the potential threat of terrorist attacks and other man-made disasters when modeling evacuation scenarios mathematically. To date, a wide variety of material is available involving different analytical approaches to solve various mathematical models that strive to improve evacuation planning.

There are two broad aspects in evacuation planning: (1) moving people out of the disaster zone, and (2) moving people and material into the same area (i.e safe shelters and refuge areas) [14]. Kongsomsaksakul et al. [15] utilized a bi-level programming problem to model assignment of shelters within disaster zones for evacuees to escape to in times of disaster. Others proposed efficient algorithms to evacuate residents to safety zones, out of the reach of immediate danger $[13,1,2]$. Several methods exist that strive to optimize different aspects in evacuation planning, based on the kind of instructions that is being optimized. Stepanov et al. [1] seeks to optimize route assignment while Saadatseresht et al. [16] optimizes destination instructions. Liu [17] and Ben [18] on the other hand incorporated both route and destination instructions to optimize. Shen et al. 
[19] models a transportation network which responds to potential lane disruptions as a result of the disaster at hand to ensure safe, organized, and quick movement of people. In his review on travel behavior during evacuation, Pel et al. [20] focuses predicting of travelers' decisions regarding their choice to evacuate, departure time, destination choice, and route choice.

\subsubsection{Importance of an Emergency Evacuation Plan}

Ultimately, it has been proven that emergency evacuation planning that provides optimal instructions is essential in times of disaster. In a case study [21] done on Walcheron, Netherlands, Huibregtse concluded that the effectiveness of evacuating residents using an optimal evacuation plan more than doubled as opposed to residents using their own judgement in choosing nearest destination via shortest route. Due to the lack of information and experience in extreme situations, people act out of shear instinct to minimize his/her own travel time [21]. This results in the evacuation process not being optimal. With an optimized evacuation plan, people can act according to what is best for the system which in turn produces system-optimal condition [21].

\subsection{Research Objective}

As stated above, it is clear that even North America isn't spared from man-made or natural disasters in past times. Hurricane Irene, Hurricane Katrina, and Hurricane Sandy are recent examples of natural disasters. This research seeks to provide an effective tool and realistic tool for the authorities to further improve the current standards of emergency evacuation planning. To achieve this, a dynamic traffic assignment (DTA) technique is utilized to mathematically model an emergency evacuation plan that has the ability to tackle multiple sources and multiple destinations.

In situations where evacuation is needed, whether large or small scale, traffic comes to a standstill due to high demand of road usage. This results in tremendous delay in evacuating residents causing possible endangerment of lives. A solution is to have an effective system of evacuating residents in times of emergency through managing lanes. 
This research addresses the overall delay during evacuation and reduces it through traffic flow reversals of opposite lanes. In other words, whole segments of roads are to flip its usual directional flow to head towards the opposite direction in order to increase outbound capacity in an evacuation situation. In what usually is a two-way road, both lanes to be of one direction leading out of dangerous zone is considered. This holds great potential in the transportation sector, which when applied will result in tremendous cost savings for the authorities and ultimately, minimizing the potential loss of lives. Despite the tremendous advantage it brings to incorporate lane reversals, one must be cautious in doing so. The potential threat of head-on collisions of vehicles heading in two directions on the same road segment emerges as a result. To minimize the threat of such an outcome, a "Collision Prevention" constraint is added which restricts vehicles entering a particular segment and ensuring that it is cleared before its direction is reversed.

The original problem is formulated as a mixed integer nonlinear programming, system optimum DTA (DTA-SO) problem. The nonlinearity attribute makes the problem complex and tough to solve. Also, when applied to more general networks, the model becomes tremendously large and makes computation difficult. Through various linearization techniques and applied theorems, the problem becomes a mixed integer linear programming, upper bound DTA (DTA-UB). When solved, it provides computational results with very high precision. The steps to obtain a mixed integer linear programming DTA-UB and its formulation is extensively explained in the subsequent chapters and experimental results to prove the model's effectiveness is provided. The objective of this research is to minimize the objective function of travel time and late departure penalties and compare the improvement of results to that of a model without lane reversal.

\subsection{Thesis Organization}

This report is organized to help readers understand the background of the topic at hand and the motivation behind conducting this research. It is important to have these in mind first, in order to fully comprehend the purpose of formulating the problem in the way the author did. Chapter 2 breaks down emergency evacuation planning into different 
characteristics that relate to the model considered in this research. Recent literature related to the topic at hand are also reviewed and the chapter concludes by stating the significance of this project. Chapter 3 expounds on the model and gives the overall framework of the problem formulation, with each constraint that contributes to the model explicitly explained. An analysis of the model is conducted in Chapter 4 with the model without lane reversals presented. It also presents detailed computational results of small networks that can be understood with relative ease. Computational results of larger and more realistic networks are given in Chapter 5 and they are presented in both tables and graphical forms. Finally, Chapter 6 concludes this research with recommendations to be implemented in the future. 


\section{Chapter 2}

\section{Review of Emergency Evacuation Characteristics}

Two different classifications exist when considering emergency evacuation planning. Microscopic planning, as the name dictates, assesses minute aspects such as evacuee's behavior, movement, and interaction. Macroscopic planning, on the other hand, focuses on aspects involving the operation of evacuation as a whole such as the amount of people to be evacuated, capacity of roads, and number of danger or safety zones. Through much study on this topic, traffic assignment is shown to be the most popular and effective way to model emergency evacuation planning [2, 22, 20, 13, 12, 23]. Although known to have its own advantages, static traffic assignment does not provide a realistic representation of a real-life evacuation because changing evacuation conditions with time is not considered. Hence, a dynamic traffic assignment, although more complex, is used in this research to model a more realistic evacuation situation. Many techniques exist when seeking to obtain good computational results when solving dynamic traffic assignment for evacuation planning, but the two most commonly used are simulation approach and analytical approach. Many tools and softwares are available with regards to both approaches. The sections below further elaborates on microscopic and macroscopic planning, describes static and dynamic traffic assignment together with its advantages and disadvantages, and explain the differences between simulation based and analytical based approaches when solving dynamic traffic assignment. Literature involving the implementation of contra-flow is al- 
so reviewed below. This chapter concludes by reiterating the significance of this specific research.

\subsection{Microscopic Evacuation Planning}

Microscopic evacuation planning is mainly used for modeling individual entities' (people, vehicles, etc.) behaviors, movements, and interactions during the evacuation period [2]. Parameters that are usually considered for microscopic planning are traveling speed, reaction time, and interaction outcomes of each evacuee with other evacuees during evacuation. On top of having numerous factors to consider in microscopic planning, many of these factors vary drastically with time. Stepanov et al. [1] when modeling a multiobjective evacuation routing considered evacuees behavior in times of emergency. Evacuees behavior, as Stepanov described, is a complex phenomenon where there are those who don't leave the region after being warned, those who leave prior to evacuation notice being issued, and those who respond when an evacuation notice is issued. As such, the decision to evacuate depends on the following factors [24]:

1. Person's perception of danger.

2. Family and social status.

3. Existence of family/friends at destination points.

4. Type of disaster at hand.

Techniques used for evacuees modeling include queuing models, transition matrix models, and stochastic models. In all cases, pedestrian and evacuees dynamics obeys the equation of motion [25]:

$$
\frac{d r_{\alpha}(t)}{d t}=v_{\alpha}(t)
$$

where $\frac{d r_{\alpha}(t)}{d t}$ is the temporal change of location of pedestrian $\alpha$, and $v_{\alpha}(t)$ is the pedestrian velocity. Helbing et al. [25] further describes that if $f_{\alpha}(t)$ denotes the sum of social forces 
influencing pedestrian $\alpha$ and $\xi_{\alpha}(t)$ are individual fluctuations reflecting unsystematic behavioral variations, the velocity changes are given by the acceleration equation:

$$
\frac{d v_{\alpha}(t)}{d t}=f_{\alpha}(t)+\xi_{\alpha}
$$

When modeling relatively few evacuees during an evacuation, crowd dynamics are partially compared with the behavior of gases [25]. However, when a massive amount of evacuees takes on the characteristics of equations (2.1) and (2.2), crowd dynamics are strikingly analogous to the motion of fluids:

1. Evacuees are similar to streamlines of fluids.

2. Emergence of stagnant crowds appears like the formation of river beds.

These concepts are utilized by Klupfel et al. [26] to simulate the evacuation processes on passenger ships. To date, many researchers have made unrealistic assumptions regarding evacuees' behavior, specifically evacuees' departure responds. Many assumed all who are warned will evacuate, some assumed all registered vehicles will respond, while others assumed only one evacuating vehicle per household [27]. Lindell et al. [6] emphasized the importance of background data collection when determining evacuees' behavior when evacuating. Size and distribution of resident population is highlighted by Smith [28] in his book and he depicts the population changes over time by the following demographic balancing equations:

$$
P_{a}-P_{c}=B-D+I M-O M
$$

where $P_{a}=$ population size at some point in time after the census; $P_{c}=$ population size at time of census; $B=$ number of births; $D=$ number of deaths; $I M=$ number of immigrants; and $O M=$ number of emigrants. Other variables affecting population evacuation behavior include:

1. Number of persons per residential household [29].

2. Number of evacuating vehicles per residential household [30, 31, 32]. 
3. Size and distribution of transient population [33, 27].

4. Percentage of residents' warning compliance/spontaneous evacuation [33, 27, 34, 35].

5. Percentage of transients' warning compliance/spontaneous evacuation [36, 29].

Yuan and Han [37] conducted a study in the event of a nuclear power plant accident, where evacuees choose routes according to Most Desirable Destinations (MDD) in the traffic network. Hareesh et al. [38] presented the concept of crowd behavior during an emergency in a large building structure based on artificial life. Pel [20] in a review on travel behavior modeling in dynamic traffic simulation models for evacuations focused on how travelers' decisions are predicted via simulation regarding choice to evacuate, departure time choice, destination choice, and route choice.

Due to the vast amount of data, modeling details, and drastic variation of parameters, simulation approaches are commonly used in microscopic planning [2]. Among the many simulation models available for microscopic planning are NETVAC, MASSVAC, OREMS, and CEMPS [20].

\subsection{Macroscopic Evacuation Planning}

Macroscopic evacuation planning is mainly used to design strategic evacuation plans that do not account for individual behavior and interactions [2]. Macroscopic planning produces tight lower bounds on expected evacuation time, and tight upper bounds for number of people successfully evacuated. In turn, these bounds enable us to analyze and modify the transportation network configuration to accommodate evacuation needs [2]. Unlike microscopic planning, macroscopic planning focuses on large number of quantitative and qualitative variables that is involve in modeling the "big picture" of the evacuation process. Depending upon the type of disaster, different variables are used for modeling [3]. Table 2.1 [3] presents the different variables with respect to the type of disaster.

Macroscopic evacuation planning is broken down by Lahmar et al. [2] into four stages in his paper for hurricane evacuation. Each of these stages aims to minimize evacuation 
Table 2.1: Different variables with respect to type of disaster [3].

\begin{tabular}{|l|l|}
\hline \hline \multicolumn{1}{|c|}{ Disaster Type } & \multicolumn{1}{c|}{ Variables } \\
\hline Flood & Shelter, routes, traffic flow, demand between shelter and origin [15]. \\
\hline Earthquake & $\begin{array}{l}\text { District, suppliers, earthquake risk index, capacity of commodity, } \\
\text { durable commodity [39]. }\end{array}$ \\
\hline Nuclear Power Plant & $\begin{array}{l}\text { Path capacity, total number of evacuees, initial locations, } \\
\text { location of evacuation destination, network size, traveling time [11]. }\end{array}$ \\
\hline Homeland Security & $\begin{array}{l}\text { Logistics, route distance, travel time, evacuation time, } \\
\text { time window [2]. }\end{array}$ \\
\hline
\end{tabular}

time and maximize number of evacuees that produces feasible routings and evacuation schedules based on available time and road capacity. Table 2.2 [2] explains the functions of each stage. Figure 2.1 [2] shows the process flow of the four-stage traffic management function which produces feasible routings and evacuation schedules based on available time and road capacity [2].

Although dependent on the type of evacuation and disaster at hand, in general, macroscopic evacuation consists of more quantitative variables as oppose to microscopic planning. When it comes to formulation of the model, macroscopic planning also steers toward deterministic computations although there may be stochastic aspects such as demand/user generation in the model. Hence, a wide variety of optimization approaches exist in literature when it comes to solving macroscopic planning problems. Yusoff [3] conducted a survey on optimization approaches for macroscopic emergency evacuation planning. The different approaches are summarized in Figure $2.2[3]$ below.

Heuristic algorithms has been more commonly used compared to other approaches [3]. The main objectives of heuristics approaches usually involve minimizing evacuation egress time and minimizing computational cost of producing evacuation plan. Time aggregated graph has also been incorporated in heuristic algorithms to represent time varying attribute in network [3]. Lu et al. [11], Shekhar et al. [40], and George et al. [41] are among the many who did work using heuristics. Mathematical modeling usually involves formulating the problem as a linear programming, or non-linear programming model. Dijkstra algorithm, modified Bellaman-Ford, Branch and Bound, Greedy Flows, Benders Decomposition, Column Generation, and Lagrangian are among the many algorithms 
Table 2.2: Four Stage Macroscopic Traffic Management Function [2].

\begin{tabular}{|c|c|}
\hline Stages & Description \\
\hline I. Rough-Cut Capacity Plan (RCCP) & $\begin{array}{l}\text { - Assesses general feasibility of plan within } \\
\text { available time window. } \\
\text { - Provides guidelines on network to accommodate } \\
\text { evacuation needs. } \\
\text { - Identifies maximum throughput of people. } \\
\text { - Provides upper bound for throughput and lower } \\
\text { bound on minimum evacuation time. }\end{array}$ \\
\hline II. Detailed Capacity Plan (DCP) & $\begin{array}{l}\text { - Solves more detailed model. } \\
\text { - Provide macroscopic evacuation plan with } \\
\text { detailed routes and schedules. } \\
\text { - Compromise plan needed if infeasible. }\end{array}$ \\
\hline III. Restricted Evacuation Plan (REP) & $\begin{array}{l}\text { - Explores viability of back-up plans when RCCP } \\
\text { is infeasible. } \\
\text { - Identifies most vulnerable zones and assigns } \\
\text { geographic priorities. } \\
\text { - Provide lower bound on time necessary to } \\
\text { evacuate a danger zone. }\end{array}$ \\
\hline IV. Enforced Evacuation Plan (EEP) & $\begin{array}{l}\text { - Explores back-up plans when DCP is infeasible. } \\
\text { - Evaluates alternative strategies when evacuees } \\
\text { can not be routed to their preferred destinations. }\end{array}$ \\
\hline
\end{tabular}

used to improve solving time and reduce computational cost of mathematical modeling. Work done by Goldblatt et al. [42], Chiu et al. [13], and Andreas et al. [43] involves mathematical modeling for evacuation planing. Problems focusing on traffic management are usually more suited to use dynamic network flows [3]. Network flow breaks down the entire process of evacuation planning into systematic and organized structures with each section geared to optimize the different phases in the evacuation process. The fourstage traffic management function introduced by Lahmar [2] belongs to a class of network flow. Network flow structure also facilitates the evacuation planner in the preparation of 


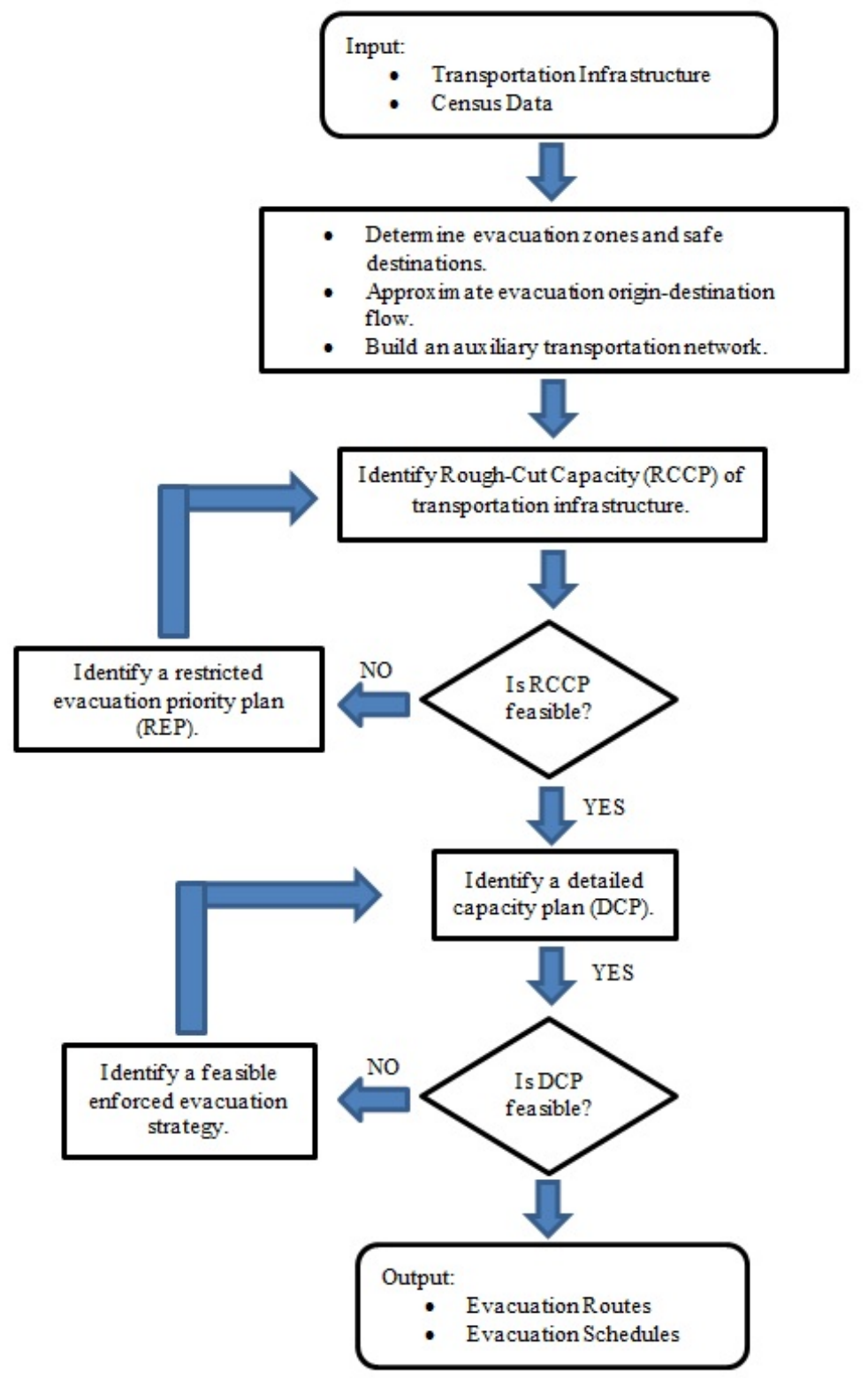

Figure 2.1: Skeleton of four-stage traffic management function [2].

evacuation plan according to zone vulnerability. Andreas et al. [43] and Kamiyama et al. [44] did work that utilizes network flow structure. Limited literature is available that deals with population optimization and exact method. Genetic algorithm (GA) was used by Liu et al. [17] to solve a two-level optimization model: 1. Minimize total clearance time. 2. Maximize throughput in targeted zones. Miller et al. [45] used SEscape as an exact method to support ERR for a fire disaster. SEscape functions to minimize total evacuation time, and maximize throughput capacity [45]. 


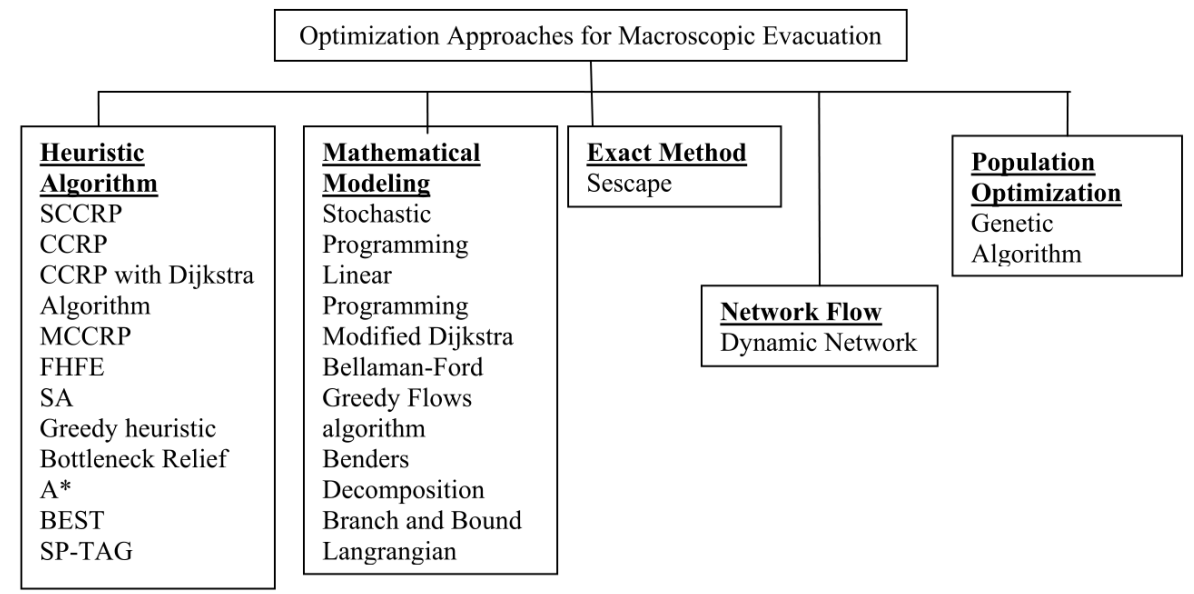

Figure 2.2: Optimization approaches in macroscopic evacuation planing [3].

\subsection{Static Traffic Assignment}

A traffic assignment model aims to estimate how traffic flows through a road system and the associated effects on the system [46]. Demand for travel, characteristics of transport system, and distribution of demand over the transport system are three kinds of information needed when solving traffic assignment models [46]. The demand for travel should be balanced across the entire transport system to achieve system equilibrium condition. In static traffic assignment models, congestion properties of each road are represented by volume-delay function (VDF) that is the average travel time on the road as a function of traffic volume [4]. Beckmann et al. [47] were the first to formulated the static traffic assignment as a mathematical model:

$$
\begin{aligned}
\operatorname{Min} z & =\sum_{a \in L} v_{a} c_{a}\left(v_{a}\right) \\
\text { s.t } \sum_{p \in P_{\text {od }}} e_{p} & =E_{\text {od }}, \quad \forall o d \\
v_{a} & =\sum_{o d} \sum_{p \in P_{o} d} e_{p} \delta_{p}^{a}, \quad \forall a \\
\sum_{p \in P_{\text {od }}} e_{p} & =E_{\text {od }}, \quad \forall o d \\
e_{p} & \geq 0, \quad \forall p \in P_{o d}
\end{aligned}
$$


Where $v_{a}$ represents flow of traffic on link $a, c_{a}$ is the travel time along link $a$ and is a function of link flow $v_{a}$. $E_{o d}$ represents traffic flow between origin $o$ to destination $d . \delta_{p}^{a}$ is an indicator variable:

$$
\delta_{p}^{a}= \begin{cases}1, & \text { if link } a \text { is on route } p \\ 0, & 0 \text { otherwise }\end{cases}
$$

By solving the model above, the static user equilibrium assignment problem is solved at the same time [47]. Karush-Kuhn-Tucker (KKT) necessary conditions [48] for minimum point can be used to prove the conditions of user equilibrium [46]. Many advanced algorithms written in books by Bazaraa [48] and Luenberger [49] can be used to solve the above system-optimum static traffic assignment model. In static traffic assignment, travel time correlates positively as throughput increases. Chiu et al. [4] highlights that because of the less complicated nature of static traffic assignments, mathematical properties can be obtained relatively easily. Additionally, a large advantage of static assignment is its low computational cost. In a relatively short time, static models can be optimized and approximate equilibrium solutions can be found.

\subsubsection{Static Traffic Assignment Limitations}

Despite the positive attributes of static traffic assignment, many limitations exist which outweigh its advantages. In his book, Patriksson [50] commented on assumption made on traffic assignment models:

"the fundamental principles underlying the assignment models were stated some forty years ago. The traffic flows in the then relatively uncongested urban networks were probably suitable for approximation by steady-state flows, as Wardrop did. Since those days, the traffic networks have become much more complex and the demand for transportation has become orders of magnitude higher, and the approximation of present traffic flows by steady-state flows is far less realistic."

Static assignment does not consider the potential increase of travel time due to increase in congestion and has no direct correlation with physical measures such as speed, density ,or queue, resulting in an unrealistic representation of real-life scenarios [4]. Without 
factoring in potential congestion, static assignment also does not consider flow-spill back. This causes the volume of roads to increase indefinitely and eventually exceeding the physical capacity of roads [4]. Figure 2.3 [4] shows the simulation structure of static

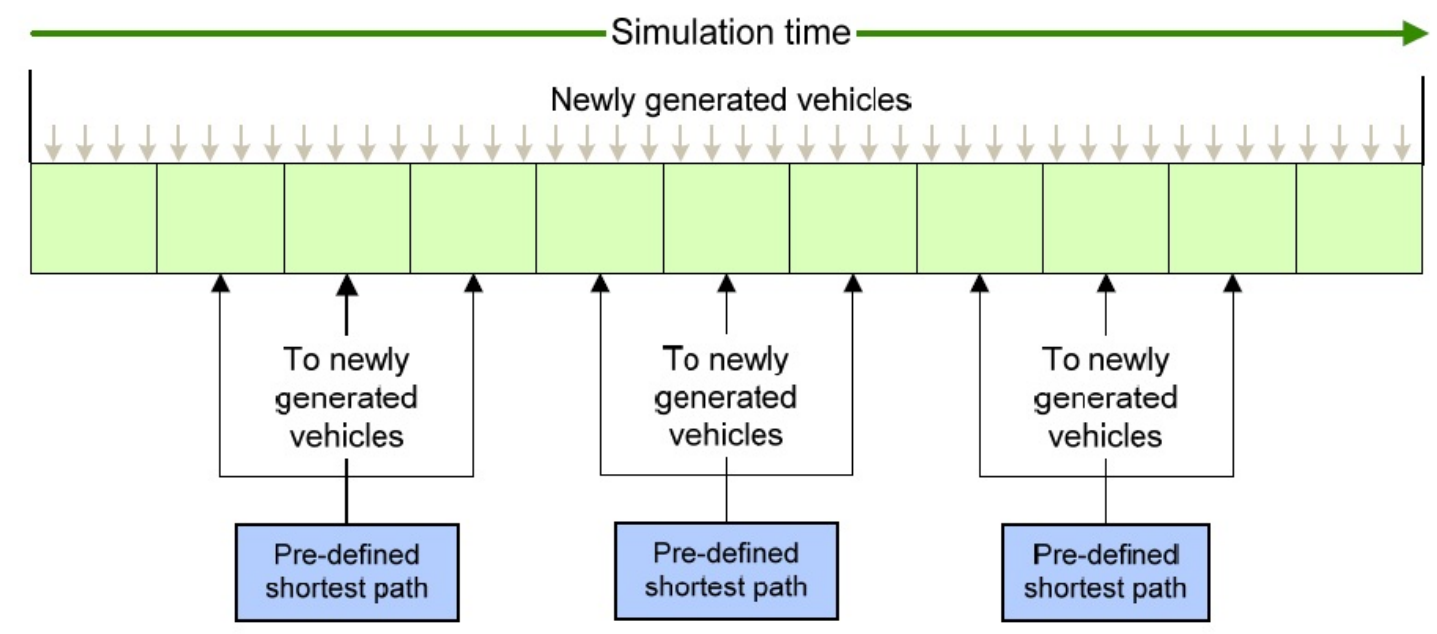

Figure 2.3: Static traffic assignment in simulation [4].

traffic assignment. In general, static approaches cannot reflect traffic variations over time and fail to consider the many measures taken to address congestion [4]. Hence, they are only effective in cases where mandatory evacuation with imposed route controls are implemented.

\subsection{Dynamic Traffic Assignment}

While static traffic assignment implies that vehicles stay on a specific route and also leave and arrive at a fix time period, dynamic traffic assignment (DTA) on the other hand allows users to choose direction at every road crossing and also grants them flexibility to depart accordingly. That being said, DTA is equivalent to expanding static assignment with respect to time where a duplicate of static network is considered for each discrete time period [2]. Although dynamic models have been around for a while, it is only during the emergence of intelligent transport system (ITS) due to technological advances that DTA started to be widely acknowledged [46]. Chiu [13] boils down the aim of DTA research in to main objectives: 1 . to support real-time route guidance and traffic management 
under ITS architecture; and 2. to improve operational planning practice that cannot be addressed by static traffic assignment. Based on Wardrop's principles [51], two approaches exist when dealing with DTA [46]: dynamic user equilibrium and dynamic system optimal assignments.

Formulations of dynamic user equilibrium can be grouped into five categories [46]:

1. Mathematical programming

2. Optimal control theory

3. Non-linear complementary problem

4. Fixed point problem

5. Variational inequality

The pioneer of dynamic user equilibrium was Friesz et al. [52] who formulated the problem using variational inequalities. Patriksson [50] showed that variational inequalities can be regarded as a generalization of mathematical programming, non-linear complementary problem, and fixed point problem. Dynamic user equilibrium represents the distribution of traffic based on users own interest, which results in inefficient use of the transport system [46].

Dynamic system optimal assignments on the other hand aim to calculate optimum time path for the decision variables such that the entire traffic flow achieves system optimal condition [46]. Merchant and Nemhauser [53] were the very first to come up with dynamic system optimal assignment model before others modified it to suit different scenarios.

DTA fuses multiple sources of information, estimate and predict evolution of network wide traffic conditions, and generate route guidance based on its ability to integrate historical data and information from multiple sensor sources [54]. Detailed algorithms within a DTA model further enable it to incorporate complex demand-supply interactions. DTA's ability to combine individual models into a complex system with many inputs and parameters makes it ideal for evacuation planning and management [54]. The computational efficiency of DTA models represents a powerful trade-off between modeling accuracy and 
running time. Different from static assignment, the simulation structure of DTA shown in Figure 2.4 [4] presents DTA's ability to sense, update, and re-optimize according to updated traffic conditions with respect to time. A variety of dynamic network flows has

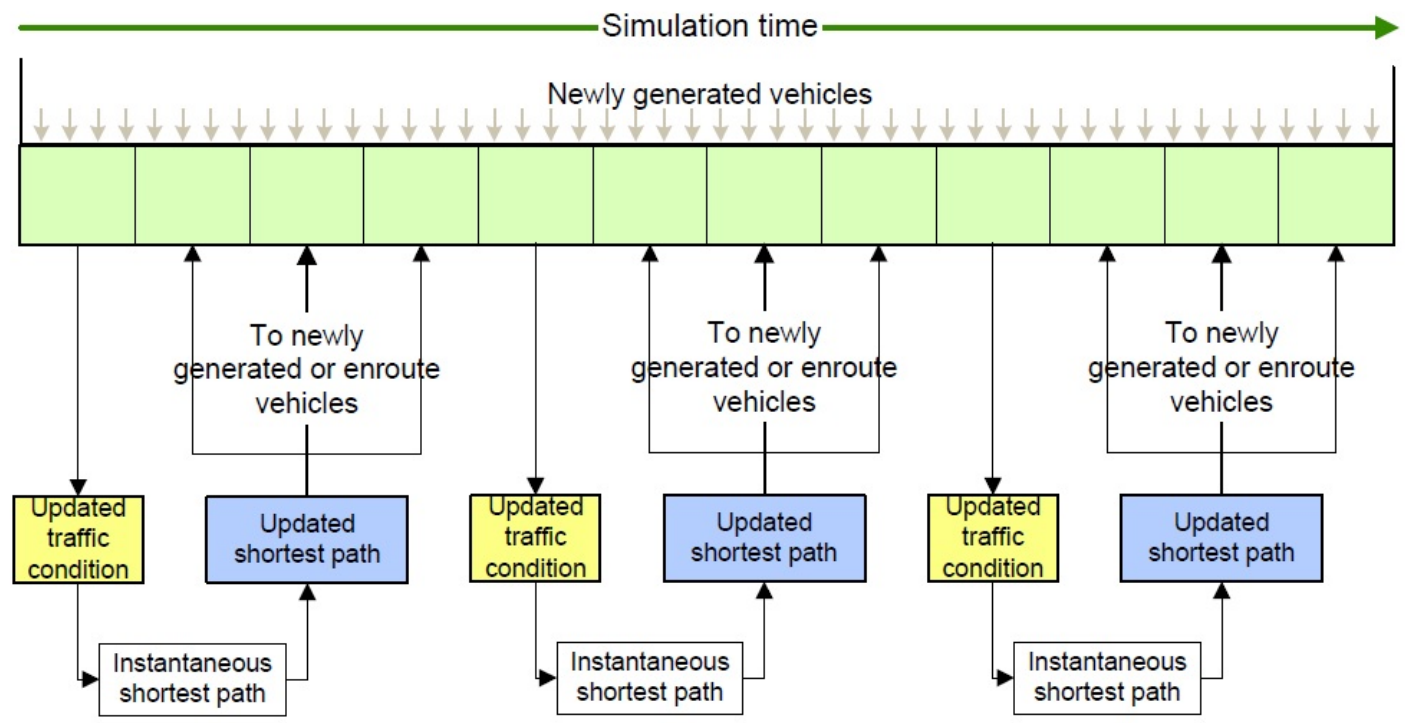

Figure 2.4: Dynamic traffic assignment in simulation [4].

emerged which seeks to optimize different aspects of evacuation planning. Maximum dynamic flow seeks to maximize amount of flow from origin to destination within a given time period [55]. Quickest dynamic flow seeks to minimize necessary time to send flow from origin to destination [56]. The universal maximum dynamic flow seeks to maximize amount of flow arriving at sink at all times before a specific deadline [57, 58, 59].

\subsection{Dynamic Traffic Assignment for Emergency E- vacuation Planning}

A common behavioral assumption in DTA is that users will select routes that has the shortest travel time. In reality, road users' definition of best route choice varies for each individual where lowest cost or minimum disutility are often considered as well [4]. As evacuating in the shortest time possible is the logical approach when it comes to evacuation planning, within limits, shortest travel time is always taken as the objective of the model and takes precedence over other factors. When an evacuee has been assigned 
such a route, every used origin and destination (O-D) pair has its own minimum travel time. This condition is known as user equilibrium [4]. The minimum travel time of each O-D pair is usually at the very least equal to the specific link's free flow travel time. Evacuees will not be able to find another O-D route that will further reduce travel time at user equilibrium condition as long as parameters such as network size or travel demand does not change. The notion of user equilibrium is extended in two ways when modeling DTA in a general sense [4]. The first extension assumes that road users have information regarding future road conditions and in choosing and O-D route, they are minimizing travel time they will experience. The second extension assumes that user equilibrium condition of equal travel times applies only to users that departs at the same time on the same O-D pair. DTA approach also typically uses experienced travel time as measurement as oppose to instantaneous travel time. The main differences between the two measurements are listed in Table 2.3 [4]. Since equilibrium assumes that users are well informed and make rational choices [4], when applied to emergency evacuation planning, it is natural that equilibrium condition is often not realized. Hence, evacuation planning research often focuses on disequilibrium models where evacuees do not have perfect information about network travel conditions. This is because of drastic changes of road conditions when disaster strikes resulting in minimum travel time routes differing from that of evacuees prior knowledge or experience.

Table 2.3: Instantaneous and Experience Travel Time Differences [4].

\begin{tabular}{|c|c|}
\hline Instantaneous Travel Time & Experience Travel Time \\
\hline $\begin{array}{l}\text { - Minimum travel time is based on snapshot } \\
\text { of the link travel times prevailing } \\
\text { at departure. } \\
\text { - Assumes that users know what the } \\
\text { shortest route is at departure. } \\
\text { - Does not necessarily result in minimal } \\
\text { experienced travel time due to } \\
\text { dynamically changing link travel times. } \\
\text { - No provision to reflect anticipated } \\
\text { congestion that is to occur at later time. }\end{array}$ & $\begin{array}{l}\text { - Yield time-dependent shortest route with } \\
\text { minimal experience travel time. } \\
\text { - Assumes users are willing to seek routes } \\
\text { that minimizes their experienced travel } \\
\text { time, instead of route that appears } \\
\text { best only at departure. } \\
\text { - Accounts for time needed for traveling } \\
\text { through an O-D link based on time of } \\
\text { entering. }\end{array}$ \\
\hline
\end{tabular}


A tremendous amount of factors come into play when selecting the right model to solve traffic problems. Among them are geographical location, situational condition, population size, and many more. For emergency evacuation planning, disaster type, road capacities, and travel mode are common factors to be taken into consideration. Often, selecting the right model not only depends upon information and facts available, equally importantly, experience is a factor that has a strong hold to enhance and improve modeling experience for the user. Technical report written by Jeannotte et al. [60] contains detailed methodology to select traffic decision support models. Chiu et al. compressed the steps for selecting traffic analysis tools into seven decision-making guidelines shown in Figure 2.5 [4]. The yellow shaded areas indicate attributes or capabilities generally supported by DTA models. The blue shaded areas are those generally considered in emergency evacuation planning.
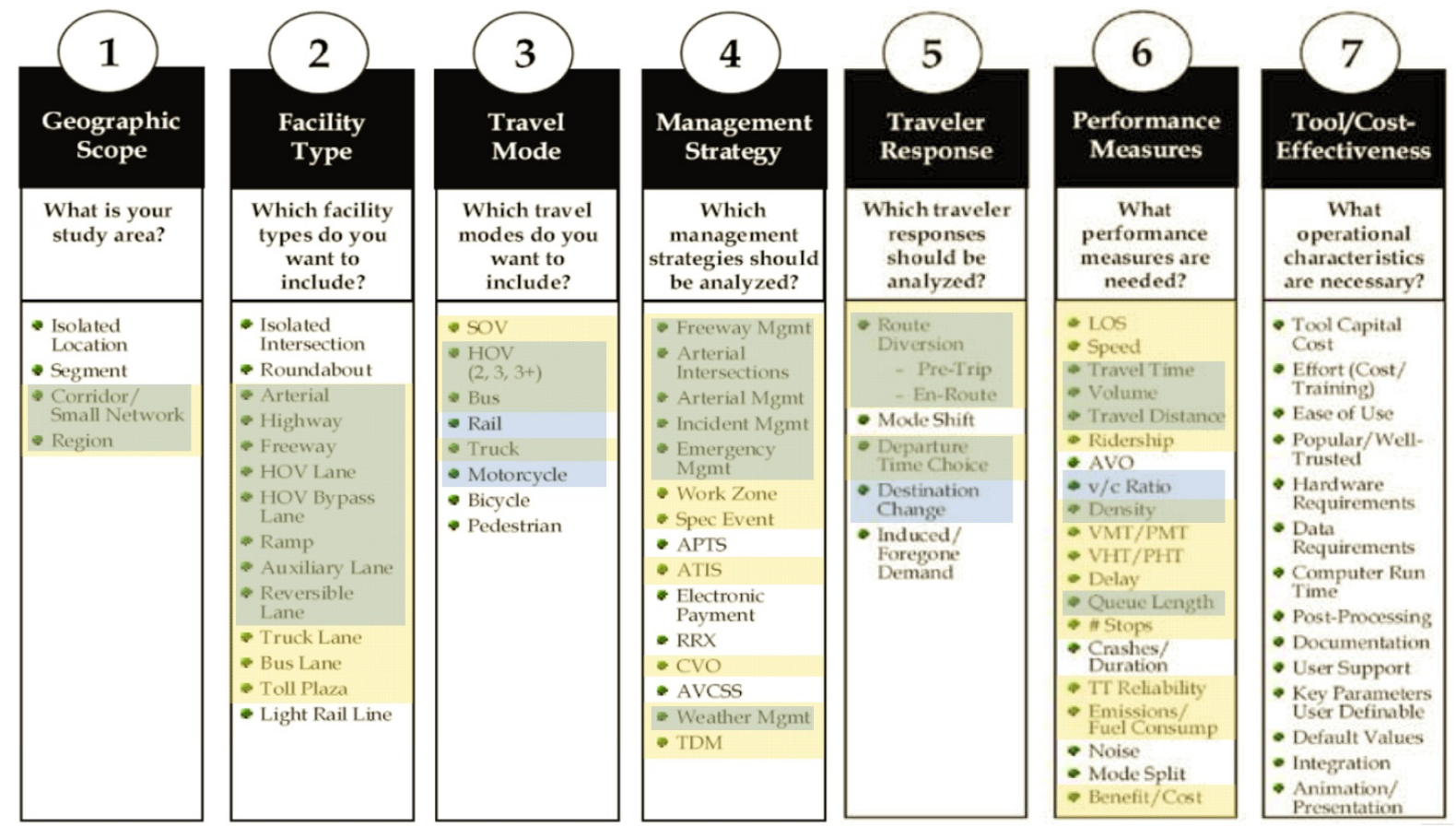

Figure 2.5: Decision making process for selecting traffic models [4].

Before results are able to be generated, inputs and parameters need to be specified. Often, these inputs and parameters will require calibration to depict a realistic and acceptable model depending upon the individual components that make up the DTA model. In general, they can be grouped into demand-side and network-side quantities [4]. 
Demand-side quantities:

- Trip tables

- Evacuees' behavior model inputs

- Demand at each specific node

Network-side quantities:

- Link capacities

- Link performance functions

- Planning time horizon

- Shortest time interval

- Number of safe and danger zones

Verifying results generated are crucial when validating one's model. Many different qualitative and quantitative analysis are used in this process. Some model users that are more vigorous will resort to using statistical tests, such as linear regression analysis to quantify the validity of the results. A rule of thumb for DTAs is that data used for validation must be dynamic in which the finer the time resolution of the date, the better the model represents real-world problem. In general, users should start by generating results for small networks. This enables them to detect discrepancies or inconsistencies before moving on to much larger and more realistic network sizes. Chiu et al. [4] list the characteristics of a DTA solution as follows:

- Vehicle departing at different times are assigned with different routes.

- Vehicles departing at the same departure time between the same O-D pair but taking different routes should have the same experienced travel time.

- Experienced travel time cannot be realized at departure, but only at the end of trip. 
DTA models offer the ability to make operational planning decisions such as making demand management actions or making planning decisions [4]. As such, DTA is the most ideal approach when dealing with emergency evacuation planning. Moreover, DTA is able to accommodate to temporal or spatial pattern shift of traffic which is a common phenomenon in evacuation planning due to sudden congestions of roadways. DTA for evacuation planning is generally classified into 2 different approaches: 1 . Simulation based and 2. Analytical approach. The sub-sections below elaborate on the main differences, advantages, disadvantages, and recent work done utilizing these two approaches. Extensive information regarding the usage of DTA such as selecting specific models, planning for DTA activities, data set preparation, and model calibration can be found in the primer, "Dynamic Traffic Assignment", by Chiu et al. [4].

\subsubsection{Simulation Approach}

Chiu et al. [13] describes simulation-based DTA as a mathematical programming based model in which vehicular traffic dynamics and the link/path travel time are estimated through a trial and error method. Simulation-based approaches are able to incorporate large amount of parameters and also accommodate to drastic changes of these parameters over time. Its ability to capture a tremendous amount of modeling details such as traffic flow dynamics, behaviorial characteristics of individual evacuees, and entities' interaction, makes simulation the ideal approach when dealing with microscopic evacuation planning [22], although at times it is also used in macroscopic and mesoscopic planning. Microscopic model simulations are able to analyze various geometric design configurations, evaluate localized individual intersections, and analyze interactions of multiple modes of transportation [4]. Simulation in macroscopic and mesoscopic scale, on the other hand, usually takes place on a section-by-section basis rather than tracking individual entities and their characteristics. They also operate on the basis of aggregate speed/volume and demand/capacity relationships [60]. Especially with small networks, simulation is able to provide relatively good and improved results in a short time, although optimality is not always guaranteed. Simulation is able to discover the path of vehicles on a mesoscopic 
scale for every O-D pair at every departure time interval. In turn, information on changes in traffic flow, temporal and spatial dynamics of travel times and other congestion indices can be extracted [4]. The attribute of dividing analytical period into time intervals enables simulation to evaluate buildup, dissipation, and duration of traffic congestion [60].

Despite its various advantages, the simulation-based approach has its own limitations. Its applicability, in most cases, is typically limited to function as a "what if" method to evaluate small sets of pre-specified candidate plans [22]. Due to it's time-consuming process when simulating large-scale networks even with the utilization of an advance computing system, simulation limits the ability to extensively search for optimality for realistic sized networks. As such, Xie et al. [22] describes simulation as only being able to be used for evaluating and assessing, but not for generating evacuation planning scenarios. Moreover, simulation approaches are unable to predict changes in design or changes in road environment which might affect potential collisions and driver behaviors [60].

NETVAC, IDYNEV, MASSVAC, SNEM, TEVACS, VISSIM, OREMS, CEMPS, and DynaMIT are among the few simulation softwares that are used for evacuation planning purposes [22].

\section{Applications of Simulation Approach}

Most literature available involving DTA commonly utilizes simulation when solving. Balakrishna et al. [54] used DynaMIT, a simulation system design, to combine real-time data from surveillance system with historical data to estimate current network state, predict future traffic conditions, and provide travel information through ATISs. DynaMIT was used in a case-study to ascertained network management strategies' benefit. Stepanov et al. [1] embeded $M / G / c / c$ queuing model in an integer programming model to capture time delay functions on road links for stochastic evacuation planning optimal route assignment. MGCCSimul simulation software was used to evaluate clearance time, travel distance, and congestion level. The model considers a Poisson arrival rate of vehicles $\lambda$, general service rate $G$, with a limited capacity $c$. Shen et al. [19] developed a bilevel model with upper level design to locate safety shelters and lower level to calculate user equilibrium travel times. A simulation algorithm, the Franke-Wolfe algorithm, was used 
to solve the lower level model. Han and Yuan [61] coded a most desirable destination (MDD) evacuation road network in VISSIM to simulate traffic evacuation operations. A case study on Tennessee's Sequoyah nuclear plant using this method showed results that has a $58 \%$ reduction of evacuation time as oppose to static destination selection and traffic assignment. A vast array of papers are available addressing different issues utilizing various simulation tools for various models. On the microscopic level, an extensive review on different dynamic simulation models for evacuation can be found in the article by Pel et al. [62].

\subsubsection{Analytical Approach}

Different from simulation-based approach, Chen et al. [12] describes analytical-based models consisting of mathematical programming or variational inequality problems in which the link travel times are estimated through closed-form path performance function and deterministic procedures. Often times, analytical approaches for evacuation planning can be directly used to search for optimal evacuation solutions. The model user defines and inputs data and parameters through a vigorous calibration process to depict an as close to real-life as possible situation and produce a single optimal solution. Typically, the analytical approach leads to an evacuation planning model written in a mathematical programming or other optimization-based functional forms, whose objective is set as minimizing the total evacuation time, minimizing the network clearance time, or maximizing the network traffic throughput [22]. Unless there exist stochastic attributes within the model, analytical approaches will consistently yield the same optimal solution given the same set of inputs and parameters. As such, analytical tools are able to quickly predict capacity, density, speed, delay, and queuing on a variety of transportation networks [60]. As oppose to simulation-based approaches, analytical approaches are able to tell the user "what to do" on making an evacuation plan [22]. They are also quick, reliable, and tested through significant field-validation efforts [60].

A limitation of analytical approaches is that they assumes conditions of one road segment not affecting conditions of adjacent roadways [60]. In reality, heavy congestion and 
long queues on one segment usually interferes with other locations. Even though most reasonably sized networks are capable of being solved using analytical approaches, at times large sized data will result in situations where the model is unsolvable. Depending on the formulation of the mathematical model, computational results may come back infeasible if insufficient planning time horizons are assigned, or the operating system used to solve the model may run out of memory as a result. To overcome this limitation, many algorithms have been developed over the years to simplify/decompose models, reduce computational time, and obtain optimal solution for tremendously large networks. Some of these algorithms include Modified Dijkstra algorithm, Benders Decomposition, Branch and Bound method, and Column Generation algorithm [3]. Detailed description of advantages and limitations of analytical approaches are found in a traffic analysis toolbox by Jeannotte et al. [60].

\section{Applications of Analytical Approach}

Xie et al. [22] developed a bi-level optimization model where the upper level optimizes network evacuation performance and lower level simulates dynamic evacuation flows. Lagrangian-relaxed algorithm was used to reduce structure complexity and provided a algorithmic framework to seek optimal solutions. In another paper [63], Xie et al. developed a method to reconfigure existing networks to identify specific route to be reserved for emergency vehicles to access evacuation areas. Another bi-level optimization system was presented by Liu et al. [64] where upper level maximizes throughput during evacuation and lower level minimizes total travel time. For large-scale network applications, a revised cell-transmission formulation was imposed to efficiently model flow propagation and yield reliable performance. Yao et al. [65] developed a robust linear programming model based on robust optimization approach. Significantly less literature can be found that implements different analytical techniques for evacuation planning compared to that of simulation approaches. Nevertheless, all the above mentioned papers concluded that sound improvements and advantages were found with the implementation of analytical techniques. 


\subsection{Contra-Flow / Lane Reversals}

The majority of freeways, highways, arterial road, or collector roads here in United States are two-directional, where a road user heading in a certain direction will often encounter traffic flow heading in the opposite direction on the other side of the roadway. Contra-flow or lane reversal is realized when a road segment is reversed to head toward the opposite direction from it's regular flow direction. Figure 2.6 shows a road segment where a lane is reversed.

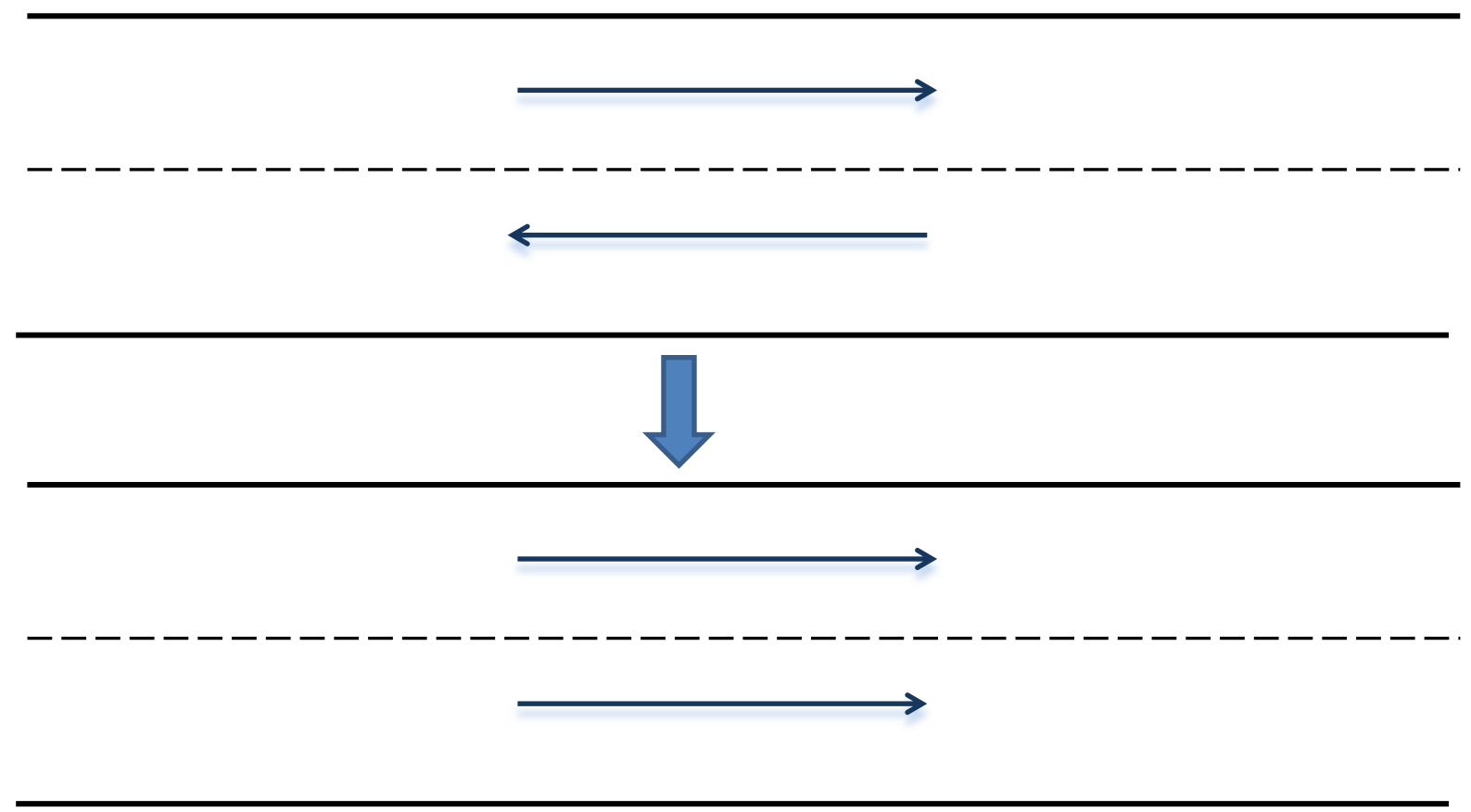

Figure 2.6: Contra flow realized.

Although lane reversals have been implemented in different events such as capacity control during off-work peak hours and football games [40], it's application is most crucial in the event of a disaster that calls for evacuation of residents. Heavy congestion and stand-still traffic is a common phenomenon in times of emergency as residents eagerly seek to evacuate for safe refuge. Hence, lane reversal is a remedy to tremendously increased outbound capacity and help ease road congestion. Having an increase of outbound capacity naturally also leads to an increase in residents evacuated and shorter evacuation time. Often times, a road segment need not be two-directional for lane reversal to occur. 
A one-directional road segment can also be reversed to flow in the opposite direction as the the main objective is to increase outbound capacity. Contra-flow/lane reversals is not a new concept by any means. Eleven coastal states facing constant potential threats by hurricane disasters have considered implementing lane reversals in their evacuation strategy [66]. Actual usage of lane reversals are also seen during Hurricane Floyd in North and South Carolina, and also during Hurricane Georges in Louisiana and Mississippi [66]. Despite definite plans and occasional implementation of lane reversals, a survey done by Wolshon, Urbina, and Levitan [67] reveals that the authorities and planners have no recognized standards or guidelines for the design, operation, and location of lane reversal segments.

Literature involving contraflow/lane reversal include a work done by Shekhar and Kim [40] where heuristics is used to tackle multiple sources and multiple destinations network for evacuation planning. Flip High Flow Edge (FHFE) heuristic was used to prioritize edges in order to reverse them. Simulated Annealing (SA) was also used to to escape local minima situations in an iterative way. Xie et al. [22] incorporated lane reversals and crossing elimination strategies to increase capacity in specific directions through the evacuation network. Hamza et al. [68] introduced multicast routing problem and breadthfirst graph traversal, two contra flow algorithms, to handle single-coordinated incident. Other papers on contraflow and lane reversal [66, 67, 69, 70] mainly tackle managerial and operational aspects of contraflow. Planners and decision makers often rely on past experiences and guesses to design lane reversals for evacuation planning which is not ideal when dealing with constantly changing demographic data and road layouts [40].

\subsection{Project Significance}

The goal of this project is to provide a model that is computationally efficient and able to depict a realistic representation of an emergency evacuation situation. As oppose to having to consider users behavioral characteristics, this thesis focuses on optimizing the process of evacuation planning as a whole where users' are assumed to choose routes and departure time to achieve optimality. Hence, a macroscopic approach is used to calculate 
the best mobility of evacuees during an evacuation situation. Although many quantitative and qualitative factors determine the definition of best mobility, it is safe to conclude that often times, the primary concern during an emergency evacuation is to minimize total delay/travel time of evacuees. Hence, best mobility is defined as dynamic traffic system optimum (SO), which denotes minimum total delay. In reality, travel demand between O-D pairs, users' choice of departure time, and total duration of entire evacuation planning process are time dependent. As such, dynamic traffic assignment (DTA) is the best technique to model evacuation planning as it offers the ability to make operational planning decisions with respect to time.

The mathematical model in this project is formulated as a mixed integer programming with discretized time horizon and utilizes link performance function to capture relationship of user travel time and travel volume on links in the network. A fair assumption is made where link travel time is directly affected by all vehicles present on the link. Intuitively, closer vehicles will affect traveling speed of a specific user more that those further away. Merchant and Nemhauser (1978) [53] utilized discrete time horizons when modeling DTA problem. Zheng and Arulselvan (2011) [5] incorporated link performance function for dynamic assignment for managed land system. Simultaneous route and departure (SRD) assumption was also kept in their model formulation where users can choose the route as well as departure time. Attributes of the models in these two papers are also contained within the model presented in this thesis.

The main contribution of this thesis is the ability to have potential contraflow/lane reversal when necessary to increase outbound capacity. Increase of outbound capacity will in turn result in increase of evacuees successfully evacuated and also decrease of total travel time. With the realization of lane reversals, the threat of potential head on collisions with oncoming traffic naturally emerges. To prevent such an occurrence, directional flow needs to be strictly restricted where all vehicles on a link cleared is ensured before it's flow direction are reversed and vehicles heading towards the opposite direction is allowed through. In order to validate the effectiveness of the proposed model, computational results are recorded and compared with a model without reversals. To our knowledge, there hasn't been work done on DTA for evacuation planning that incorporates lane 
reversals for the entire network that tackles multiple sources and multiple destinations, and incorporates collision avoidance on links and solving it as a system optimal model. As such, it is desired to be able to contribute an advance, efficient, and practical way for the authorities to further improve current standards of evacuation planning through this research. Detailed description of the formulation of this model is presented in the next chapter. 


\section{Chapter 3}

\section{Problem Formulation}

The problem formulation in this chapter is an expansion of the model concept used by Zheng and Arulselvan [5]. In their paper, a discrete time DTA model was used for traffic system with managed lanes. Their objective is to predict traffic flows on managed lanes at various times in the entire planning horizon and strive to minimize overall delay. Zheng and Arulselvan's model formulation concept is found to be directly applicable in the context of evacuation planning with appropriate modifications. Instead of modeling the problem as having restricted and unrestricted lanes in a managed lane traffic system, in an evacuation system, all lanes are treated as regular (general-purpose) lanes able to be used by everyone and able to be reversed when needed. Figure 3.1 shows a static network structure of a two-directional traffic system where there are three points users are able to either enter or leave the road. Each of these points are represented by a node and the road segment between two adjacent points is represented by arcs. In this case, there are two arcs connecting every pair of adjacent nodes as there are two lanes in this entire road segment. The two parallel arcs usually have different capacities and link performance functions. Figure 3.2 shows the situation where a lane is reversed to increased the outbound capacity away from the disaster zone. Time expanded network structure is introduced in the next section. After that, the formulation of every constraint and its purpose is explained. The full model is presented subsequently with detailed descriptions of all indices, variables and parameters. We end this chapter by stating the various assumptions made in the model and its limitations. 


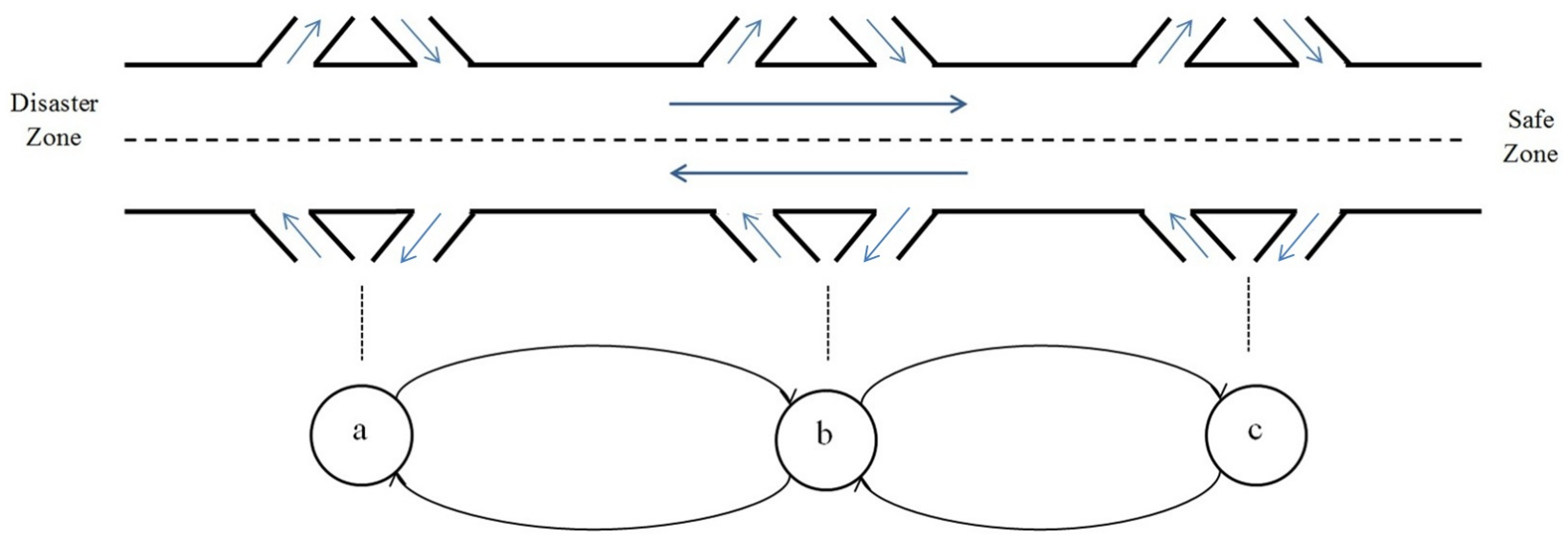

Figure 3.1: Static network structure before lane reversal.

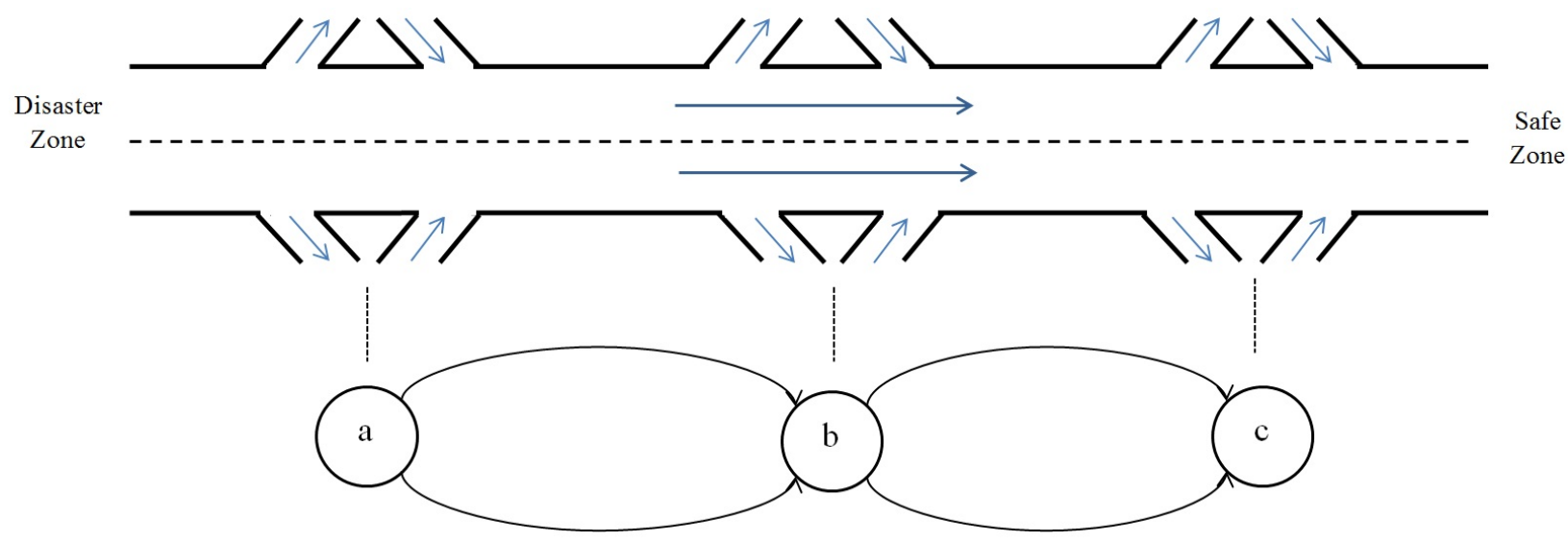

Figure 3.2: Static network structure after lane reversal.

Note: Sections 3.1 and 3.3 are based on the paper "Discrete time dynamic traffic assignment models and solution algorithm for managed lanes" by Zheng and Arulselvan [5]. Some modifications, however, have been made for it to be applied in the context of evacuation planning.

\subsection{Time Expanded Network}

Zheng and Arulselvan [5] makes use of the time expanded network and a DTA formulation based on the discretization technique employed in the paper by Nahapetyan and Lawphongpanich [71]. The continuous planning time horizon is $[0, T]$ is discretized into a set of discrete time points $\Gamma=\{0, \delta, 2 \delta, 3 \delta, \ldots, C \delta\}$, where $T=C \delta$ is assumed. Figure 
3.3 illustrates this concept. Here, $\delta$ represents the subdivision of the planning horizon, which is also the smallest time period in the formulation. For example, let $T=10$ and take $\delta$ to be 2 , and then $C=5$.

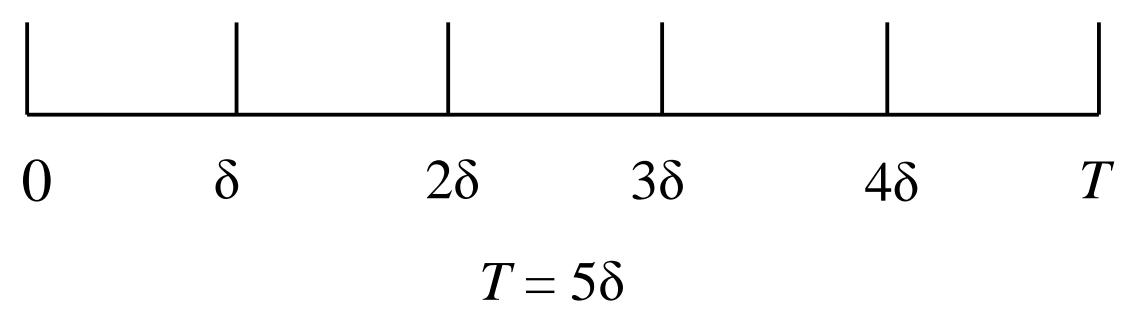

Figure 3.3: Discrete vs. continuous time horizon [5].

The time expanded network is obtained by first replicating each static node $C+1$ times. For example, a static node $p$ is replaced by the time expanded nodes $p_{0}, p_{\delta}, p_{2 \delta}, p_{3 \delta}, \ldots, p_{C \delta}$, where the subscripts denote the discrete time points. Next, time expanded arcs will take the place of the original set of static arcs, $A$. Travel time of each arc is also discretized by $\delta . \Delta_{p, q}$ is used to denote the set of all possible discrete travel times for arc $(p, q)$. A time expanded arc is denoted by both head and tail nodes (time expanded nodes) in time expanded network. The time expanded arc $(p, q ; t, t+\tau)$, where $\tau$ belongs to $\Delta_{p, q}$, connects time expanded nodes, $p_{t}$ and $q_{t+\tau}$. Each time expanded arc denotes a realization of travel time for the corresponding static arc. In this example, a time expanded arc $(p, q ; 1,3)$ would indicate the realization of the static arc $(p, q)$ with travel time $\tau=2$ (travel time is also being discretized), that connects node $p$ at time instance 1 and node $q$ at time instance 3 of our planning horizon $[0,10]$. In other words, the departure time is $t=1$ and arrival time $t+\tau=3$. An example of the time expanded network for the aforementioned static network is presented in Figure 3.4. In reality, a user departing at a certain time point will only be able to arrive at a later time point, which is why the dotted red lines in Figure 3.4 represents prohibited travel for road users. In this example, users departing at time period $\delta$ will only be able to arrive at time periods $>\delta$. The assignment of minimum and maximum travel time of each and every arc is dictated by the travel time function explained in Section 3.2. 


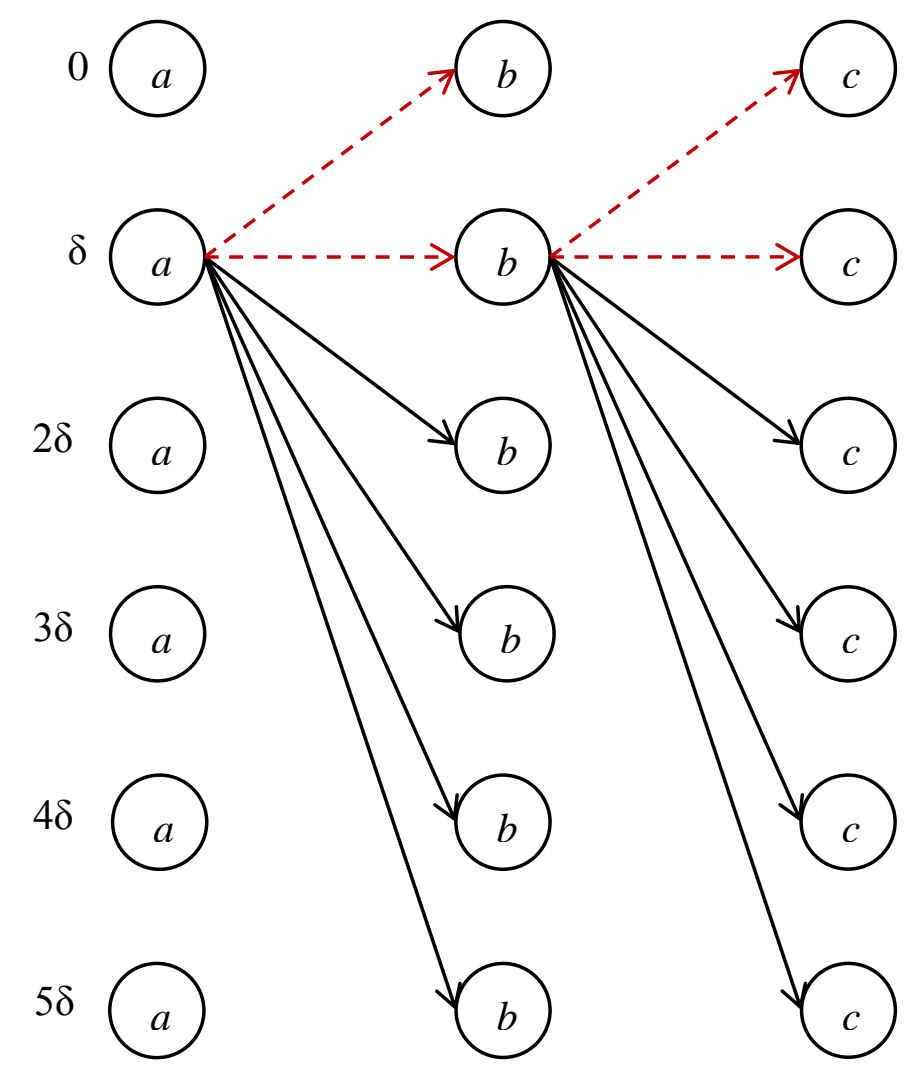

Figure 3.4: Time expanded network.

\subsection{Travel Time Function}

Since travel time on any static arc $(p, q)$ is time dependent, $\phi_{t}^{p, q}$ is used to denote travel time on $\operatorname{arc}(p, q)$ when a user enters this arc at time $t$. Any nonlinear travel time function can be incorporated in this model as linear constraints. Hence, a linear travel time function is used as follows,

$$
\phi(\text { flow })=a *\left[1+b *\left(\frac{\text { flow }}{c}\right)^{p}\right]
$$

where $a$ is the free flow travel time, $b$ is a coefficient, $c$ is the capacity of the link, $p$ is the power, and they are all equated as a function of flow on each respective arc. Regardless of departure time, there should be a limit/bound on users minimum and maximum travel time on an arc. It is a fair assumption that free flow travel time $a$ is regarded as users' 
minimum possible travel time on an arc for this model. On the other hand, maximum flow is used to decide maximum travel time of an arc through the following equation:

$$
\phi_{t}^{p, q}\left(\text { flow }_{\max }\right)=a *\left[1+b *\left(\frac{\text { flow }_{\max }}{c}\right)^{p}\right]
$$

The maximum flow on an arc is closely related to the capacity. Hence, it it important to first understand how road capacities are assessed in order to derive a sensible estimate of maximum flow. Road capacity is essentially the maximum number of vehicles can expect to traverse a point of a roadway during a given period under prevailing roadway traffic conditions [72]. Road capacities are stochastic in nature and there exist different meanings to capacity value notions [73]: 1. Design Capacity, 2. Strategic Capacity, and 3. Operational Capacity. Although several capacity-estimation methods exist (some more promising than others), a reliable and meaningful estimate of road capacity does not appear to be available [73]. However, some conditions and rules stay constant when traffic engineers conduct road capacity estimations [73]. Firstly, uninterrupted roadway conditions are used when estimating capacity. Secondly, traffic data with which capacity of a road is estimated should be collected at one or more cross sections of the road. Congestion typically begins upstream of the bottleneck. Hence, observation points will need to take place at upstream, bottleneck, and downstream areas of a road segment. Figure 3.5 illustrates the various measuring points on an arc. In this research, capacity is assumed to be the maximum number of vehicles that is on a road segment at any given time under the two constant conditions stated earlier. As such, longer road segments will be able to contain more vehicles on it, in turn it also takes more vehicles to be on it to achieve congestion state. In other words, longer road segments respectively will have higher capacity.

Since roadway capacities are estimated under uninterrupted conditions, it is assumed that the maximum flow during an emergency evacuation will be comparatively near to the capacity limit and in many situations, even surpassing the capacity limit significantly. Also, since longer road segments will have higher capacities, it is assumed that maximum 


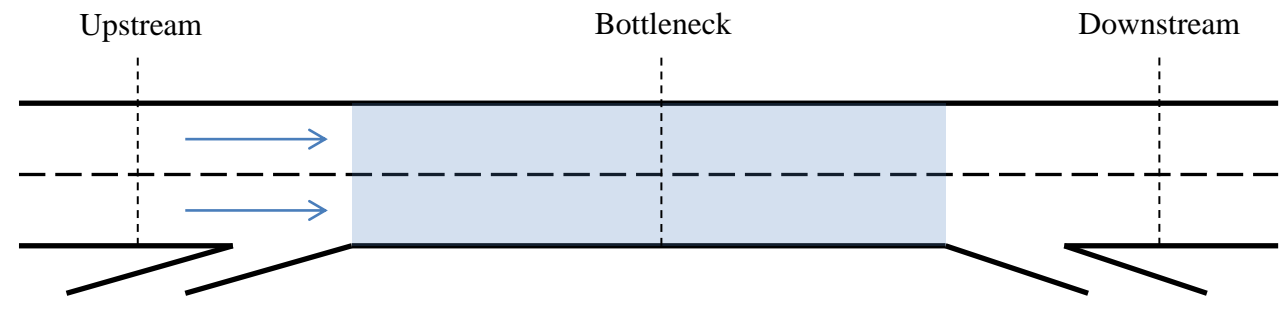

Figure 3.5: Capacity measuring point.

flow during an emergency evacuation will consist of a higher percentage of the capacity limit. As such, the percentage of capacity for maximum flow is taken to be linearly correlated to the length of the road segment. Figure 3.6 shows that maximum flow is $90 \%$ of capacity for the shortest road segment in the network and $200 \%$ for the longest.

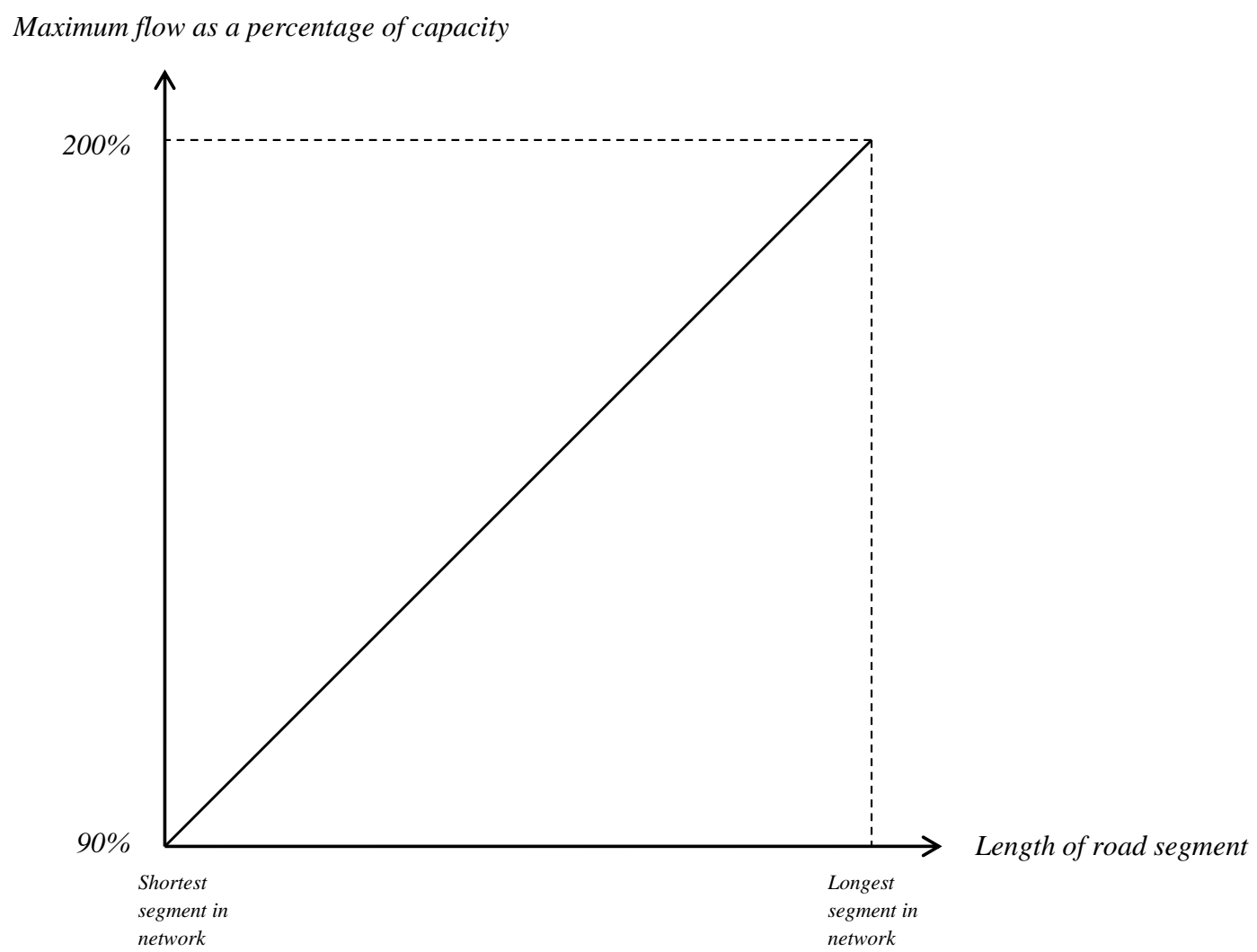

Figure 3.6: Relationship between maximum flow and length of road segment. 


\subsection{Travel Time}

Usually the set of possible discrete travel times of segment $(p, q), \Delta_{p, q}$, contains more than one element. Exactly one time expanded arc will be realized among the set of arcs emanating from any time expanded node, since it is assumed that all users entering an arc in the same time interval will experience the same travel time on this arc. Hence, the set of binary variables $z_{p, q ; t, t+\tau}$ are introduced to help model the realization of time expanded arc $(p, q ; t, t+\tau)$ where

$z_{p, q ; t, t+\tau}= \begin{cases}1, & \text { Travel time } \tau \text { on }(p, q) \text { when enter arc from node } p \text { at time } t \\ 0, & \text { Otherwise. }\end{cases}$

The same applies for reversed flow:

$z_{p, q ; t, t+\tau}^{\prime}= \begin{cases}1, & \text { Travel time } \tau \text { on }(p, q) \text { when enter arc from node } q \text { at time } t \\ 0, & \text { Otherwise. }\end{cases}$

Let $y_{p, q ; t, t+\tau}$ be the flow variable and $y_{p, q ; t, t+\tau}^{\prime}$ be the contra-flow variable on time expanded arc $(p, q ; t, t+\tau)$. In order to enforce the unique realization of travel time, the following constraints for regular and reversed flow need to be included in the formulation,

$$
\begin{aligned}
& y_{p, q ; t, t+\tau} \leq M_{p, q} z_{p, q ; t, t+\tau}, \quad \forall \tau \in \Delta_{p, q}, \\
& y_{p, q ; t, t+\tau}^{\prime} \leq M_{p, q} z_{p, q ; t, t+\tau}^{\prime}, \quad \forall \tau \in \Delta_{p, q},
\end{aligned}
$$

where $M_{p, q}$ is the upper bound on the number of vehicles that can enter arc $(p, q)$ during a time period.

Here, it is assumed that the travel time on any static arc $(p, q)$ is time dependent. $\phi_{t}^{p, q}$ is denoted to be the travel time on $\operatorname{arc}(p, q)$ when a user enters this arc at time $t$. In reality, $\phi_{t}^{p, q}$ is determined by all vehicles which are currently on or are entering the road. Then the assumption is made that any users traveling speed is only affected by the vehicles ahead of and around the user but not those behind the user. Hence, $\phi_{t}^{p, q}$ 
should be a multivariate function of all positive flows on time expanded arcs at time $t$, which means the vehicles that are still on the road at this time point. The function can be expressed as follows,

$$
\phi_{t}^{p, q}=\phi_{t}^{p, q}\left(y_{p, q ; \alpha, \beta},\{(\alpha, \beta): \alpha \leq t, \beta \geq t\}\right) .
$$

Intuitively, closer vehicles have bigger effects on traveling speed, as shown Figure 3.7, where the darker is the dot, the larger is the effect.

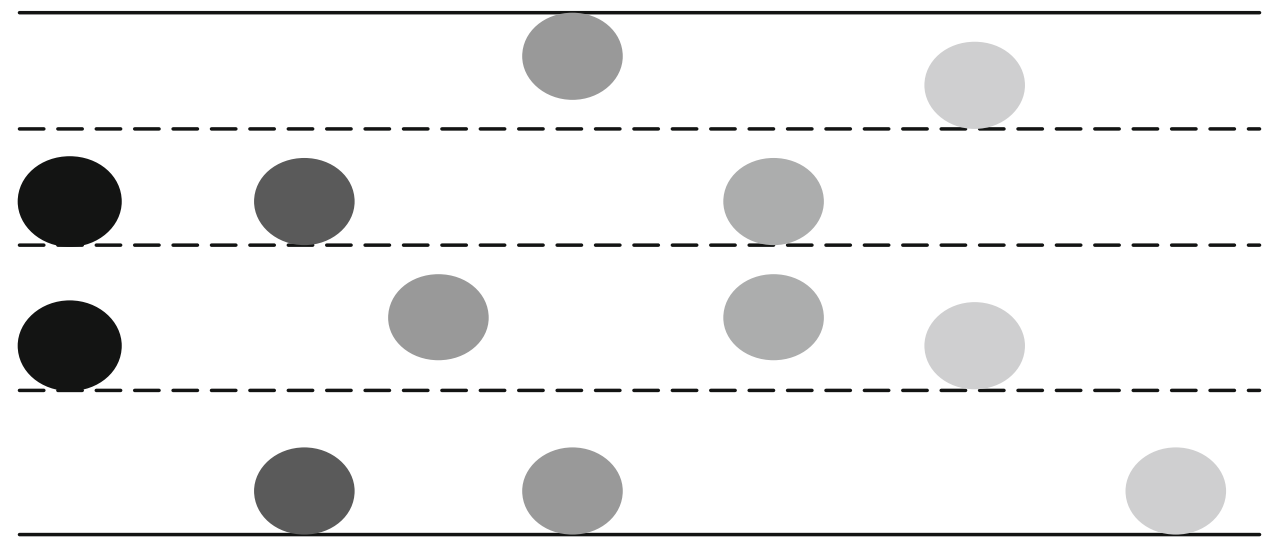

Figure 3.7: Effectiveness of vehicle positions on travel time [5].

For convenience, a single variable function is used to calculate the travel time. The single variable is weighted flow, $x_{t}^{p, q}$, and the travel time for the vehicles which enter $(p, q)$ at time $t$ is as follows,

$$
\phi_{t}^{p, q}=\phi_{t}^{p, q}\left(\sum_{\alpha \leq t} \sum_{\beta \geq t} y_{p, q ; \alpha, \beta}\right)=\phi_{t}^{p, q}\left(x_{t}^{p, q}\right) .
$$

The travel time on a static arc determines the realization of the time expanded arcs. On the other hand, the realization of the time expanded arcs decides the weighted flows at discrete time points. In order to capture this feature, the following constraints for regular flow are included in the model, 


$$
\sum_{\tau \in \Delta_{p, q}}(\tau-\delta) z_{p, q ; t, t+\tau}<\phi_{t}^{p, q}\left(x_{t}^{p, q}\right) \leq \sum_{\tau \in \Delta_{p, q}} \tau z_{p, q ; t, t+\tau}, \quad \forall t \in \Gamma ;(p, q) \in A
$$

which means that the travel time on time expanded $\operatorname{arc}(p, q ; t, t+\tau)$, when entering at time $t$, is greater than $\tau-\delta$ and less than or equal to $\tau$. The travel time function is assumed to be a continuous and non-decreasing function as shown in Figure 3.8, and then its inverse function is also continuous and non-decreasing. Hence, since $z_{p, q ; t, t+\tau}$ is a binary variable, the above nonlinear constraints can be transformed to the equivalent linear constraints as follows,

$$
\sum_{\tau \in \Delta_{p, q}} \phi_{p, q}^{-1}(\tau-\delta) z_{p, q ; t, t+\tau}<x_{t}^{p, q} \leq \sum_{\tau \in \Delta_{p, q}} \phi_{p, q}^{-1}(\tau) z_{p, q ; t, t+\tau}, \quad \forall t \in \Gamma ;(p, q) \in A
$$

The same concept applies for reversed flows, where the bounds of its inverse travel time function is shown as

$$
\sum_{\tau \in \Delta_{p, q}} \phi_{p, q}^{-1}(\tau-\delta) z_{p, q ; t, t+\tau}^{\prime}<x_{t}^{\prime p, q} \leq \sum_{\tau \in \Delta_{p, q}} \phi_{p, q}^{-1}(\tau) z_{p, q ; t, t+\tau}^{\prime}, \quad \forall t \in \Gamma ;(p, q) \in A
$$

As an example, the inverse travel time (inverse of Equation 3.1) upper bound is a function of travel time $\tau$,

$$
\sum_{\tau \in \Delta_{p, q}} \phi_{p, q}^{-1}(\tau)=\sum_{\tau \in \Delta_{p, q}} \sqrt[p]{\frac{\tau}{a * b}-\frac{1}{b}} * c
$$

This implies that if values of $z_{p, q ; t, t+\tau} \mathrm{S}$ and $z_{p, q ; t, t+\tau}^{\prime} \mathrm{S}$ are given, the upper and lower bounds on the weighted flow, $x_{t}^{p, q}$, and reversed weighted flow, $x_{t}^{\prime p, q}$, are also given. 


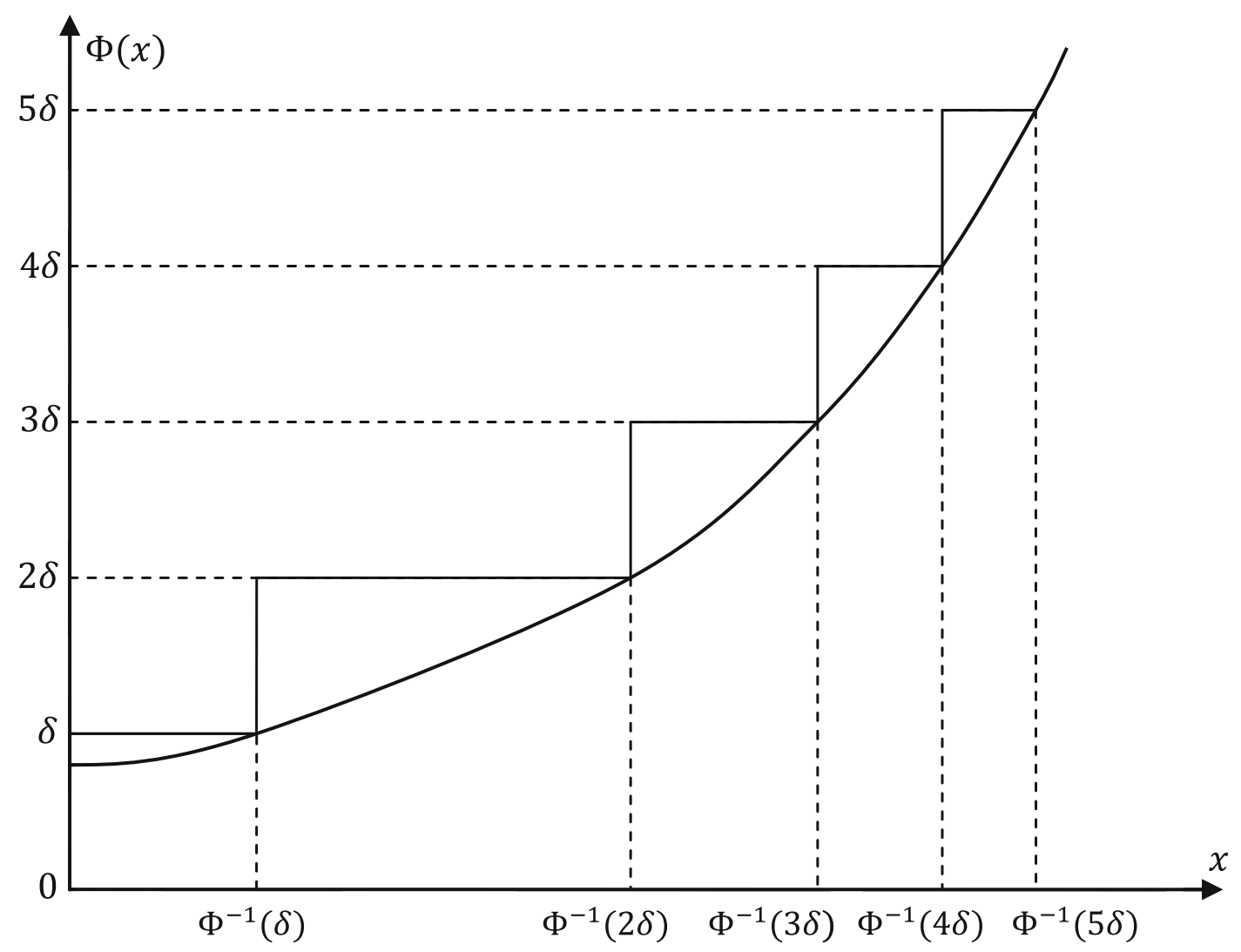

Figure 3.8: Travel time function [5].

\subsection{Demand Generation}

Demand from every node $i, h_{i}$, is a parameter that will be input in the model. Demand will then be converted as starting/beginning flow at every node. The variable $w_{i, t}$ is denoted as starting flow from node $i$ departing at time $t$. The total demand at every node is equal to the total starting flow departing at every time period shown as follows,

$$
\sum_{t} w_{i, t}=h_{i}, \quad \forall t \in A
$$

In an evacuation scenario, there are safety areas designated for evacuees to head towards for refuge. These designated safe areas are denoted as safe nodes $s$. The variable $v_{p, s ; t}$ denotes sink flow that departs from node $p$ at time $t$ heading for safe node $s$. Safe nodes are assumed to be sink nodes where they absorb all sink flows that lead to them. The 
following constraint captures this feature,

$$
\sum_{(p, s) \in A} \sum_{t} v_{p, s ; t}=h_{s}
$$

As demand for every node $h_{i}$ is set as a parameter, naturally, total demand $\sum_{i} h_{i}$ will be equal to the total sink demand, denoted as $h_{s}$.

\subsection{Flow Balance}

Flow balance condition for any kind of network is crucial in ensuring correctness and feasibility when computing results. Great care must be taken especially when formulating flow balance of a time expanded network, the possibility of lane reversals also adds on to its complication. The general rule of thumb when formulating is that for every node, at every time point, all flows coming in must equal all flows going out,

$$
\begin{aligned}
& \sum_{q}\left(\sum_{\tau} y_{p, q ; t, t+\tau}-\sum_{\alpha} y_{p, q ; \alpha, \beta}^{\prime}\right)+\sum_{o}\left(\sum_{\tau} y_{o, p ; t, t+\tau}^{\prime}-\sum_{\alpha} y_{o, p ; \alpha, \beta}\right)=0 \\
& \beta=t ; \forall t, \beta \in \Gamma ; \forall i, p \in A
\end{aligned}
$$

Possible outgoing flows from node $p$ at time $t$ are represented by variable $y_{p, q ; t, t+\tau}$, which shows flow heading for nodes ahead $q$. Also, reversed flow-out heading for previous nodes $o$, represented by variable $y_{o, p ; t, t+\tau}^{\prime}$, needs to be included in as part of flow-out values. Similarly for incoming flows, variable $y_{o, q ; \alpha, \beta}$ is used to represent flow-in from previous nodes and variable $y_{p, q ; \alpha, \beta}^{\prime}$ used to represent reversed flow-in from nodes ahead. In this case, all departure time $\alpha$ needs to be less than $t$ and arrival time $\beta$ equal to $t$. Figure 3.9 illustrates a flow balance condition example for node $p$ at time point $2 \delta$, where the solid lines represent regular flow and dotted lines represent reversed flow.

Demand at each node is converted to start flow at different time points $w_{i, t}$ according to Equation 3.9. As such, $w_{i, t}$ is also taken as flow-in in the flow balance constraint. It 


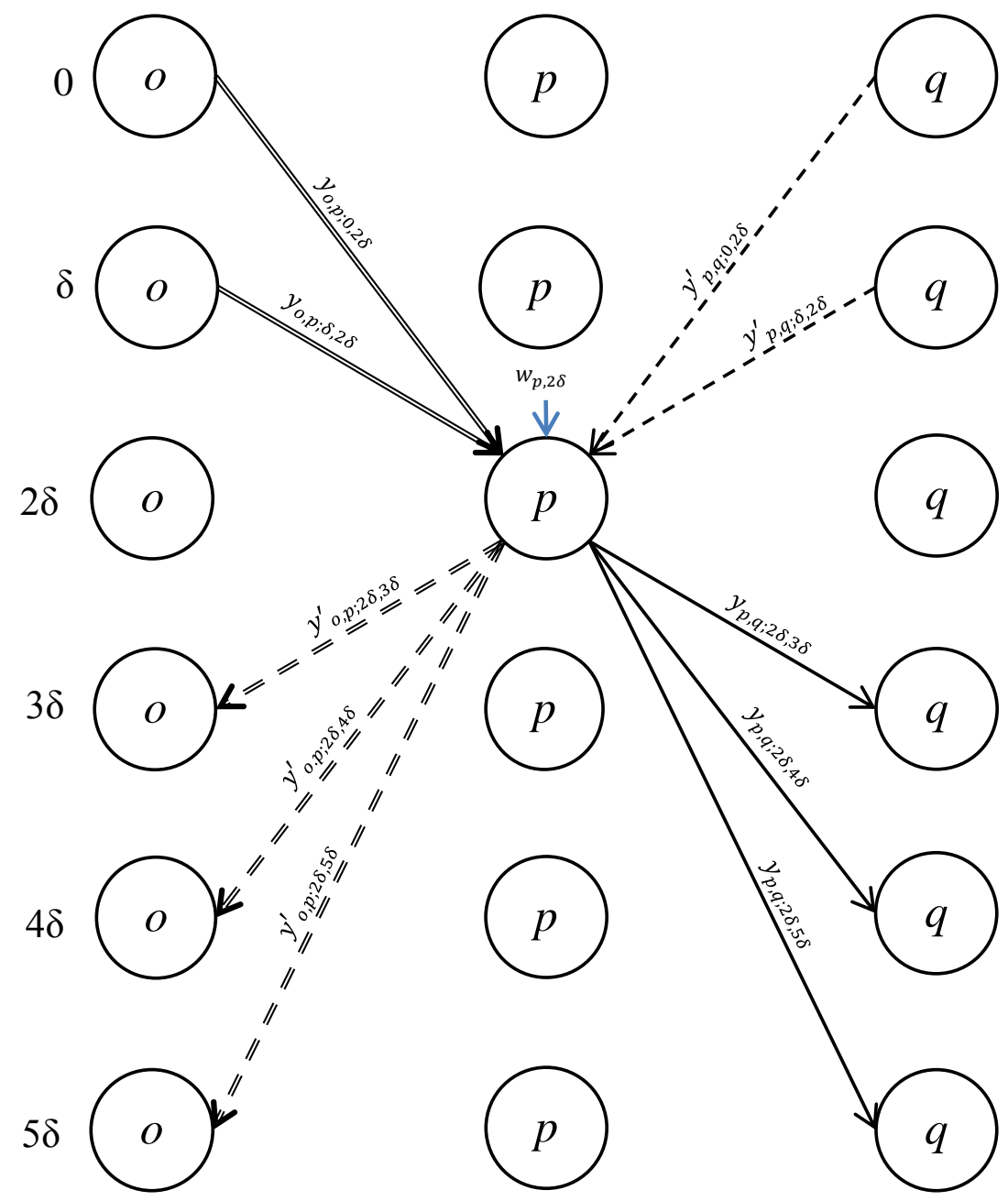

Figure 3.9: Flow balance condition.

is assumed that reversed flow is prohibited when it comes to flow heading for safe nodes $s$. Hence for ending flow equation, only end-flow $v_{p, s ; t}$, reverse flow-out to previous nodes $y_{o, p ; t, t+\tau}^{\prime}$, and flow in from previous nodes $y_{o, p ; \alpha, \beta}$ are considered,

$$
\begin{aligned}
& \sum_{(p, s) \in A} \sum_{t} v_{p, s ; t}+\sum_{o} \sum_{\tau} y_{o, p ; t, t+\tau}^{\prime}-\sum_{o} \sum_{\alpha} y_{o, p ; \alpha, \beta}=0 \\
& \beta=t ; \forall t, \beta \in \Gamma ; \forall p \in A .
\end{aligned}
$$




\subsection{Collision Prevention}

With the possibility of lane reversals/contraflow being realized, the threat of potential head on collision with oncoming traffic naturally emerges. To prevent the occurrence of such an event, total clearance of regular-directional flow on an arc $(p, q)$ needs to be ensured before the arc is reversed. To help prevent collisions of vehicles, the following constraint is needed,

$$
\sum_{\tau} z_{p, q ; t, t+\tau}+\sum_{\beta} z_{p, q ; \alpha, \beta}^{\prime} \leq 1, \quad \forall \alpha<t+\xi ; \xi \in \tau ; \forall \beta>t ; \forall t \in \Gamma_{p, q}
$$

where $z_{p, q ; t, t+\tau}$ represents realization of regular flow for time expanded arc $(p, q ; t, t+\tau)$ and $z_{p, q ; \alpha, \beta}^{\prime}$ represents realization of reversed flow for time expanded arc $(p, q ; \alpha, \beta)$. As such, equation 3.13 shows that the sum of all regular flows (regardless of travel time $\tau$ ) on $\operatorname{arc}(p, q)$ departing at time $t$, and the sum of all reversed flow on the same arc which has departure time $\alpha$ less than earliest arrival time of regular flow $t+\xi$ ( $\xi$ is quickest travel time in this case) and arrival time $\beta$ greater than departure time of regular flow $t$, are less than or equal to 1. Figure 3.10 illustrates Equation 3.13 graphically.

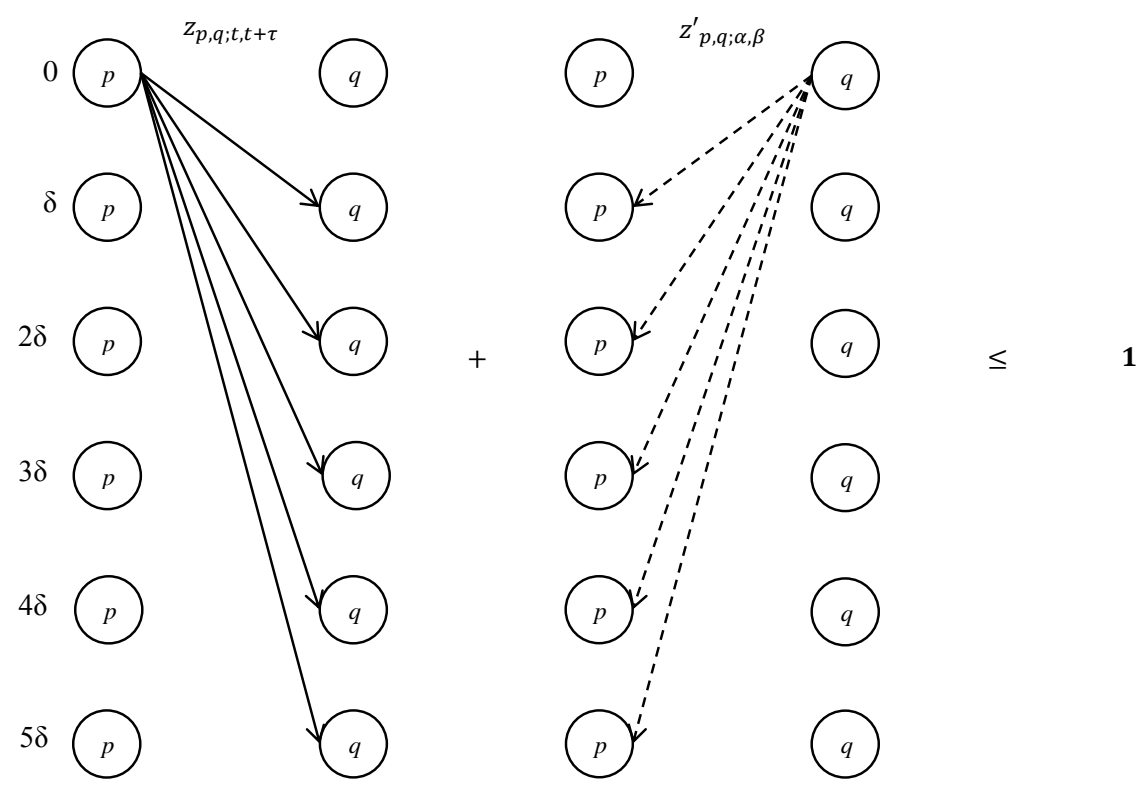

Figure 3.10: Collision prevention constraint graphically. 
Since $\alpha<t+\xi$, in situations where earliest arrival time for regular flow $t+\xi$ does not equal to next immediate period of its departure time $t+\delta$, there will be in turn more than one reversed flow departure time $\alpha$ that will potentially violate the collision prevention condition. To tackle this problem, travel time for regular flow is placed within the set $\Delta_{p, q}(\xi)$ where

$$
\Delta_{p, q}(\xi)=\Delta_{p, q} \backslash\left\{\delta_{m}, \delta_{m}+\delta, \ldots, \xi-\delta\right\}=\left\{\xi, \xi+\delta, \ldots, \delta^{\max }\right\}
$$

$\delta_{m}$ in this case is denoted as the minimum travel time. Combined with Equation 3.13, the collision prevention constraint will take the following form,

$$
\begin{aligned}
& \sum_{\tau \in \Delta_{p, q}(\xi)} z_{p, q ; t, t+\tau}+\sum_{\beta \in T C(t)} z_{p, q ; \alpha, \beta}^{\prime} \leq 1, \quad \forall \alpha<t+\xi ; \forall t \in \Gamma_{p, q} ; \forall \xi \in \Delta_{p, q} \\
& \Delta_{p, q}(\xi)=\Delta_{p, q} \backslash\left\{\delta_{m}, \delta_{m}+\delta, \ldots, \xi-\delta\right\}=\left\{\xi, \xi+\delta, \ldots, \delta^{\text {max }}\right\} \\
& T C(t)=\{\beta \mid \beta>t\}
\end{aligned}
$$

To further understand collision prevention constraint, Figure 3.11 shows an example when quickest regular flow travel time is $2 \delta$, there exist two sets of collision prevention constraints, one with reversed flow departure time 0 , and the other with reversed flow departure time $\delta$. When earliest regular flow arrival time shifts to $3 \delta$, an additional set of constraint emerges, namely reversed flow with departure time $2 \delta$, as can be seen in Figure 3.12 .

The number of collision prevention constraints that emerges will increase according to the number of time periods assigned to the model. The earliest arrival time and quickest travel time as shown, are related to each other. Both are also dependent upon the minimum travel time on each arc as explained in Section 3.2. On top of restricting directional flow, the collision prevention constraint also ensures only one time expanded arc is realized for every static arc $(p, q)$, for both regular and reversed flow. This is also referred to as unique realization condition. 

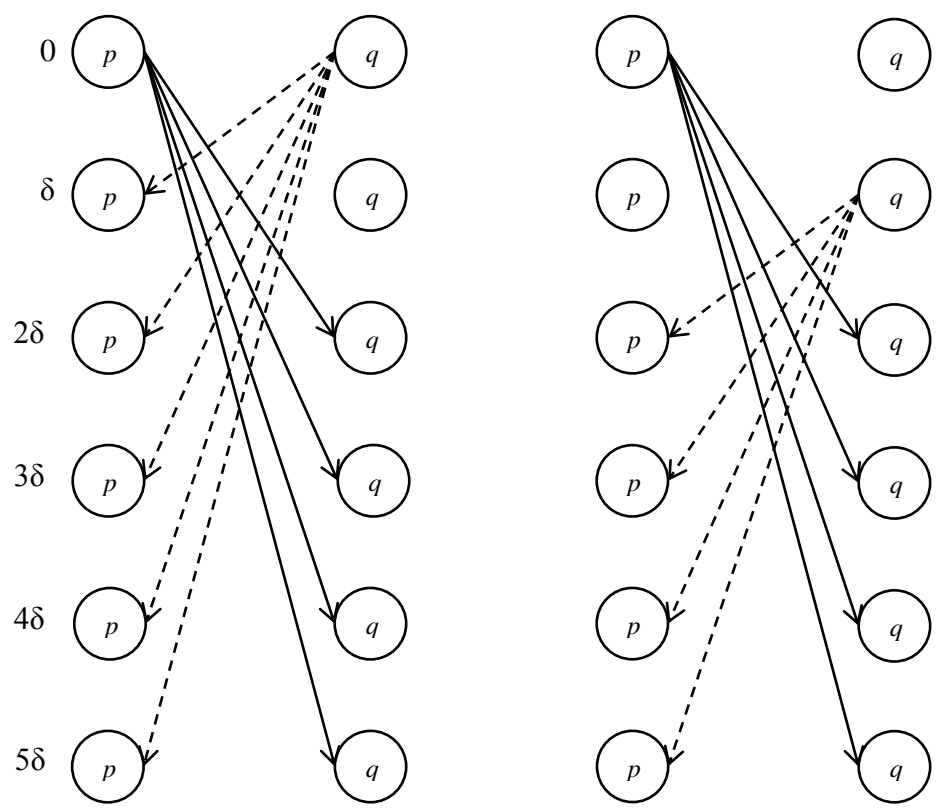

Figure 3.11: Collision prevention with quickest regular flow travel time equal $2 \delta$.
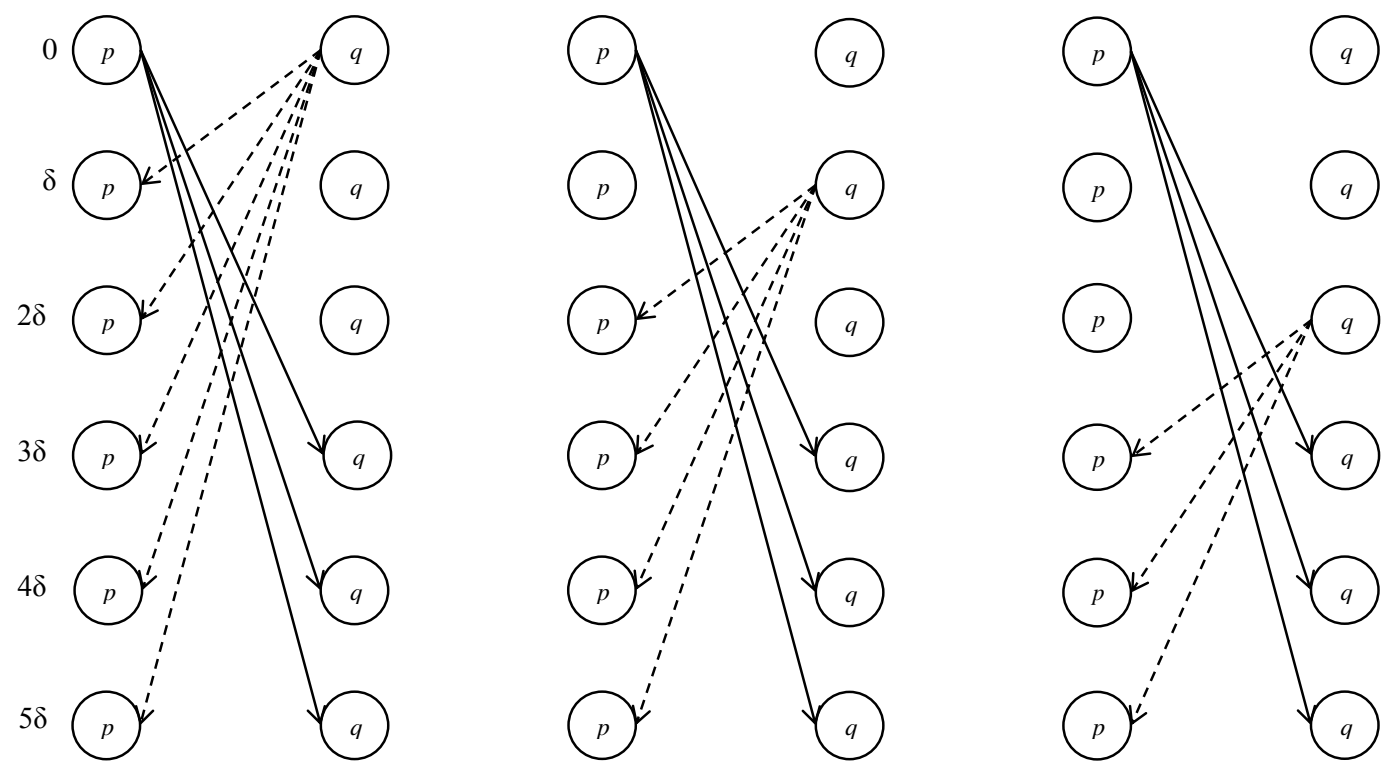

Figure 3.12: Collision prevention with quickest regular flow travel time equal $3 \delta$.

\subsection{Mathematical Formulation}

Before getting into the formulation, users departure time need to be discussed. As simultaneous route and departure (SRD) assumption is incorporated in this model, the actual departure times can be different from desired ones. Hence, appropriate penalties 
have to be added if there exist any differences between desired departure times and actual ones. In addition to the aforementioned notations and variables, a detailed list of all indices, variables, and parameters are given in Tables 3.1, 3.2, and 3.3. Based on all previously defined constraints and sets together with their variables, the system optimum problem for emergency evacuation planning is formulated as follows,

$$
\begin{aligned}
\operatorname{Min} & \sum_{(p, q) \in A} \sum_{t \in \Gamma} \phi_{p, q}\left(x_{t}^{p, q}\right) \sum_{\tau \in \Delta_{p, q}} y_{p, q ; t, t+\tau}+\sum_{(p, q) \in A} \sum_{t \in \Gamma} \phi_{p, q}\left(x_{t}^{\prime p, q}\right) \sum_{\tau \in \Delta_{p, q}} y_{p, q ; t, t+\tau}^{\prime} \\
& +\sum_{i} \sum_{t} t \cdot w_{i, t}
\end{aligned}
$$

s.t. $\sum_{t} w_{i, t}=h_{i}, \quad \forall i \in A$

$\sum_{(p, s) \in A} \sum_{t} v_{p, s ; t}=h_{s}$

$$
\sum_{q}\left(\sum_{\tau} y_{p, q ; t, t+\tau}-\sum_{\alpha} y_{p, q ; \alpha, \beta}^{\prime}\right)+\sum_{o}\left(\sum_{\tau} y_{o, p ; t, t+\tau}^{\prime}-\sum_{\alpha} y_{o, p ; \alpha, \beta}\right)
$$$$
-w_{i, t}=0, \quad \beta=t ; \forall t, \beta \in \Gamma ; \forall i, p \in A
$$

$\sum_{q}\left(\sum_{\tau} y_{p, q ; t, t+\tau}-\sum_{\alpha} y_{p, q ; \alpha, \beta}^{\prime}\right)+\sum_{o}\left(\sum_{\tau} y_{o, p ; t, t+\tau}^{\prime}-\sum_{\alpha} y_{o, p ; \alpha, \beta}\right)=0$,

$\beta=t ; \forall t, \beta \in \Gamma ; \forall i, p \in A$

$\sum_{(p, s) \in A} \sum_{t} v_{p, s ; t}+\sum_{o} \sum_{\tau} y_{o, p ; t, t+\tau}^{\prime}-\sum_{o} \sum_{\alpha} y_{o, p ; \alpha, \beta}=0$,

$\beta=t ; \forall t, \beta \in \Gamma ; \forall p \in A$

$x_{t}^{p, q}=\sum_{\alpha} \sum_{\beta} y_{p, q ; \alpha, \beta}, \quad \forall t \in \Gamma ;(p, q) \in A ; \alpha \leq t, \beta \geq t$

$x_{t}^{\prime p, q}=\sum_{\alpha} \sum_{\beta} y_{p, q ; \alpha, \beta}^{\prime}, \quad \forall t \in \Gamma ;(p, q) \in A ; \alpha \leq t, \beta \geq t$

$x_{t}^{p, q} \geq \sum_{\tau \in \Delta_{p, q}} \phi_{p, q}^{-1}(\tau-\delta) z_{p, q ; t, t+\tau}, \quad \forall t \in \Gamma_{p, q} ;(p, q) \in A$

$x_{t}^{\prime p, q} \geq \sum_{\tau \in \Delta_{p, q}} \phi_{p, q}^{-1}(\tau-\delta) z_{p, q ; t, t+\tau}^{\prime}, \quad \forall t \in \Gamma_{p, q} ;(p, q) \in A$

$x_{t}^{p, q} \leq \sum_{\tau \in \Delta_{p, q}} \phi_{p, q}^{-1}(\tau) z_{p, q ; t, t+\tau}, \quad \forall t \in \Gamma_{p, q} ;(p, q) \in A$ 


$$
\begin{aligned}
& x_{t}^{\prime p, q} \leq \sum_{\tau \in \Delta_{p, q}} \phi_{p, q}^{-1}(\tau) z_{p, q ; t, t+\tau}^{\prime}, \quad \forall t \in \Gamma_{p, q} ;(p, q) \in A \\
& y_{p, q ; t, t+\tau} \leq M_{p, q} z_{p, q ; t, t+\tau}, \quad \forall t \in \Gamma_{p, q} ; \tau \in \Delta_{p, q} ;(p, q) \in A \\
& y_{p, q ; t, t+\tau}^{\prime} \leq M_{p, q} z_{p, q ; t, t+\tau}^{\prime}, \quad \forall t \in \Gamma_{p, q} ; \tau \in \Delta_{p, q} ;(p, q) \in A \\
& \quad \sum_{\tau \in \Delta_{p, q}(\xi)} z_{p, q ; t, t+\tau}+\sum_{\beta \in T C(t)} z_{p, q ; \alpha, \beta}^{\prime} \leq 1, \quad \forall \alpha<t+\xi ; \forall t \in \Gamma_{p, q} ; \forall \xi \in \Delta_{p, q} \\
& \quad \Delta_{p, q}(\xi)=\Delta_{p, q} \backslash\left\{\delta_{m}, \delta_{m}+\delta, \ldots, \xi-\delta\right\}=\left\{\xi, \xi+\delta, \ldots, \delta^{m a x}\right\} \\
& \quad T C(t)=\{\beta \mid \beta>t\} \\
& v, w, x, y, x^{\prime}, y^{\prime} \geq 0 \\
& z_{p, q ; t, t+\tau} \in\{0,1\}, \quad \forall t \in \Gamma_{p, q} ; \tau \in \Delta_{p, q} ;(p, q) \in A \\
& z_{p, q ; t, t+\tau}^{\prime} \in\{0,1\}, \quad \forall t \in \Gamma_{p, q} ; \tau \in \Delta_{p, q} ;(p, q) \in A
\end{aligned}
$$

Table 3.1: Indices and definitions.

\begin{tabular}{|l|l|}
\hline \hline \multicolumn{1}{|c|}{ Notation } & \multicolumn{1}{c|}{ Definition } \\
\hline$\Gamma$ & Set of discrete time points within planning time horizon \\
\hline$t$ & Departure time of users where $t \in \Gamma$ \\
\hline$A$ & Set of static arcs \\
\hline$\Delta_{p, q}$ & All possible discrete travel times for arc $(p, q) \in A$ \\
\hline$\tau$ & Traveling time of users where $\tau$ belongs to $\Delta_{p, q}$ \\
\hline$(p, q ; t, t+\tau)$ & Realization of static arc $(p, q)$ departing at time $t$ with travel time $\tau$ \\
\hline$\alpha$ & Departure time of incoming flow \\
\hline$\beta$ & Arrival time of incoming flow \\
\hline$\delta^{\text {min }}$ & Minimum travel time \\
\hline$\delta^{\text {max }}$ & Maximum travel time \\
\hline
\end{tabular}

Demand generation and flow absorbtion is shown in constraints $3.17 \mathrm{a}$ and $3.17 \mathrm{~b}$. The flow balance of time expanded network is represented by constraints $3.17 \mathrm{c}, 3.17 \mathrm{~d}$ and 3.17e. Constraints $3.17 \mathrm{f}$ and $3.17 \mathrm{~g}$ calculates the weighted flows. Constraints $3.17 \mathrm{~h}$ and $3.17 \mathrm{i}$ model the lower bound of regular and reversed flow travel time. Constraints $3.17 \mathrm{j}$ and $3.17 \mathrm{k}$ model the upper bound of regular and reversed flow travel time. Constraint 3.17l and $3.17 \mathrm{~m}$ ensures the unique travel time for each static arc at any time point. Collision prevention between regular flow and reversed flow is realized by constraint $3.17 \mathrm{n}$. For convenience, $x$ denotes the decision vector composed of $x_{t}^{p, q}$. So does decision vectors $x^{\prime}$, $w, v, y$, and $y^{\prime}$. 
Table 3.2: Decision variables and definitions.

\begin{tabular}{|l|l|}
\hline \hline \multicolumn{1}{|c|}{ Notation } & \multicolumn{1}{c|}{ Definition } \\
\hline$y_{p, q ; t, t+\tau}$ & Flow on time expanded arc $(p, q ; t, t+\tau)$ \\
\hline$y_{p, q ; t, t+\tau}^{\prime}$ & Reverse flow on time expanded arc $(p, q ; t, t+\tau)$ \\
\hline$w_{i, t}$ & Start flow at time $t$ from node $i$ \\
\hline$v_{t}$ & Ending flow at time $t$ that leads to sink node \\
\hline$z_{p, q ; t+\tau}$ & $\begin{array}{l}\text { Set of binary variables that model realization of flow on time expanded arc } \\
(p, q ; t, t+\tau)\end{array}$ \\
\hline$z_{p, q ; t+\tau}^{\prime}$ & $\begin{array}{l}\text { Set of binary variables that model realization of reverse flow on time ex- } \\
\text { panded arc }(p, q ; t, t+\tau)\end{array}$ \\
\hline$x_{t}^{p, q}$ & Weighted flow at discrete time points \\
\hline$x_{t}^{\prime p, q}$ & Reverse weighted flow at discrete time points \\
\hline
\end{tabular}

Table 3.3: Parameters and definitions.

\begin{tabular}{|l|l|}
\hline \hline Notation & \multicolumn{1}{c|}{ Definition } \\
\hline$M_{p, q}$ & Upper bound on number of flow that can enter arc $(p, q)$ during a time period \\
\hline$\phi_{p, q}$ & $\begin{array}{l}\text { Multivariate, continuous, and non-decreasing travel time function on arc } \\
(p, q) \text { at time } t\end{array}$ \\
\hline$\phi_{p, q}^{-1}$ & Inverse of travel time function \\
\hline
\end{tabular}

The system optimum DTA (DTA-SO) is a mixed integer nonlinear program, where nonlinearity only exists in the objective function. The objective function of the model is split into two parts: the linear and nonlinear part. The penalties for late departures form the linear part since penalty for any specific discrepancy between actual and desired times are predetermined. The remaining part is nonlinear since travel time on arc $(p, q)$, when departing at time $t$, is generally a nonlinear function of weighted flow, $x_{t}^{p, q}$ and $x_{t}^{\prime p, q}$. Based on constraint $3.17 \mathrm{j}$ and $3.17 \mathrm{k}$, the following is obtained,

$$
\begin{aligned}
& \phi_{p, q}\left(x_{t}^{p, q}\right) \leq \sum_{\tau \in \Delta_{p, q}} \tau \cdot z_{p, q ; t, t+\tau}, \quad \forall(p, q) \in A, t \in \Gamma \quad \text { and } \\
& \phi_{p, q}\left(x_{t}^{\prime p, q}\right) \leq \sum_{\tau \in \Delta_{p, q}} \tau \cdot z_{p, q ; t, t+\tau}^{\prime}, \quad \forall(p, q) \in A, t \in \Gamma .
\end{aligned}
$$

Together with constraints $3.17 \mathrm{l}$ and $3.17 \mathrm{~m}$, the following inequalities are obtained, 


$$
\begin{aligned}
& \phi_{p, q}\left(x_{t}^{p, q}\right) \sum_{\tau \in \Delta_{p, q}} y_{p, q ; t, t+\tau} \leq \sum_{\tau \in \Delta_{p, q}} \tau \cdot y_{p, q ; t, t+\tau}, \quad \forall(p, q) \in A, t \in \Gamma \quad \text { and } \\
& \phi_{p, q}\left(x_{t}^{\prime p, q}\right) \sum_{\tau \in \Delta_{p, q}} y_{p, q ; t, t+\tau}^{\prime} \leq \sum_{\tau \in \Delta_{p, q}} \tau \cdot y_{p, q ; t, t+\tau}^{\prime}, \quad \forall(p, q) \in A, t \in \Gamma .
\end{aligned}
$$

This gives the upper bound on the nonlinear part of the original objective function of the DTA-SO. Hence, we can define the upper bound of the objective function as follows,

$$
\sum_{(p, q) \in A} \sum_{t \in \Gamma} \sum_{\tau \in \Delta_{p, q}}\left[\tau \cdot y_{p, q ; t, t+\tau}+\tau \cdot y_{p, q ; t, t+\tau}^{\prime}\right]+\sum_{i} \sum_{t} t \cdot w_{i, t}
$$

The above function together with all the constraints in the original DTA-SO problem, form the upper bound problem, DTA-UB. For convenience, hereafter, the vector format $c_{1}^{T} w+c_{2}^{T} y$ is used as the upper bound objective function, since all costs are prefixed. $U B$ and $S O$ are the optimal objective values of the DTA-UB and DTA-SO, respectively. Nahapetyan and Lawphongpanich [71] proposed a theorem regarding relationship between $U B$ and $S O$.

Theorem 1 Given any $\varepsilon>0$, there exist a $\delta>0$ such that $U B-S O<\varepsilon$.

The proof can be found in their paper [71]. Even though their objective functions do not include any penalties, Theorem 1 still applies in this situation because the penalty parts in both DTA-UB and DTA-SO are the same. Hence, instead of having to solve the original nonlinear problem, the linearized upper bound problem can instead be solved which gives a very good approximation. The final model will then be a mixed integer linear programming problem.

\subsection{Model Assumptions and Limitations}

In the process of modeling, like most formulation of mathematical models, some assumptions need to be made for it fit the situation to achieve the purposed that is set. With that said, no assumption can be made wildly without a firm basis that causes the model to lose its validity. However, some extent of compromise is unavoidable when making 
assumptions, although these must logically conform to possible real-life cases.

This model implements link FIFO (first-in, first-out) rule. This means that all vehicles entering the same arc/link at the same time point, will experience the same travel time. This implies that there is no overtaking between vehicles, even when vehicles exit link by different turning movements. This is especially likely to occur during massive congestions such as during an emergency evacuation. O-D flows and roadway characteristics are assumed to be fixed and known. Some examples such as information regarding free flow time $a$, road capacity $c$, and roadway availability are obtained and fixed as inputs and do not change throughout the evacuation period. Also, in computing optimal route choice and departure time, road users/evacuees at every node are assumed to be fixed and unchanging. As mentioned in Section 3.2, maximum flow for every link is dependent upon capacity, which in turn is assumed to be linearly dependent upon the length of its respective link. Reasoning behind such an assumption is stated within the same section above. Modeling time horizon is assigned to be sufficient for all evacuees to discover shortest travel time to evacuate. Otherwise, there will be a possibility of an infeasible output when computing. In other words, this model strives to achieve a $100 \%$ of evacuees evacuated to safe zones and does not take into account partial evacuation or available shelter areas within danger zones for evacuees that is unable to make it to safe zones in time. Finally, all safe nodes are assumed to be limitless in their capacity. In other words, they are able to absorb however much flow that are assigned to them. In reality, this might be possible if total evacuees are relatively small in number, but will be infeasible if they are extravagantly large in amount. This research additionally solves the mathematical model using a deterministic approach that utilizes CPLEX solver to obtain absolute optimal solution. Additionally, other available techniques such as simulation or metaheuristics, were not tested. Although some of these techniques might not provide absolute optimal solutions, they might generate relatively close to optimal solutions with significantly less computational time.

There definitely exist room for future research to tighten these assumption or incorporate certain aspects in the model that tackles these areas. Nevertheless, there is an expression about modeling that is good to keep in mind: "All models are wrong and imperfect, but some are useful" [74]. It is the authors' challenge to formulate a good model that is useful and make good predictions to improve emergency evacuation planning. 


\section{Chapter 4}

\section{Model Analysis}

The purpose of this chapter is to analyze the DTA model that was formulated in the previous chapter to ensure correct computation results. The first step was to code the DTA-UB formulation in Visual Studio $\mathrm{C}++$ and making sure that all bugs are removed and the model outputs are correct. Computational results are validated by running small networks. Small networks are used here to serve two purposes. Firstly, analyzing small networks helps to make sure that optimal solution is consistently obtained with change of parameter values and network mapping. To verify whether solution is optimal, computational results are compared with those obtained through manual solving using basic tools from Operations Research. Secondly, computational results are able to be listed individually and will provide readers an understanding of how they contribute to the final optimal solution, which will be humanly impossible to do for extremely large networks. The main analysis is to compare results from this model with that without lane reversals. As such, the next section presents a DTA-UB model without reversals and how it differs from DTA-UB with reversals. Subsequently, a brief summary regarding specifications of the operating system used to compute the model is given. Finally, examples of small networks are shown with detailed computational results and analysis.

\subsection{No Reversal Model}

No lane reversal DTA-UB (NRDTA-UB) shares the same core characteristics with the DTA-UB with reversals described in Chapter 3, with minimal differences. Just like DTA$\mathrm{UB}$, the time expanded network for NRDTA-UB is discretized into sets of discrete time 
points with $\delta$ representing the smallest time period in the formulation. The same travel time function is also used with free flow time $a$, taken as the minimum travel time on an arc and the maximum travel time is a function of the maximum flow possible. Weighted flow $x_{t}^{p, q}$ is taken as the variable used to calculate actual travel time of flow. The inverse nonlinear travel time constraint is used to find the equivalent linear constraints, which results in the formulation of the upper and lower bound of weighted flow. NRDTA-UB also shares the same flow balance constraint, just with absence of reversed flow $y_{p, q ; t, t+\tau}^{\prime}$. As there isn't any contraflow/lane reversals, so also there will not be any head-on collision of vehicles as a result of directional change of arcs in NRDTA-UB. As such, there is no need for collision prevention constraint (Constraint 3.17n) to be included here. Nevertheless, the result of not having collision prevention constraint is that the unique realization condition will not be achieved. Hence, the following constraints need to be included to ensure only one time expanded arc is realized for every static arc $(p, q)$,

$$
\sum_{\tau \in \Delta_{p, q}} z_{p, q ; t, t+\tau}=1, \quad \forall t \in \Gamma,(p, q) \in A
$$

Simultaneous route and departure (SRD) assumption is also incorporated in NRDTAUB, together with late departure penalties. The NRDTA-UB for emergency evacuation planning is formulated as follows,

$$
\begin{array}{ll}
\operatorname{Min} & \sum_{(p, q) \in A} \sum_{t \in \Gamma} \sum_{\tau \in \Delta_{p, q}} \tau \cdot y_{p, q ; t, t+\tau}+\sum_{i} \sum_{t} t \cdot w_{i, t} \\
\text { s.t. } & \sum_{t} w_{i, t}=h_{i}, \quad \forall i \in A \\
& \sum_{(p, s) \in A} \sum_{t} v_{p, s ; t}=h_{s} \\
& \sum_{q} \sum_{\tau} y_{p, q ; t, t+\tau}-\sum_{o} \sum_{\alpha} y_{o, p ; \alpha, \beta}-w_{i, t}=0, \\
\beta=t ; \forall t, \beta \in \Gamma ; \forall i, p \in A \\
\sum_{q} \sum_{\tau} y_{p, q ; t, t+\tau}-\sum_{o} \sum_{\alpha} y_{o, p ; \alpha, \beta}=0,
\end{array}
$$




$$
\begin{aligned}
& \beta=t ; \forall t, \beta \in \Gamma ; \forall i, p \in A \\
& \sum_{(p, s) \in A} \sum_{t} v_{p, s ; t}-\sum_{o} \sum_{\alpha} y_{o, p ; \alpha, \beta}=0, \quad \beta=t ; \forall t, \beta \in \Gamma ; \forall p \in A \\
& x_{t}^{p, q}=\sum_{\alpha} \sum_{\beta} y_{p, q ; \alpha, \beta}, \quad \forall t \in \Gamma ;(p, q) \in A ; \alpha \leq t, \beta \geq t \\
& x_{t}^{p, q} \geq \sum_{\tau \in \Delta_{p, q}} \phi_{p, q}^{-1}(\tau-\delta) z_{p, q ; t, t+\tau}, \quad \forall t \in \Gamma_{p, q} ;(p, q) \in A \\
& x_{t}^{p, q} \leq \sum_{\tau \in \Delta_{p, q}} \phi_{p, q}^{-1}(\tau) z_{p, q ; t, t+\tau}, \quad \forall t \in \Gamma_{p, q} ;(p, q) \in A \\
& y_{p, q ; t, t+\tau} \leq M_{p, q} z_{p, q ; t, t+\tau}, \quad \forall t \in \Gamma_{p, q} ; \tau \in \Delta_{p, q} ;(p, q) \in A \\
& \sum_{\tau \in \Delta_{p, q}} z_{p, q ; t, t+\tau}=1, \quad \forall t \in \Gamma,(p, q) \in A . \\
& v, w, x, y \geq 0 \\
& z_{p, q ; t, t+\tau} \in\{0,1\}, \quad \forall t \in \Gamma_{p, q} ; \tau \in \Delta_{p, q} ;(p, q) \in A
\end{aligned}
$$

The objective function is again split into two parts. The first part seeks to minimize travel time of flow, whereas the second part is the late departure penalty. Demand generation and flow absorbtion is shown in constraints $4.2 \mathrm{a}$ and $4.2 \mathrm{~b}$. The flow balance of time expanded network is represented by constraints $4.2 \mathrm{c}, 4.2 \mathrm{~d}$ and $4.2 \mathrm{e}$. Constraints $4.2 \mathrm{f}$ calculates the weighted flows. Constraints $4.2 \mathrm{~g}$ models the lower bound of regular flow travel time. Constraints $4.2 \mathrm{~h}$ models the upper bound of flow travel time. Constraint $4.2 \mathrm{i}$ and $4.2 \mathrm{j}$ ensures the unique travel time for each static arc at any time point. Also for convenience, $x$ denotes the decision vector composed of $x_{t}^{p, q}$. So do decision vectors $w, v$, and $y$. Definitions of indices, variables, and parameters can be found in Tables 3.1, 3.2, and 3.3 in Chapter 3.

\subsection{Computer Specifications and Software}

A Windows 7 Professional 64-bit operating system was used for testing the model with $3.10 \mathrm{GHz}$ of processing speed. Total usable random access memory (RAM) was 3.88 GB. Visual Studio C++ (2008) was used to code the formulation. IBM CPLEX was used to solve the formulation and obtain optimal solution. 


\subsection{Small Network: 2 Nodes}

As was mentioned earlier, in small scale networks, understanding of each flow's characteristics is simpler and model validation work more straight forward. Furthermore, when model is known to be correct, the same analysis can be applied to larger networks to prove the model's effectiveness. Figure 4.1 shows a simple two-node, two-lane network where node $p$ is the danger node and node $q$ is the safe node. Mapping details of this network is as follows:

- Number of nodes: 2

- Number of danger nodes: 1 (node: $p$ )

- Number of safe nodes: 1 (node: $q$ )

- Number of arcs: 2

- Number of time periods: 10

- Smallest time interval: 15 minutes

- Planning time horizon: 2.5 hours

- Demand: 30,000

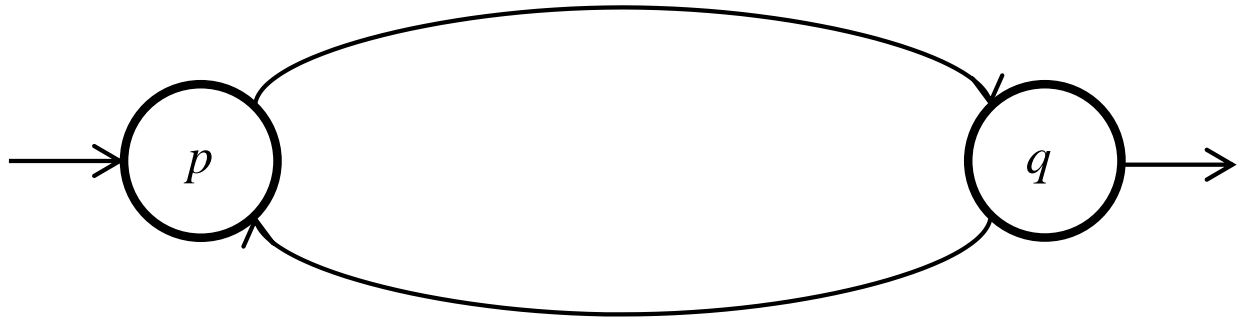

Figure 4.1: 2 node network.

The $\mathrm{C}++$ computer program for the models with reversals (DTA-UB) and without reversals (NRDTA-UB) are solved using CPLEX. Results for both models are shown in the sub-sections below when demand is fixed at 30,000. 


\subsubsection{Results for Model with Lane Reversal Starting Flow Output}

- Start-flow at node $p$ departing at time $0\left(w_{p, 0}\right): 20,000$

- Start-flow at node $p$ departing at time $1\left(w_{p, 1}\right): 10,000$

\section{Sink-Flow Output}

- Sink-flow absorbed at time $1\left(v_{p, q ; 1}\right): 20,000$

- Sink-flow absorbed at time $2\left(v_{p, q ; 2}\right): 10,000$

Intermediate Regular Flow Output

- Flow departing at time 0 and arriving at time $1\left(y_{p, q ; 0,1}\right): 10,000$

\section{Intermediate Reversed Flow Output}

- Reversed flow departing at time 0 and arriving at time $1\left(y_{q, p ; 0,1}^{\prime}\right): 10,000$

- Reversed flow departing at time 1 and arriving at time $2\left(y_{q, p ; 1,2}^{\prime}\right): 10,000$

Optimal Solution: 70,000

Computation Time: 0.078 seconds

CPLEX obtained the optimal solution based on the objective function (Equation 3.18) formulated in the model as follows,

$$
\tau \cdot y_{p, q ; 0,1}+\tau \cdot y_{q, p ; 0,1}^{\prime}+\tau \cdot y_{q, p ; 1,2}^{\prime}+t \cdot w_{0,0}+t \cdot w_{0,1}
$$

After plugging in the values, the following is obtained,

$$
10,000+10,000+10,000+20,000+2 \times 10,000=70,000
$$

Figure 4.2 shows the time expanded network with all flows that were realized where the solid lines represent regular flow, and the dotted lines represent reversed flow. As we can see, when the arc $(q, p ; 0,1)$ is reversed, the capacity of flow heading from node $p$ to node $q$ increases. 


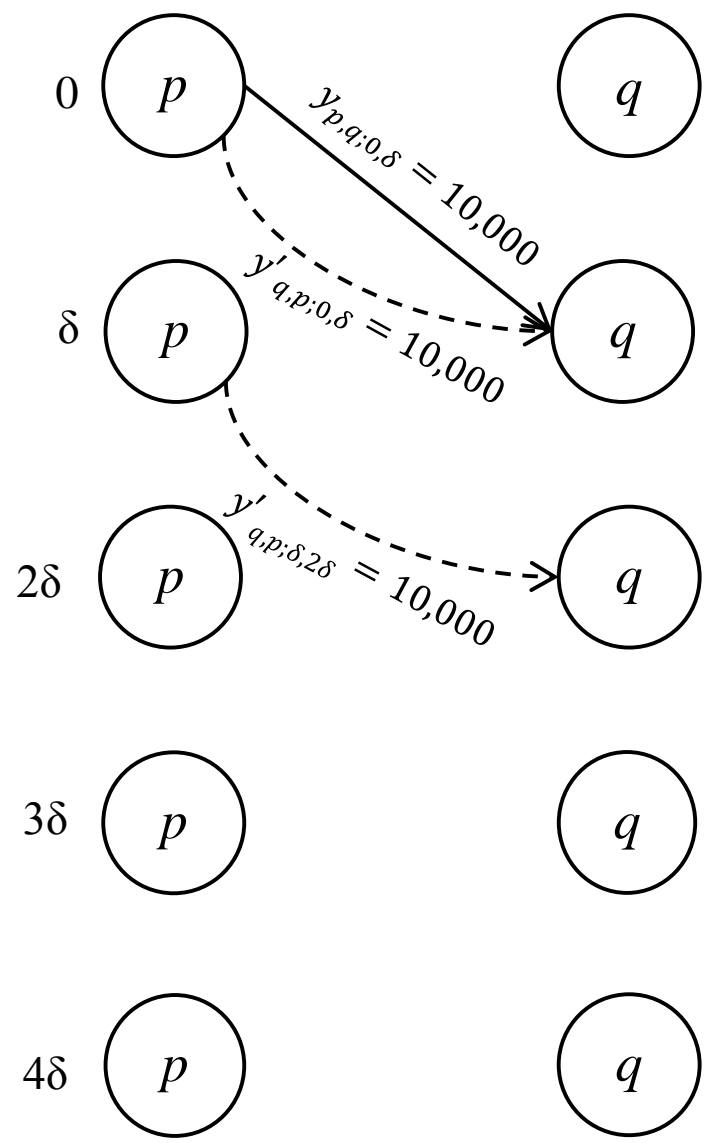

Figure 4.2: Solution for time expanded network with lane reversals.

\subsubsection{Results for Model without Lane Reversal}

\section{Starting Flow Output}

- Start-flow at node $p$ departing at time $0\left(w_{p, 0}\right): 10,000$

- Start-flow at node $p$ departing at time $1\left(w_{p, 1}\right)$ : 10,000

- Start-flow at node $p$ departing at time $2\left(w_{p, 2}\right): 10,000$

\section{Sink-Flow Output}

- Sink-flow absorbed at time $1\left(v_{p, q ; 1}\right)$ : 10,000

- Sink-flow absorbed at time $2\left(v_{p, q ; 2}\right): 10,000$

- Sink-flow absorbed at time $3\left(v_{p, q ; 3}\right): 10,000$

\section{Intermediate Regular Flow Output}


- Flow departing at time 0 and arriving at time $1\left(y_{p, q ; 0,1}\right): 10,000$

- Flow departing at time 1 and arriving at time $2\left(y_{p, q ; 1,2}\right): 10,000$

- Flow departing at time 2 and arriving at time $3\left(y_{p, q ; 2,3}\right): 10,000$

Optimal Solution: 90,000

Computation Time: 0.031 seconds

Just like the model with lane reversals, the objective function value obtained by CPLEX is as follows,

$$
\tau \cdot y_{p, q ; 0,1}+\tau \cdot y_{p, q ; 1,2}+\tau \cdot y_{p, q ; 2,3}+t \cdot w_{0,0}+t \cdot w_{0,1}+t \cdot w_{0,2}
$$

After plugging in the values, the following is obtained,

$$
10,000+10,000+10,000+10,000+2 \times 10,000+3 \times 10,000=90,000
$$

Figure 4.3 shows the time expanded network for no lane reversal model where the solid lines represent regular flow that is realized. In this case, since there isn't lane reversals to increase outbound capacities of arcs, all flows need to be distributed to depart at different times.

Table 4.1 summarizes the computational results of the 2-node model that is with lane reversals, and without lane reversals. With all inputs and parameters staying the same for both models, DTA-UB managed to produced better objective value by reversing lanes. This is due to the advantage of the capacity increasing of DTA-UB model. However, the computational time for NRDTA-UB is able to obtain optimal solution with quicker time. The simplicity of the model together with the absence of collision prevention restriction, as oppose to DTA-UB, are factors that contributes to the quicker solving time of NRDTAUB. 


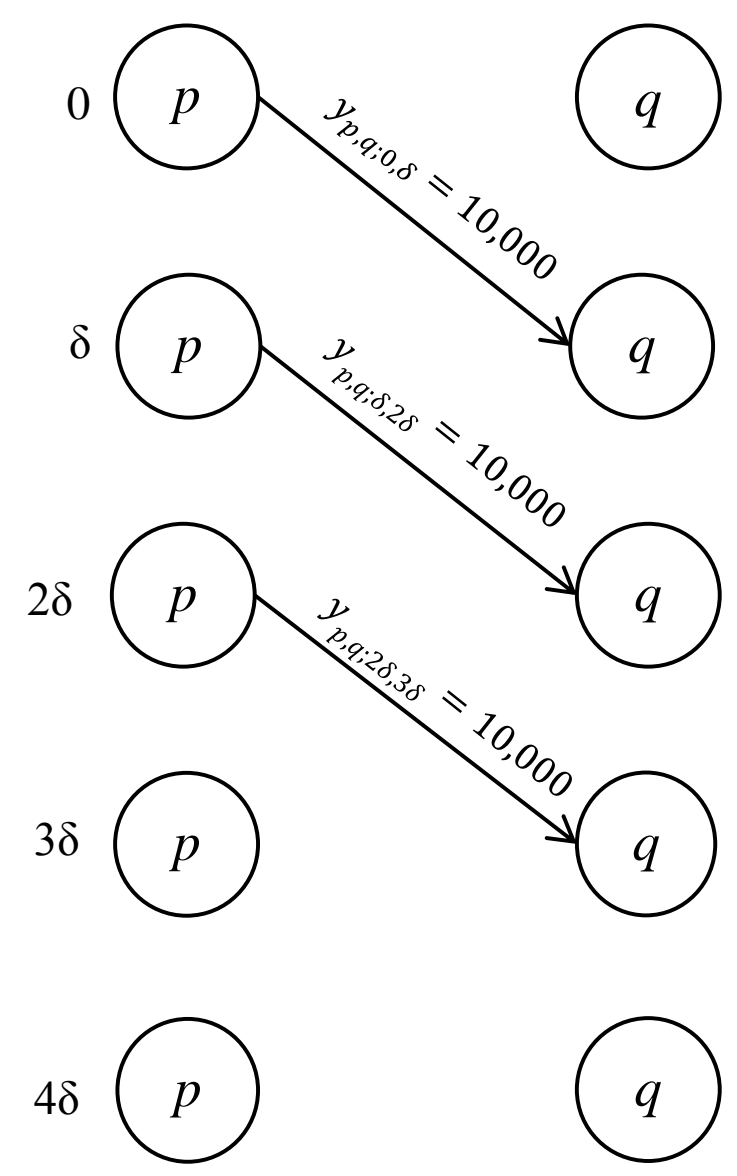

Figure 4.3: Solution for time expanded network without lane reversals.

\subsection{Small Network: 6 Nodes}

As can be seen from the above analysis, DTA-UB trumps NRDTA-UB for optimal results, but lack behind in computational time. To observe whether the same trend of results are consistent, a six-node network is analyzed here. Figure 4.4 shows the six-node network where there are two lanes/arcs in opposite directions connecting every O-D pairs, except for arcs linking to sink/safe node $u$. As such, all flows heading for safe node $u$ are also restricted so that no reversals are allowed. In this case, there are two nodes - node $p$ and $q$, that generate start-flow through assigning demand to each node respectively. Mapping details of this network is as follows:

- Number of nodes: 6

- Number of danger nodes: 5 (node: $p, q, r, s, t$ )

- Number of safe nodes: 1 (node: $u$ ) 
Table 4.1: 2-Node Model, DTA-UB vs. NRDTA-UB.

\begin{tabular}{|l||c|c|c|c|c|c|}
\hline \hline & Demand & $\begin{array}{l}\text { Time } \\
\text { Periods }\end{array}$ & Arcs & $\begin{array}{l}\text { Flow } \\
\text { Real- } \\
\text { ized }\end{array}$ & $\begin{array}{l}\text { Compu- } \\
\text { tation } \\
\text { Time }\end{array}$ & $\begin{array}{l}\text { Objec- } \\
\text { tive } \\
\text { Value }\end{array}$ \\
\hline \hline Model With Lane Reversal & 30,000 & 5 & 2 & $\begin{array}{l}y_{p, q ; 0,1} \\
y_{q, p ; 0,1}^{\prime} \\
y_{q, p ; 1,2}^{\prime}\end{array}$ & 0.078 & 70,000 \\
\hline Model Without Lane Reversal & 30,000 & 5 & 2 & $\begin{array}{l}y_{p, q ; 0,1} \\
y_{p, q ; 1,2} \\
y_{p, q ; 2,3}\end{array}$ & 0.031 & 90,000 \\
\hline
\end{tabular}

- Number of arcs: 14

- Number of time periods: 10

- Smallest time interval: 15 minutes

- Planning time horizon: 2.5 hours

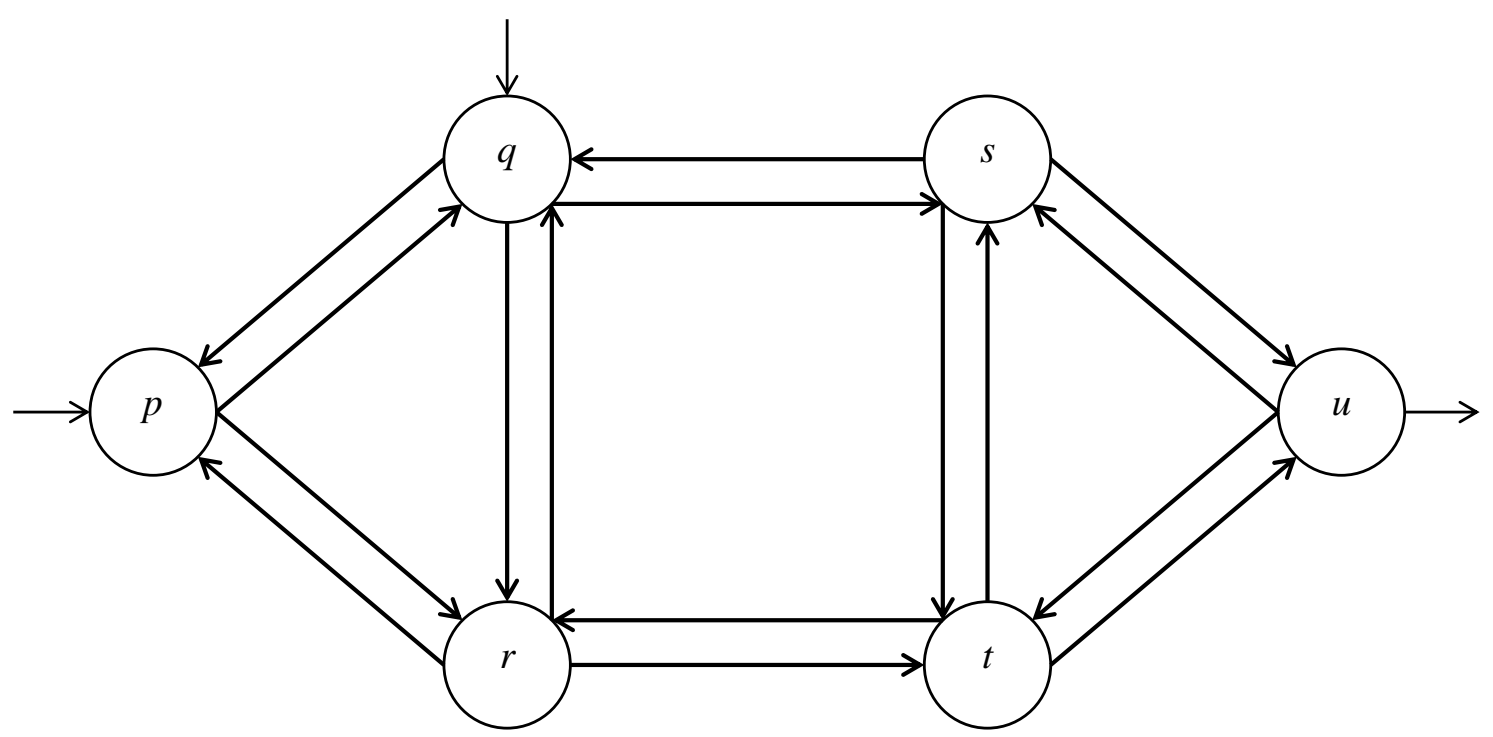

Figure 4.4: 6 node network.

Just like the two-node network, the $\mathrm{C}++$ computer program for the models with reversals (DTA-UB) and without reversals (NRDTA-UB) for six-node network are solved using CPLEX. Results for both models are shown in the sub-sections below when demand at node $p$ is fixed at 50,000 and demand at node $q$ is also fixed at 50,000. 


\subsubsection{Results for Model with Lane Reversal Starting Flow Output}

- Start-flow at node $p$ departing at time $0\left(w_{p, 0}\right): 40,000$

- Start-flow at node $p$ departing at time $1\left(w_{p, 1}\right): 10,000$

- Start-flow at node $q$ departing at time $0\left(w_{q, 0}\right): 20,000$

- Start-flow at node $q$ departing at time $1\left(w_{q, 1}\right): 10,000$

- Start-flow at node $q$ departing at time $2\left(w_{q, 2}\right): 10,000$

- Start-flow at node $q$ departing at time $3\left(w_{q, 3}\right): 10,000$

\section{Sink-Flow Output}

- Sink-flow absorbed for arc $(s, u)$ at time $1\left(v_{s, u ; 1}\right): 10,000$

- Sink-flow absorbed for arc $(s, u)$ at time $2\left(v_{s, u ; 2}\right): 20,000$

- Sink-flow absorbed for arc $(s, u)$ at time $3\left(v_{s, u ; 3}\right): 20,000$

- Sink-flow absorbed for arc $(s, u)$ at time $4\left(v_{s, u ; 4}\right)$ : 10,000

- Sink-flow absorbed for arc $(t, u)$ at time $2\left(v_{t, u ; 2}\right): 20,000$

- Sink-flow absorbed for $\operatorname{arc}(t, u)$ at time $3\left(v_{t, u: 3}\right): 20,000$

\section{Intermediate Regular Flow Output}

- Flow from $(p, q)$ departing at time 0 and arriving at time $1\left(y_{p, q ; 0,1}\right): 10,000$

- Flow from $(p, r)$ departing at time 0 and arriving at time $1\left(y_{p, r ; 0,1}\right): 10,000$

- Flow from $(q, r)$ departing at time 1 and arriving at time $2\left(y_{q, r ; 1,2}\right): 10,000$

- Flow from $(q, s)$ departing at time 0 and arriving at time $1\left(y_{q, s ; 0,1}\right): 10,000$

- Flow from $(q, s)$ departing at time 1 and arriving at time $2\left(y_{q, s ; 1,2}\right): 10,000$

- Flow from $(q, s)$ departing at time 2 and arriving at time $3\left(y_{q, s ; 2,3}\right): 10,000$ 
- Flow from $(q, s)$ departing at time 3 and arriving at time $4\left(y_{q, s ; 3,4}\right): 10,000$

- Flow from $(r, t)$ departing at time 1 and arriving at time $2\left(y_{r, t ; 1,2}\right): 10,000$

- Flow from $(r, t)$ departing at time 2 and arriving at time $3\left(y_{r, t ; 2,3}\right): 10,000$

\section{Intermediate Reversed Flow Output}

- Reversed flow from $(q, p)$ departing at time 0 and arriving at time $1\left(y_{q, p ; 0,1}^{\prime}\right): 10,000$

- Reversed flow from $(r, p)$ departing at time 0 and arriving at time $1\left(y_{r, p ; 0,1}^{\prime}\right): 10,000$

- Reversed flow from $(r, p)$ departing at time 1 and arriving at time $2\left(y_{r, p ; 1,2}^{\prime}\right): 10,000$

- Reversed flow from $(r, q)$ departing at time 0 and arriving at time $1\left(y_{r, q ; 0,1}^{\prime}\right): 10,000$

- Reversed flow from $(r, q)$ departing at time 1 and arriving at time $2\left(y_{r, q ; 1,2}^{\prime}\right): 10,000$

- Reversed flow from $(s, r)$ departing at time 1 and arriving at time $2\left(y_{s, r ; 1,2}^{\prime}\right): 10,000$

- Reversed flow from $(s, r)$ departing at time 2 and arriving at time $3\left(y_{s, r ; 2,3}^{\prime}\right): 10,000$

- Reversed flow from $(t, r)$ departing at time 1 and arriving at time $2\left(y_{t, r ; 1,2}^{\prime}\right): 10,000$

- Reversed flow from $(t, r)$ departing at time 1 and arriving at time $2\left(y_{t, r ; 2,3}^{\prime}\right): 10,000$

Optimal Solution: 350,000

Computation Time: 0.114 seconds

Optimal solution based on the objective function (Equation 3.18) is formulated as follows,

$$
\begin{aligned}
& \tau \cdot y_{p, q ; 0,1}+\tau \cdot y_{p, r ; 0,1}+\tau \cdot y_{q, r ; 1,2}+\tau \cdot y_{q, s ; 0,1}+\tau \cdot y_{q, s ; 1,2}+\tau \cdot y_{q, s ; 2,3}+\tau \cdot y_{q, s ; 3,4} \\
& +\tau \cdot y_{r, t ; 1,2}+\tau \cdot y_{r, t ; 2,3}+\tau \cdot y_{q, p ; 0,1}^{\prime}+\tau \cdot y_{r, p ; 0,1}^{\prime}+\tau \cdot y_{r, p ; 1,2}^{\prime}+\tau \cdot y_{r, q ; 0,1}^{\prime}+\tau \cdot y_{r, q ; 1,2}^{\prime} \\
& +\tau \cdot y_{s, r ; 1,2}^{\prime}+\tau \cdot y_{s, r ; 2,3}^{\prime}+\tau \cdot y_{t, r ; 1,2}^{\prime}+\tau \cdot y_{s, r ; 2,3}^{\prime} \\
& +t \cdot w_{p, 0}+t \cdot w_{p, 1}+t \cdot w_{q, 0}+t \cdot w_{q, 1}+t \cdot w_{q, 2}+t \cdot w_{q, 3}
\end{aligned}
$$

After plugging in the values, the following is obtained, 


$$
\begin{aligned}
& 9 \times 10,000+9 \times 10,000+40,000+2 \times 10,000 \\
& +20,000+2 \times 10,000+3 \times 10,000+4 \times 10,000=150,000
\end{aligned}
$$

\subsubsection{Results for Model without Lane Reversal Starting Flow Output}

- Start-flow at node $p$ departing at time $0\left(w_{p, 0}\right): 10,000$

- Start-flow at node $p$ departing at time $1\left(w_{p, 1}\right): 20,000$

- Start-flow at node $p$ departing at time $0\left(w_{q, 0}\right): 20,000$

- Start-flow at node $q$ departing at time $0\left(w_{q, 0}\right): 20,000$

- Start-flow at node $q$ departing at time $1\left(w_{q, 1}\right): 10,000$

- Start-flow at node $q$ departing at time $3\left(w_{q, 3}\right): 10,000$

- Start-flow at node $q$ departing at time $4\left(w_{q, 4}\right)$ : 10,000

\section{Sink-Flow Output}

- Sink-flow absorbed for arc $(s, u)$ at time $1\left(v_{s, u ; 1}\right)$ : 10,000

- Sink-flow absorbed for arc $(s, u)$ at time $2\left(v_{s, u ; 2}\right)$ : 10,000

- Sink-flow absorbed for arc $(s, u)$ at time $3\left(v_{s, u ; 3}\right)$ : 10,000

- Sink-flow absorbed for arc $(s, u)$ at time $4\left(v_{s, u ; 4}\right)$ : 10,000

- Sink-flow absorbed for arc $(s, u)$ at time $5\left(v_{s, u ; 5}\right)$ : 10,000

- Sink-flow absorbed for arc $(t, u)$ at time $2\left(v_{t, u ; 2}\right)$ : 10,000

- Sink-flow absorbed for arc $(t, u)$ at time $3\left(v_{t, u ; 3}\right)$ : 10,000

- Sink-flow absorbed for arc $(t, u)$ at time $4\left(v_{t, u ; 4}\right)$ : 10,000

- Sink-flow absorbed for arc $(t, u)$ at time $5\left(v_{t, u ; 5}\right)$ : 10,000 
- Sink-flow absorbed for arc $(t, u)$ at time $6\left(v_{t, u ; 6}\right): 10,000$

\section{Intermediate Regular Flow Output}

- Flow from $(p, q)$ departing at time 1 and arriving at time $2\left(y_{p, q ; 1,2}\right): 10,000$

- Flow from $(p, q)$ departing at time 2 and arriving at time $3\left(y_{p, q ; 2,3}\right): 10,000$

- Flow from $(p, r)$ departing at time 0 and arriving at time $1\left(y_{p, r ; 0,1}\right): 10,000$

- Flow from $(p, r)$ departing at time 1 and arriving at time $2\left(y_{p, r ; 1,2}\right): 10,000$

- Flow from $(p, r)$ departing at time 2 and arriving at time $3\left(y_{p, r ; 2,3}\right): 10,000$

- Flow from $(p, r)$ departing at time 4 and arriving at time $5\left(y_{p, r ; 4,5}\right): 10,000$

- Flow from $(q, r)$ departing at time 0 and arriving at time $1\left(y_{q, r ; 0,1}\right): 10,000$

- Flow from $(q, r)$ departing at time 2 and arriving at time $3\left(y_{q, r ; 2,3}\right): 10,000$

- Flow from $(q, r)$ departing at time 3 and arriving at time $4\left(y_{q, r ; 3,4}\right): 10,000$

- Flow from $(q, s)$ departing at time 0 and arriving at time $1\left(y_{q, s ; 0,1}\right): 10,000$

- Flow from $(q, s)$ departing at time 1 and arriving at time $2\left(y_{q, s ; 1,2}\right): 10,000$

- Flow from $(q, s)$ departing at time 2 and arriving at time $3\left(y_{q, s ; 2,3}\right): 10,000$

- Flow from $(q, s)$ departing at time 3 and arriving at time $4\left(y_{q, s ; 3,4}\right): 10,000$

- Flow from $(q, s)$ departing at time 4 and arriving at time $5\left(y_{q, s ; 4,5}\right): 10,000$

- Flow from $(r, p)$ departing at time 3 and arriving at time $4\left(y_{r, p ; 3,4}\right): 10,000$

- Flow from $(r, q)$ departing at time 1 and arriving at time $2\left(y_{r, q ; 1,2}\right): 10,000$

- Flow from $(r, t)$ departing at time 1 and arriving at time $2\left(y_{r, t ; 1,2}\right): 10,000$

- Flow from $(r, t)$ departing at time 2 and arriving at time $3\left(y_{r, t ; 2,3}\right): 10,000$

- Flow from $(r, t)$ departing at time 3 and arriving at time $4\left(y_{r, t ; 3,4}\right): 10,000$

- Flow from $(r, t)$ departing at time 4 and arriving at time $5\left(y_{r, t ; 4,5}\right): 10,000$ 
- Flow from $(r, t)$ departing at time 5 and arriving at time $6\left(y_{r, t ; 5,6}\right): 10,000$

Optimal Solution: 410,000

Computation Time: 0.068 seconds

Just like the model with lane reversals, the objective function value obtained by CPLEX is as follows,

$$
\begin{aligned}
& \tau \cdot y_{p, q ; 1,2}+\tau \cdot y_{p, q ; 2,3}+\tau \cdot y_{p, r ; 0,1}+\tau \cdot y_{p, r ; 1,2}+\tau \cdot y_{p, r ; 2,3}+\tau \cdot y_{p, r ; 4,5}+\tau \cdot y_{q, r ; 0,1}+\tau \cdot y_{q, r ; 2,3} \\
& +\tau \cdot y_{q, r ; 3,4}+\tau \cdot y_{q, s ; 0,1}+\tau \cdot y_{q, s ; 1,2}+\tau \cdot y_{q, s ; 2,3}+\tau \cdot y_{q, s ; 3,4}+\tau \cdot y_{q, s ; 4,5}+\tau \cdot y_{r, p ; 3,4}+\tau \cdot y_{r, q ; 1,2} \\
& +\tau \cdot y_{r, t ; 1,2}+\tau \cdot y_{r, t ; 2,3}+\tau \cdot y_{r, t ; 3,4}+\tau \cdot y_{r, t ; 4,5}+\tau \cdot y_{r, t ; 5,6} \\
& +t \cdot w_{p, 0}+t \cdot w_{p, 1}+t \cdot w_{q, 0}+t \cdot w_{q, 1}+t \cdot w_{q, 3}+t \cdot w_{q, 4}
\end{aligned}
$$

After plugging in the values, the following is obtained,

$$
\begin{aligned}
& 21 \times 10,000+10,000+2 \times 20,000+20,000+20,000+2 \times 10,000 \\
& +4 \times 10,000+5 \times 10,000=410,000
\end{aligned}
$$

Table 4.2 summarizes the computational results of the 6-node model that is with lane reversals, and without lane reversals.

Table 4.2: 6-Node Model, DTA-UB vs. NRDTA-UB.

\begin{tabular}{|l||c|c|c|c|c|}
\hline \hline & Demand & $\begin{array}{l}\text { Time Pe- } \\
\text { riods }\end{array}$ & Arcs & $\begin{array}{l}\text { Compu- } \\
\text { tation } \\
\text { Time }\end{array}$ & $\begin{array}{l}\text { Objec- } \\
\text { tive Value }\end{array}$ \\
\hline \hline Model With Lane Reversal & 100,000 & 5 & 14 & 0.114 & 350,000 \\
\hline Model Without Lane Reversal & 100,000 & 5 & 14 & 0.068 & 410,000 \\
\hline
\end{tabular}

Analysis of the six-node network again shows the same characteristics as the two-node network: DTA-UB produces better objective value by reversing lanes. Computational time for NRDTA-UB to obtain optimal solution again was quicker. 


\section{Chapter 5}

\section{Numerical Results}

Detailed comparison of computational results and optimal routing between the networks with lane reversals and without lane reversals were given in Chapter 4. However, for networks that are much larger, it is near impossible to observe flow on every arc, departure time of every user, and other minute factors. This is due to the fact that complexity of the mathematical model increases exponentially for larger networks as constraints, model size, and number of variables increase in turn. Hence, instead of focusing on the minute details, the trend of optimal values and computational time as demand increases for DTA-UB and NRDTA-UB are the main focus in this chapter. The following sections present Sioux Falls and Anaheim Network and their numerical results. A graphical representation of the results output are also provided. Both networks including their trip tables and other relevant information were taken from the webpage managed by Hillel Bar-Gera [75]. It is a webpage that compiles transportation network test problems contributed by various people in academia for academic research purposes.

\subsection{Sioux Falls Network}

Like all transportation network modeling, the amount nodes and lanes/arcs used in the model determine the level of detail and complexity of the problem. The more nodes and arcs are represented, the more detailed and complex the problem. Figure 5.1 produced by Hai Yang and Meng Qiang from Hong Kong University of Science and Technology, shows a simple 25 nodes and 80 arcs layout of the Sioux Falls Network. The safe zones were assumed to be at the bottom. As such, users are intended to head towards the safe nodes, 
which are assigned as nodes 13, 24, 21, and 20. Additional information of the network model are as follows:

- Number of nodes: 25

- Number of danger nodes: 20

- Number of safe nodes: 4

- Number of arcs: 80

- Planning time horizon: 10 hours

\subsubsection{Smallest Time Interval: 15 Minutes}

This section considers networks with the smallest time interval assigned as 15 minutes. With the planning time horizon fixed at 10 hours, the number of time periods depend upon the smallest time interval. The values of both attributes are listed as follows:

- Number of time periods: 40

- Smallest time interval: 15 minutes

Table 5.1 records the computational results of the Sioux Falls Network that has a smallest time interval of 15 minutes. The first two columns are the lower and upper bound of start-flow of each individual nodes. The third column is the sum of the entire demand within the network. The solving time and objective value for both DTA-UB and NRDTA-UB are recorded and the percentage difference of results between both networks are calculated in the last two columns. Figures 5.2 and 5.3 present the graphical view of objective value and solving time of both DTA-UB and NRDTA-UB models as demand increases.

From table 5.1, it can be seen that the difference of objective value started off almost equal between DTA-UB and NRDTA-UB. As demand increases, the DTA-UB model managed to maintain a lower objective value than NRDTA-UB. The difference of objective value between the two models also rapidly increases as demand increases. NRDTA-UB generated infeasible solution at 1,500,000 demand, as oppose to DTA-UB which only obtain infeasibility at 2,900,000 demand. Hence, the amount of demand DTA-UB was 


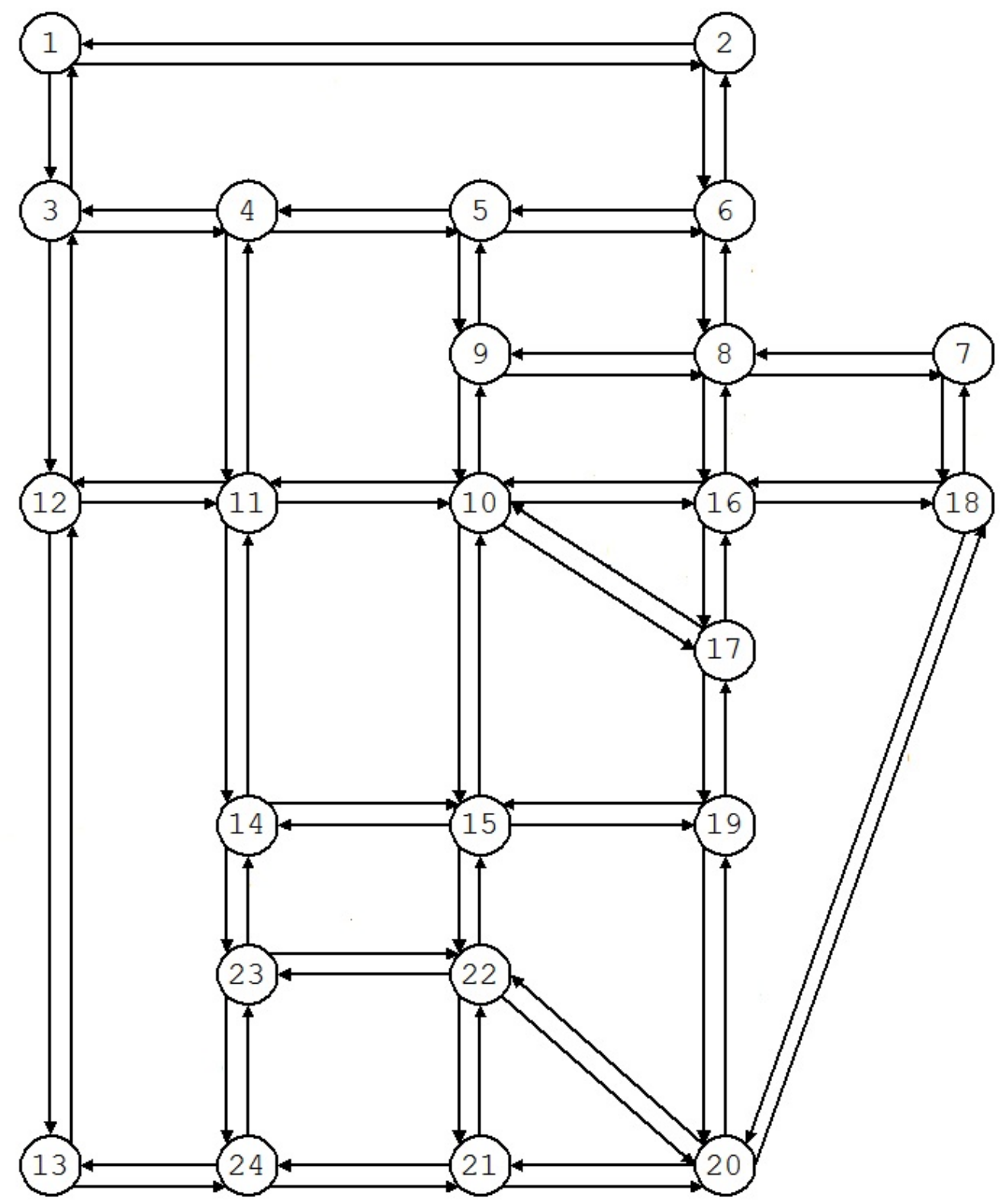

Figure 5.1: Sioux Falls Network [Yang and Meng].

able to optimize far exceeded that of NRDTA-UB. From figure 5.2, it can be seen that although objective value generated from both models increases exponentially as demand increases, DTA-UB maintained a better optimal solution at every demand point. It can also be seen that NRDTA-UB obtained infeasibility at approximately half the demand that of DTA-UB. Furthermore, the solving time of both DTA-UB and NRDTA-UB follows a linearly increasing trend as can be seen in Figure 5.3. Although the network with lane reversals has longer solving time at all demand points, it appears that both models' solving 
time increases in an almost equal ratio in this case.

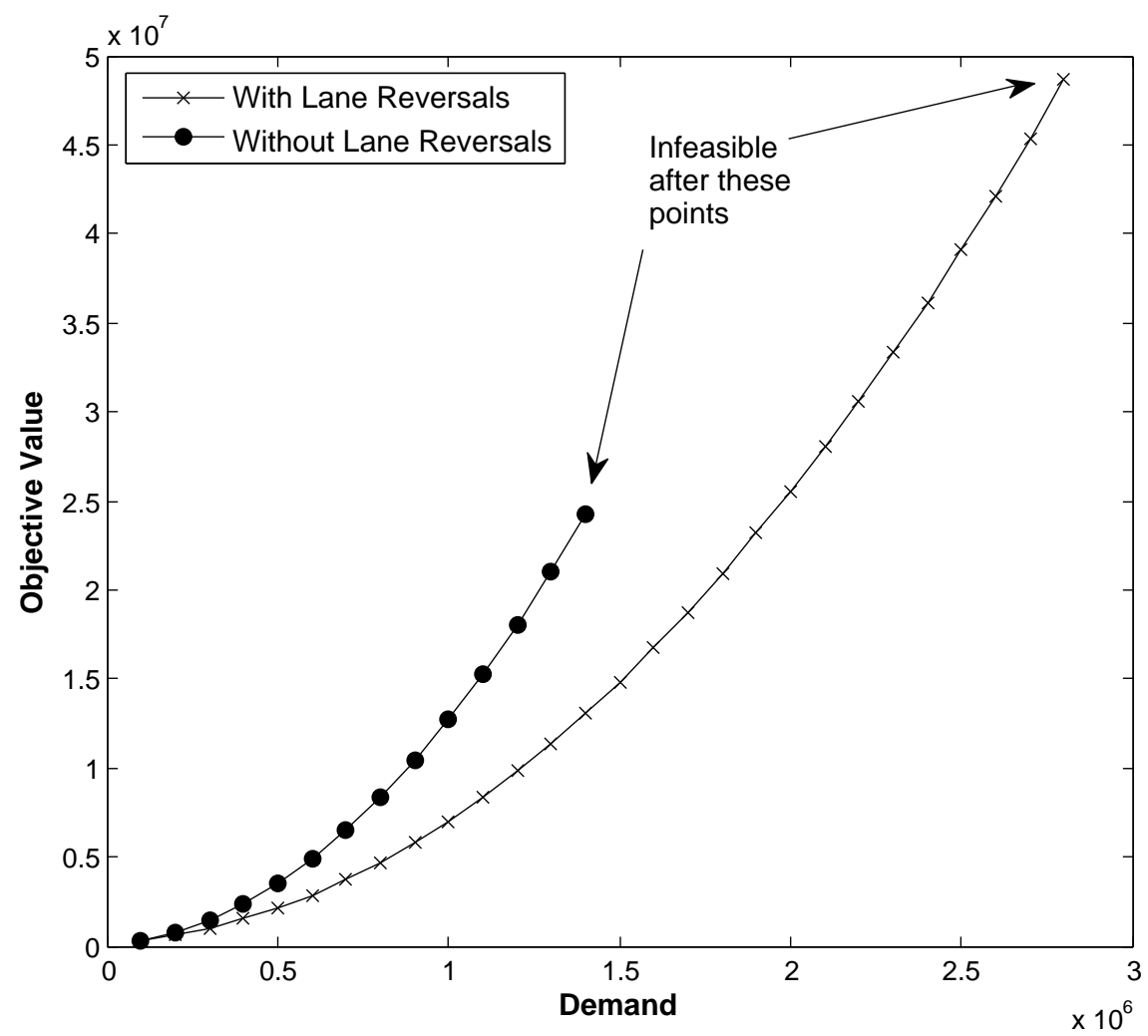

Figure 5.2: Sioux Falls Network Objective Value vs. Demand, Smallest Time Interval: 15 Minutes.

\subsubsection{Smallest Time Interval: 30 Minutes}

This section considers networks with the smallest time interval assigned as 30 minutes. With the planning time horizon fixed at 10 hours, the values of both attributes are listed as follows:

- Number of time periods: 20

- Smallest time interval: 30 minutes

Table 5.2 records the computational results of Sioux Falls Network that has a smallest time interval of 30 minutes. The format of the results presented is identical to Table 5.1. Figure 5.4 and 5.5 presents the graphical view of the objective value and solving time of both DTA-UB and NRDTA-UB models as demand increases. 


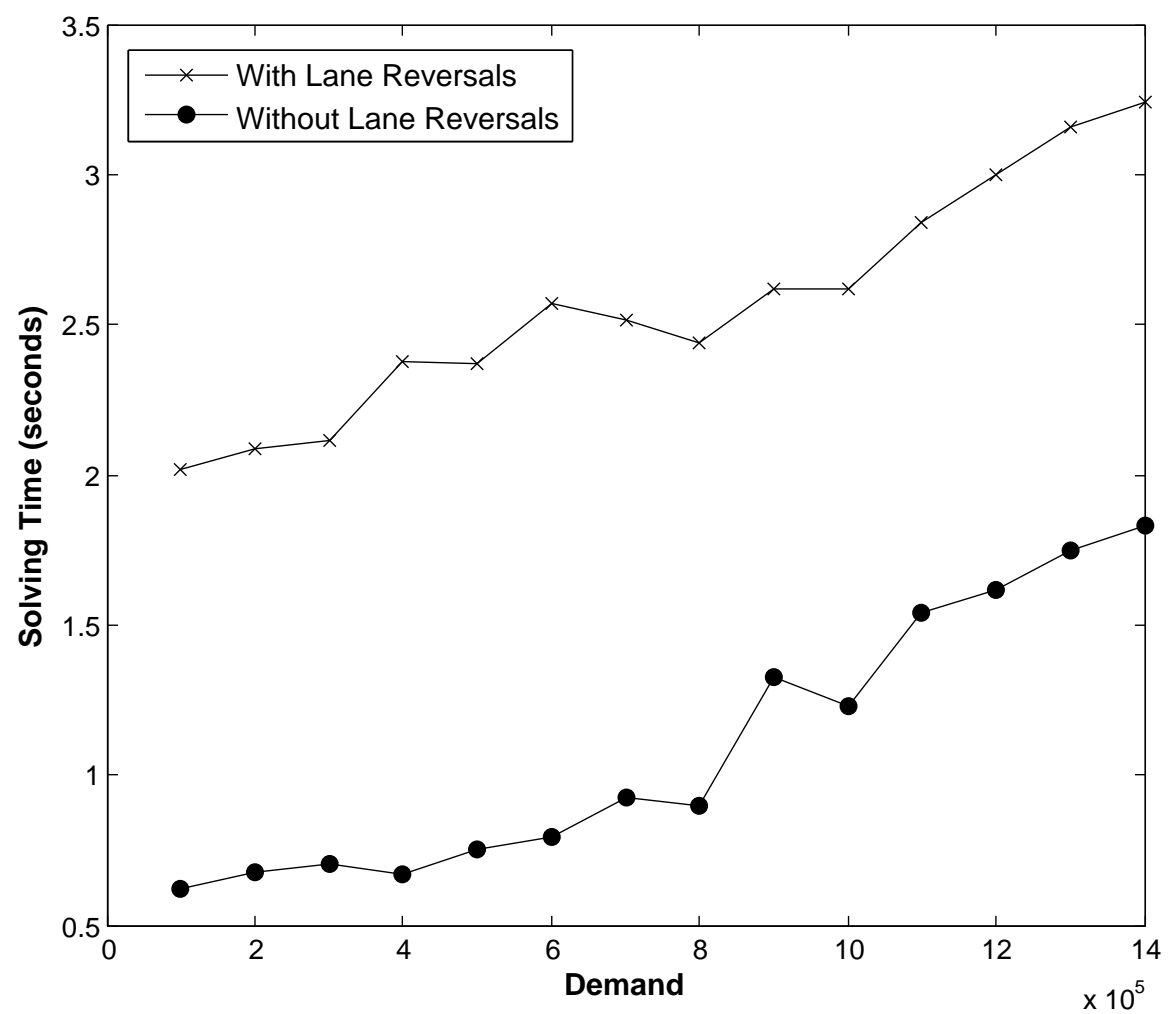

Figure 5.3: Sioux Falls Network Solving Time vs. Demand, Smallest Time Interval: 15 Minutes.

The results from the networks with smallest time interval of 30 minutes carry the same trend as that with smallest time interval of 15 minutes. Table 5.2 shows that the difference in objective value of DTA-UB and NRDTA-UB again started off almost equal, and rapidly increased as demand increased with DTA-UB maintaining a lower objective value throughout the process. Also, NRDTA-UB obtained infeasibility at 80,000 demand whereas DTA-UB managed to continue to 1,500,000 demand before obtaining infeasibility. Figure 5.4 further illustrate DTA-UB being able to obtain better optimal solution at every demand point. Solving time of both networks also follows a linearly increasing trend with NRDTA-UB being able to solve for optimal solution quicker, as shown in Figure 5.5.

\subsection{Anaheim Network}

Anaheim network, first provided by Jeff Ban and Ray Jayakrishnan, is a much larger and complex network compared to the Sioux Falls network due to the amount of nodes 


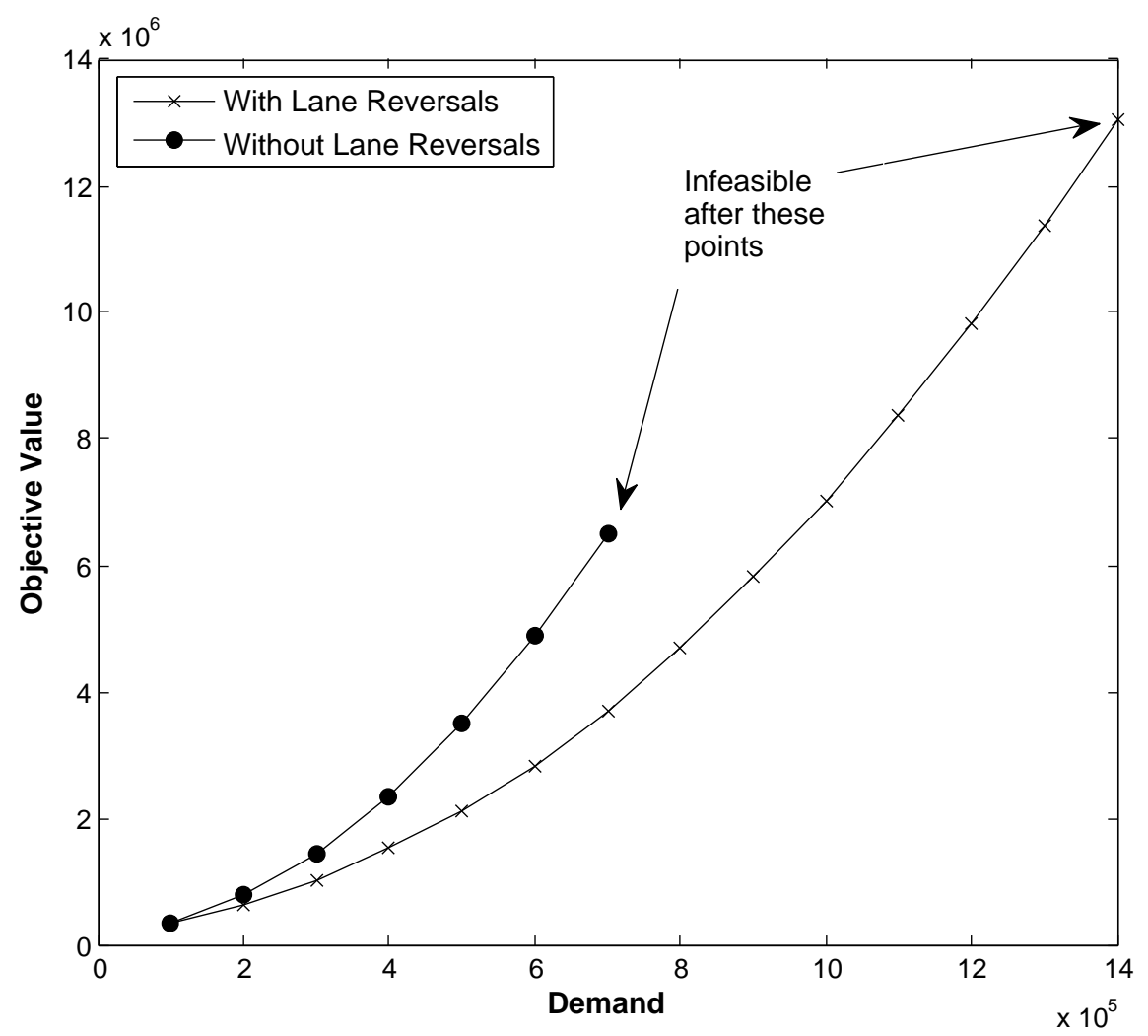

Figure 5.4: Sioux Falls Network Objective Value vs. Demand, Smallest Time Interval: 15 Minutes.

and arcs it has. It is difficult to provide a graphical representation of the Anaheim network that shows in detail all nodes and connecting arcs. Figure 5.6 [MapQuest.com] however shows the map of the layout of Anaheim. Additional details when formulating Anaheim network are as follows:

- Number of nodes: 417

- Number of danger nodes: 400

- Number of safe nodes: 16

- Number of arcs: 930

- Single-directional OD pair: 346

- 2-directional OD pair: 284

- Number of time periods: 48 


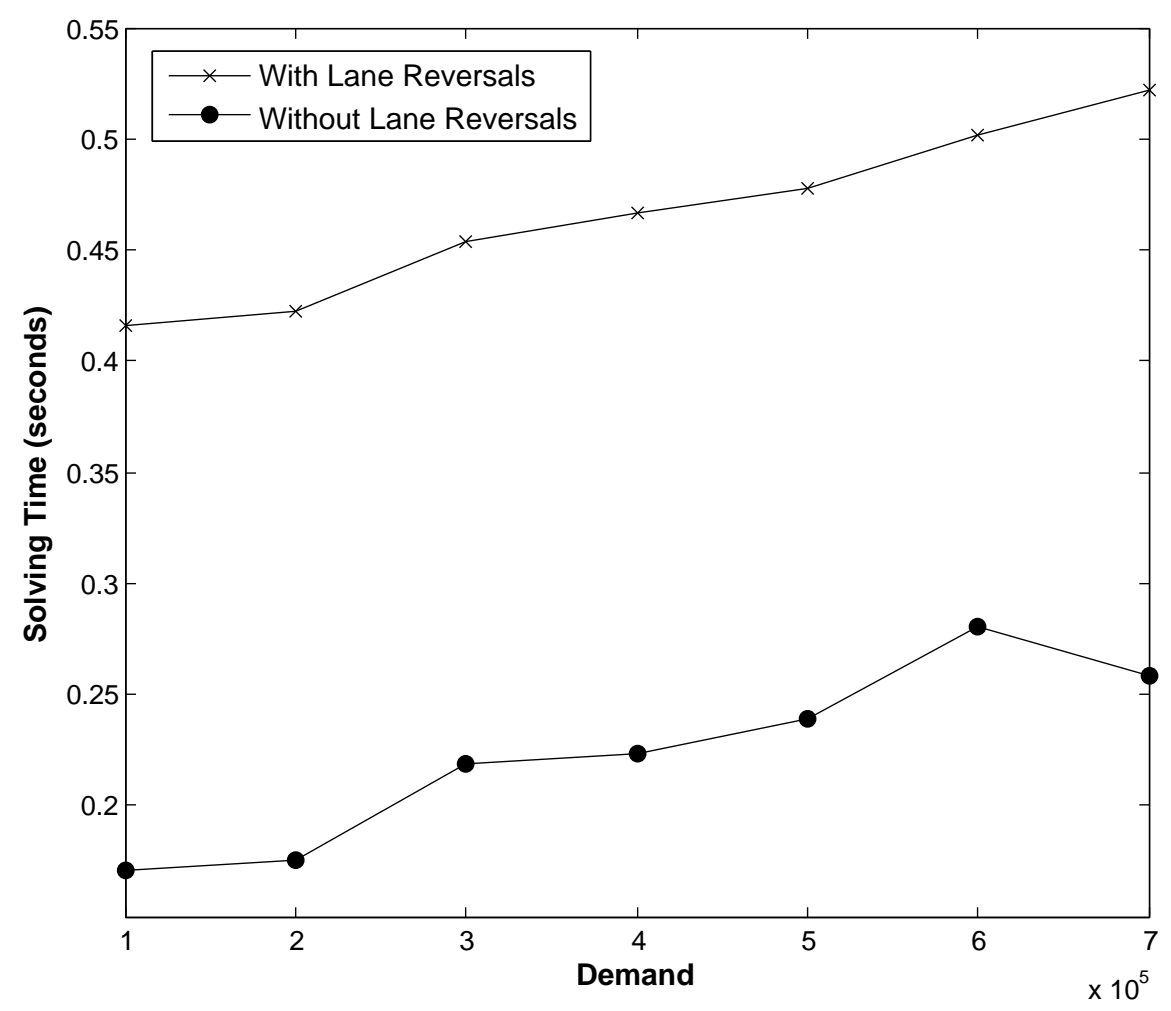

Figure 5.5: Sioux Falls Network Solving Time vs. Demand, Smallest Time Interval: 15 Minutes.

- Smallest time interval: 15 minutes

- Planning time horizon: 12 hours

Table 5.3 records the computational results and Figure 5.7 presents the graphical view of the objective value of both DTA-UB and NRDTA-UB. Like the Sioux Falls network, DTA-UB obtained better optimal solution throughout the entire process. Also, NRDTAUB obtained infeasibility at 12,500,000 demand whereas DTA-UB managed to continue to 25, 500, 000 before obtaining infeasibility. Solving time for the network with lane reversals started off with a linearly increasing trend, however after a certain demand point, it suddenly jumps to a much greater value, and retains that range for the next few demand points. Figure 5.8 illustrates this example.

Notice that when modeling the Anaheim network above, there exist 284 2-directional OD pairs and 346 single-directional OD pairs. In other words, only 284 OD pairs have 2 arcs connecting the two nodes and 346 OD pairs have 1 arc connecting the nodes. This is a realistic representation as not all roads connecting 2 separate towns/cities/nodes are 


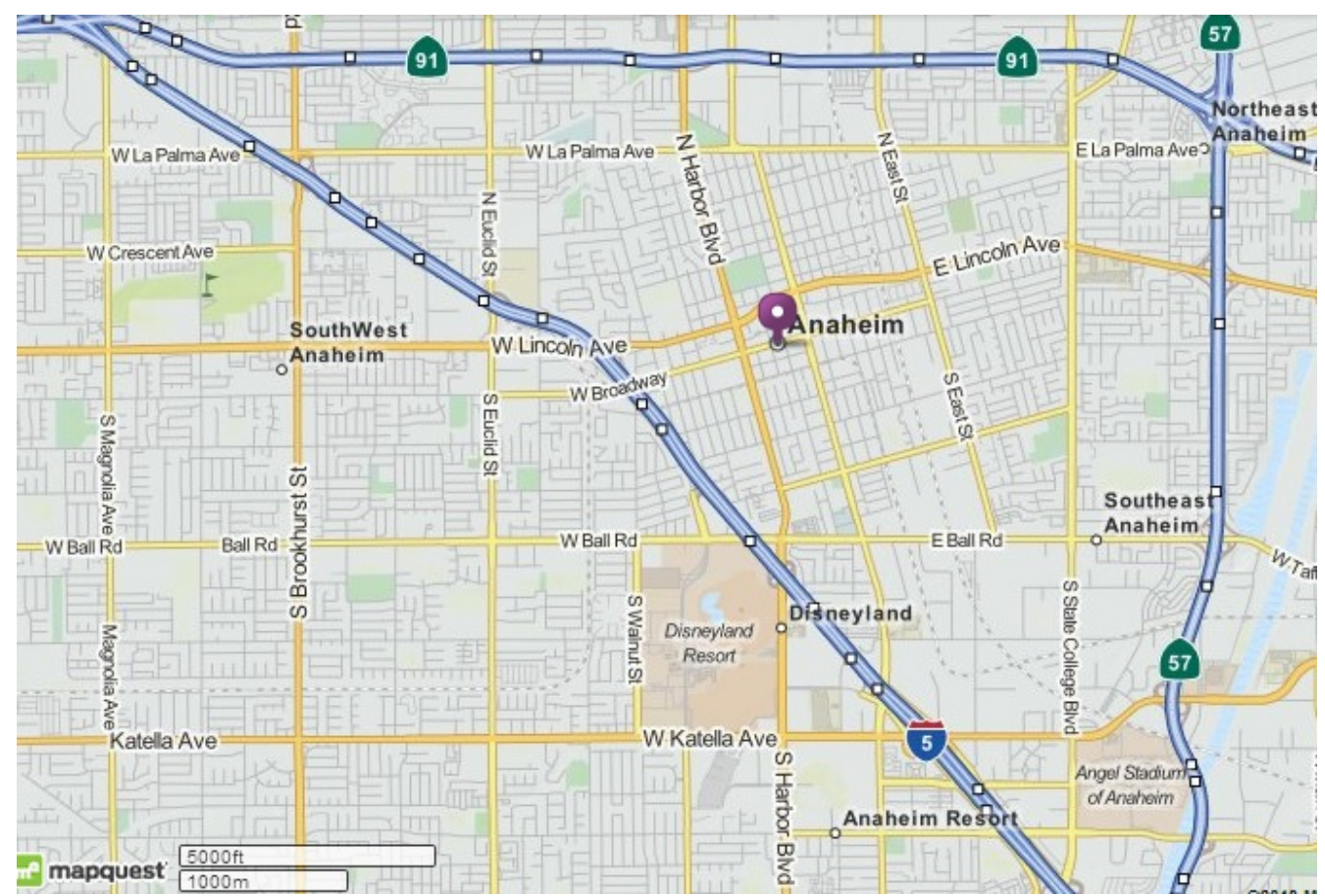

Figure 5.6: Map of Anaheim.

2-directional, there may exist only one-way roads leading from one node to the other. However, in this research, a network with 2-directional arcs on every OD pair needs to be modeled to compare its computational results with the original network layout. To do this, a Macro-enabled Microsoft Excel code was written so that for every arc connecting 2-nodes, there exist another identical arc in the reversed direction. With this, details of the new network are changed to as follows:

- Number of arcs: 1260

- Single-directional OD pair: 0

- 2-directional OD pair: 630

The number of danger nodes, safe nodes, time periods, smallest time interval, and planning time horizon remains the same as before. Table 5.4 records the computational results of the new Anaheim network. Figure 5.10 compares the objective value of the complete 2-directional OD pairs and partial 2-directional OD pairs of Anaheim network. It can be seen that although the objective value of both networks started off almost equal, the complete 2-directional OD pairs network eventually achieved better optimal value than 


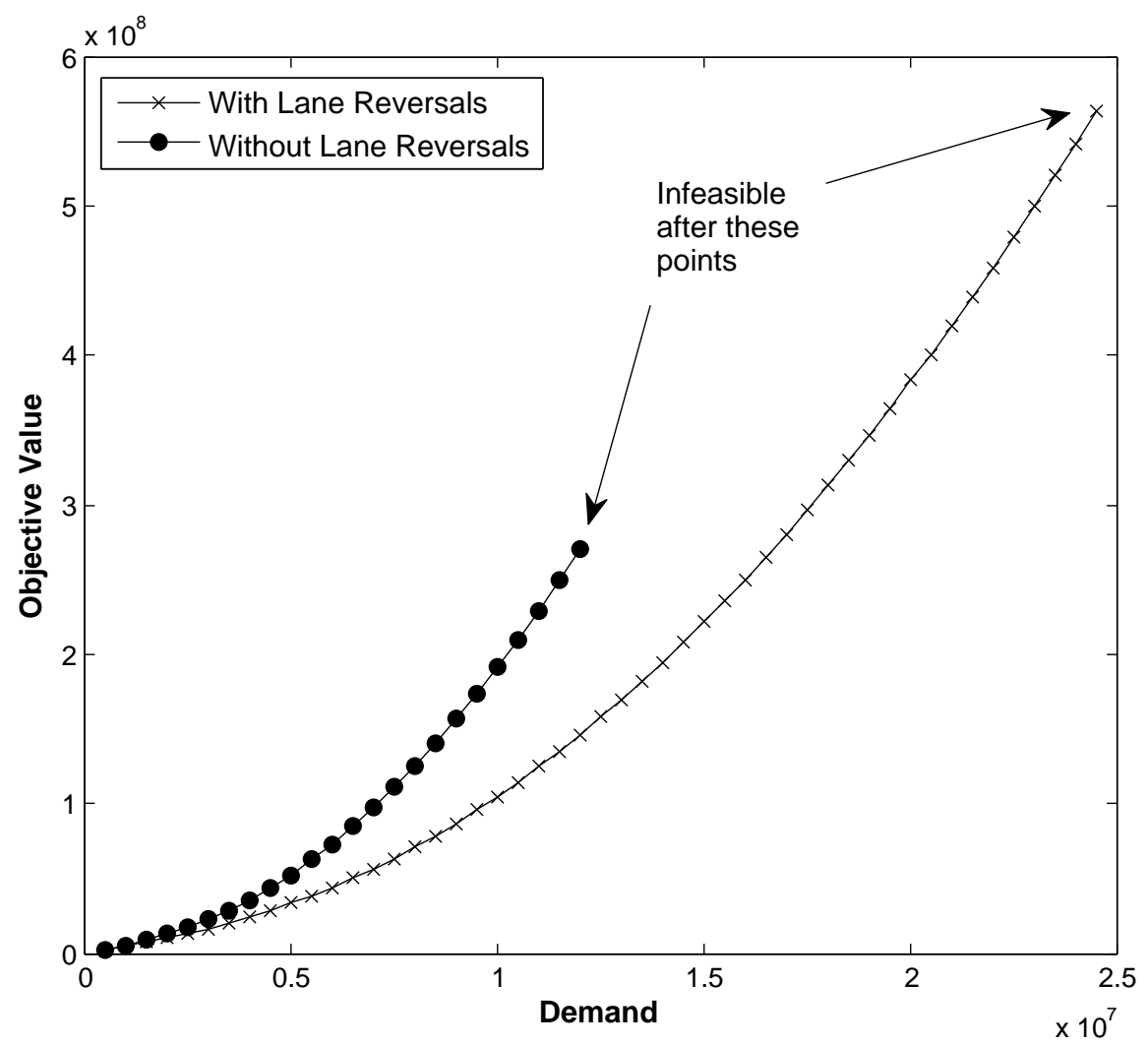

Figure 5.7: Anaheim Network Objective Value vs. Demand.

the partial network. Also, complete 2-directional OD pairs network was able to optimize up to 32,500,000 demand before obtaining infeasibility. This is 7,000,000 more than partial 2-directional OD pairs network. Figure 5.10 presents the solving time of partial and complete 2-directional OD pairs networks. Both networks had similar solving time at the beginning, but eventually complete 2-directional OD pairs networks surpassed the partial network and their solving time differences continued to increase as demand increased.

\subsection{Chapter Summary}

Based on the empirical results shown from the sections above, the following conclusions can be made:

- Network with lane reversals is able to obtain better objective value than network without lane reversals. 


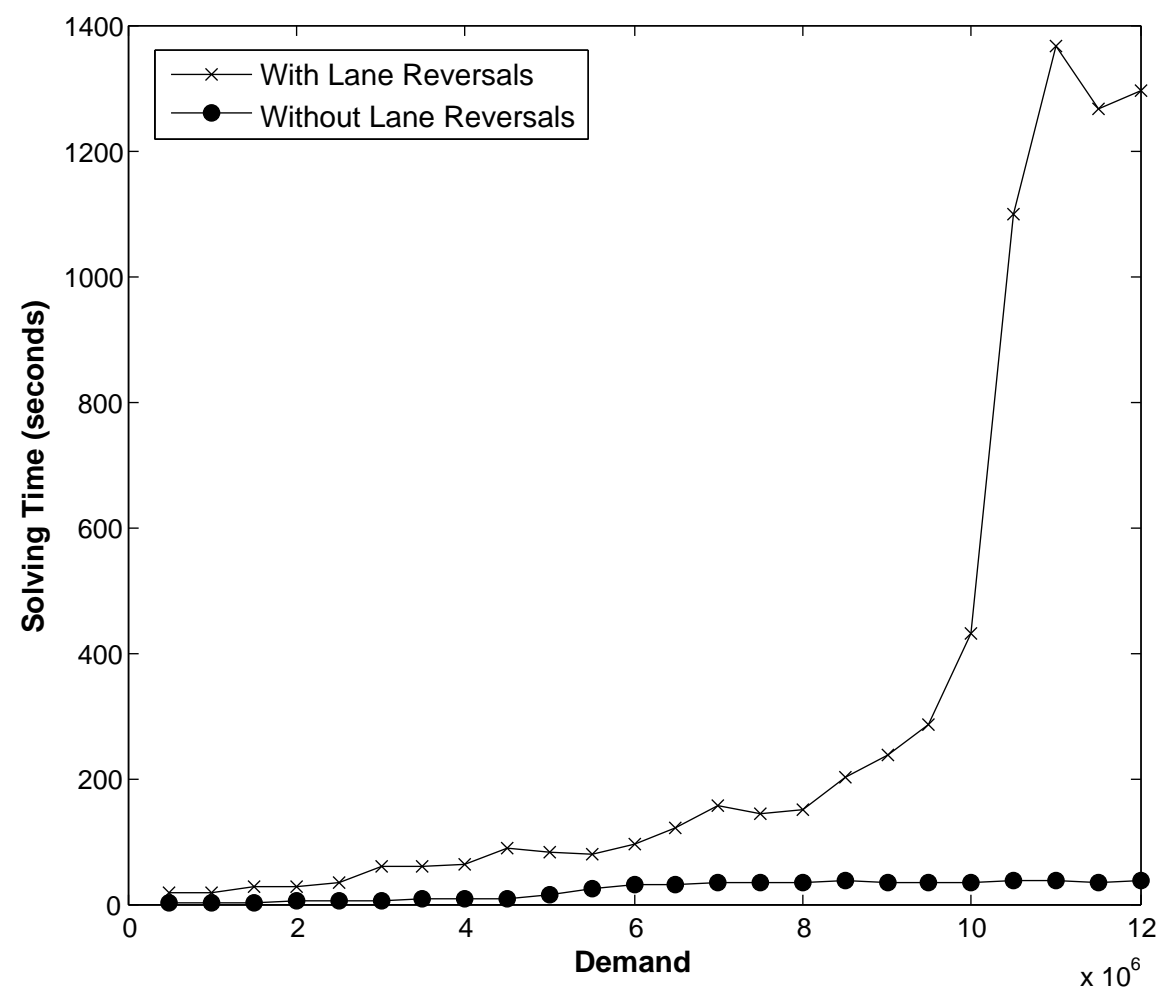

Figure 5.8: Anaheim Network Solving Time vs. Demand.

- Network with lane reversals is able to optimize almost double the demand of network without reversals.

- Solving time of both networks with and without reversals linearly increases at the beginning with demand.

- Solving time of network with lane reversals is consistently greater than that without lane reversals. At a certain demand point, solving time breaks from its linear path and jumps to a much higher value.

- Complete 2-directional OD pairs network obtains better objective value and is also able to optimize greater demand than partial 2-directional OD pairs network. 


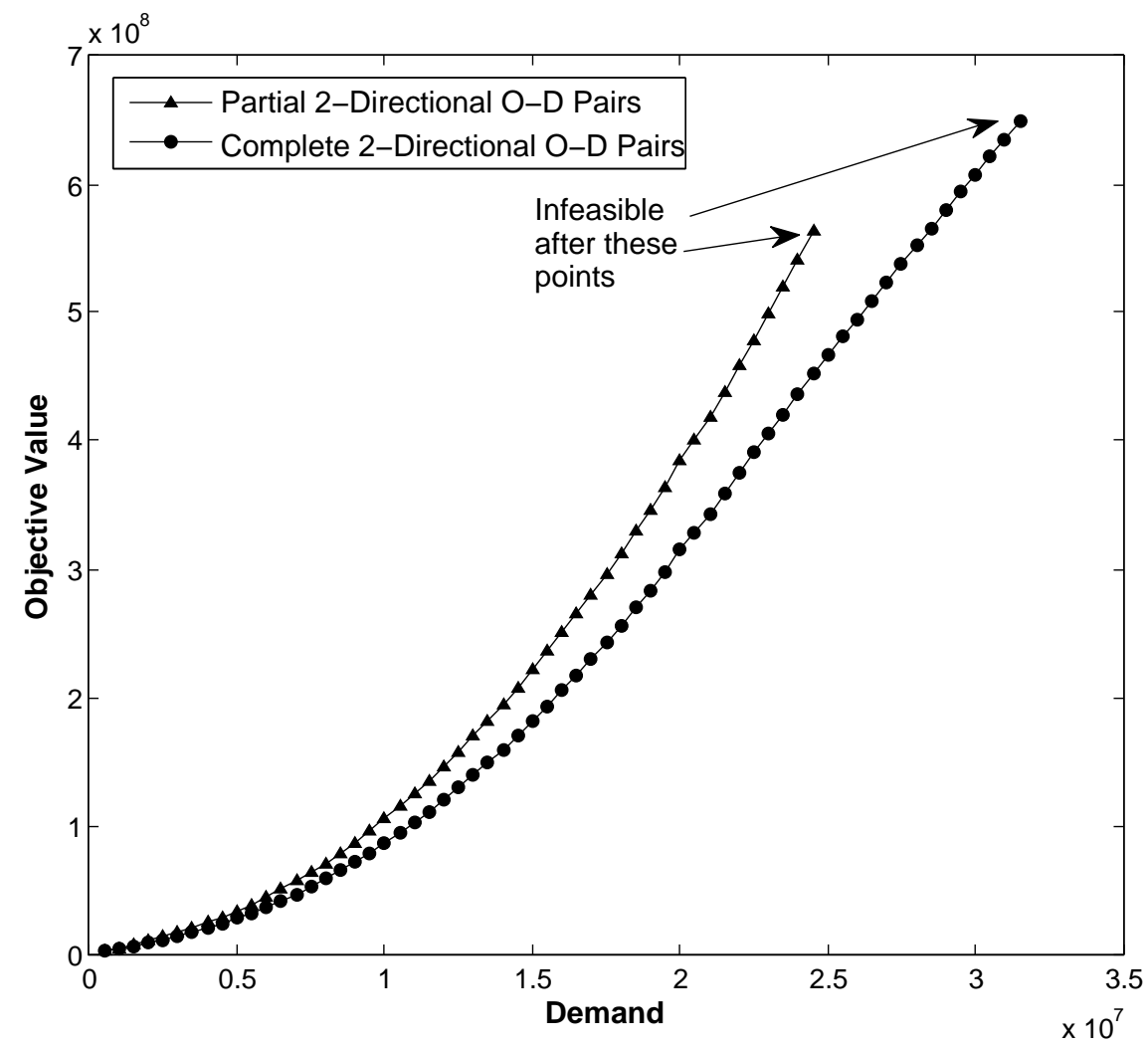

Figure 5.9: Partial and Complete 2-Directional O-D Pairs Objective Value vs. Demand.

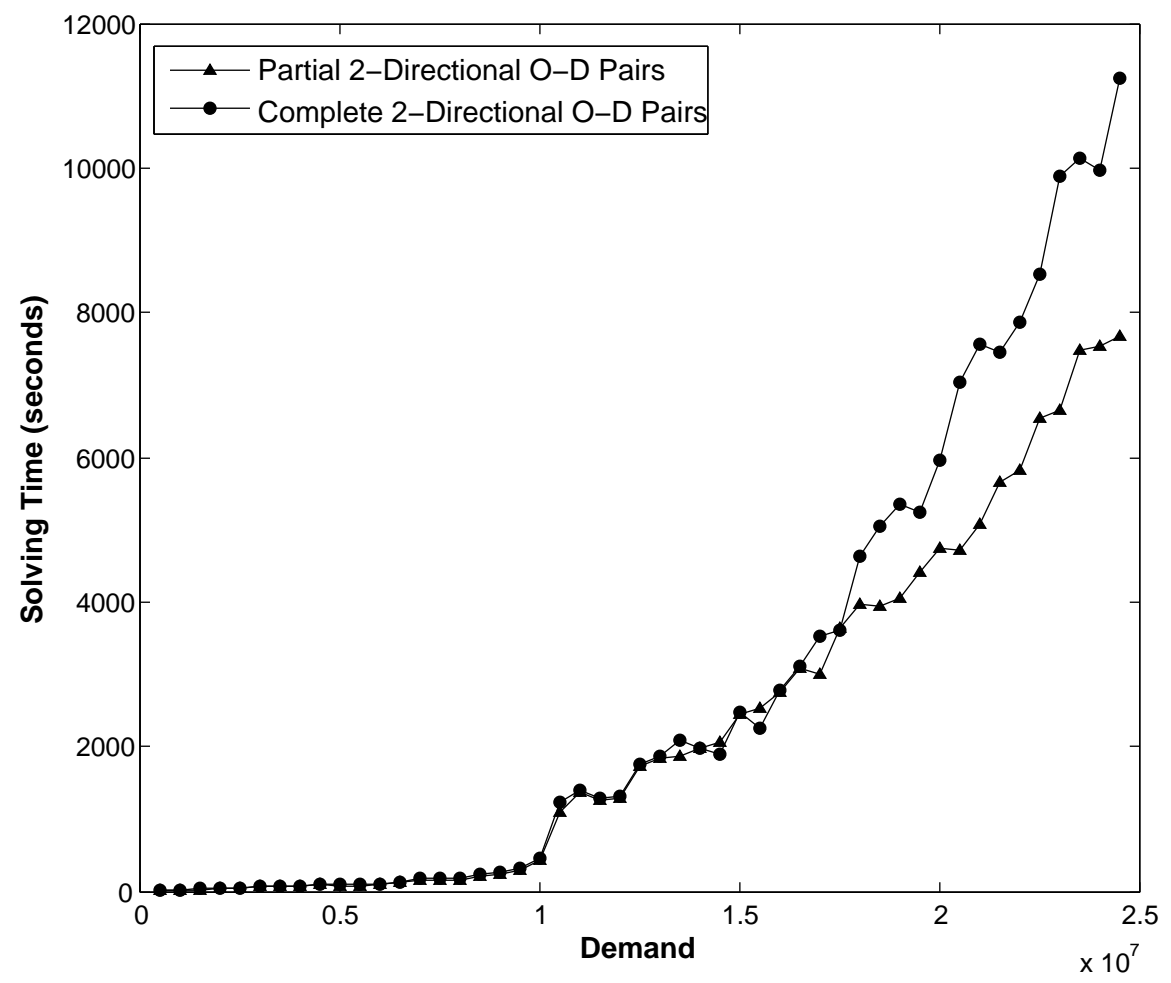

Figure 5.10: Partial and Complete 2-Directional O-D Pairs Solving Time vs. Demand. 


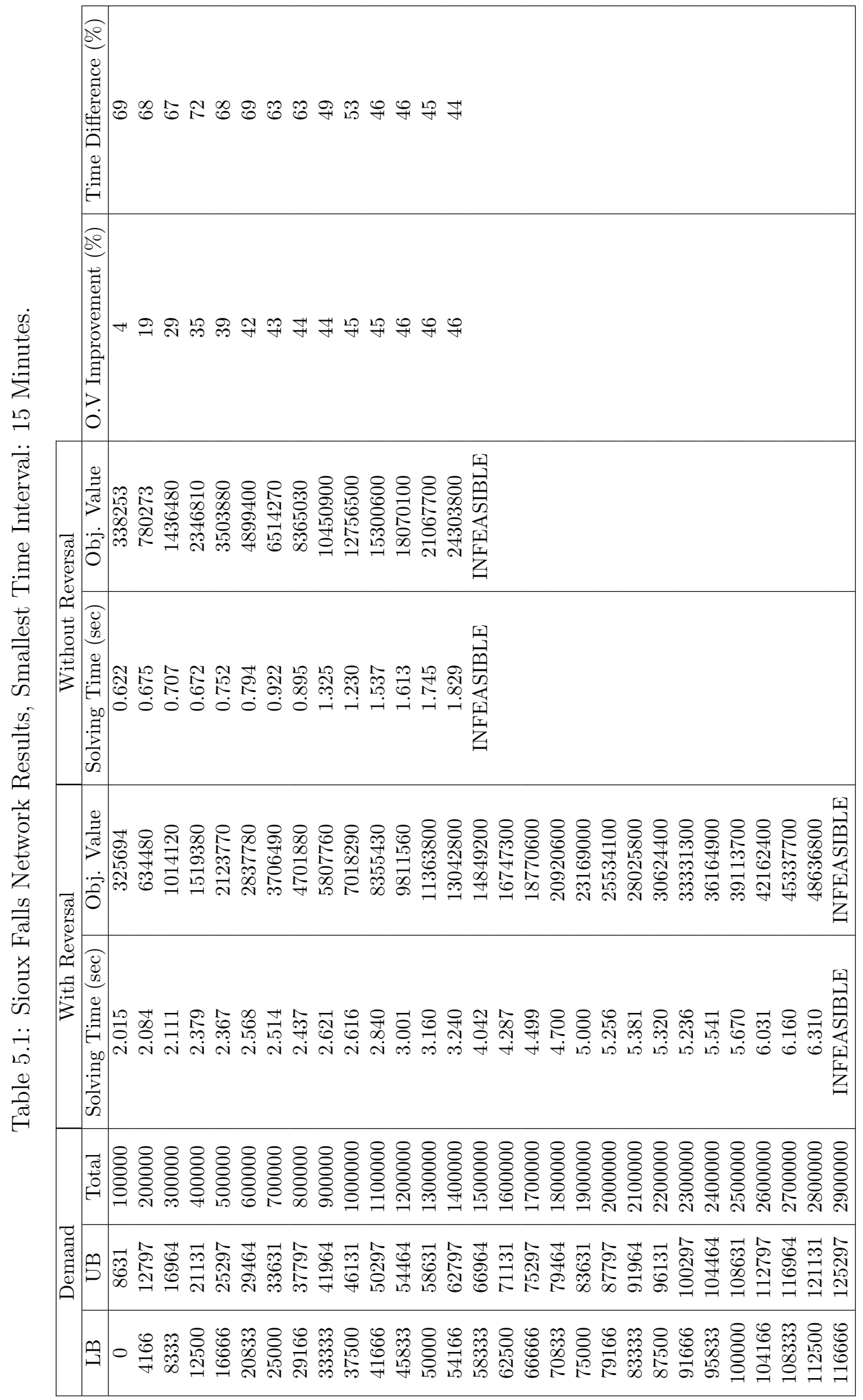




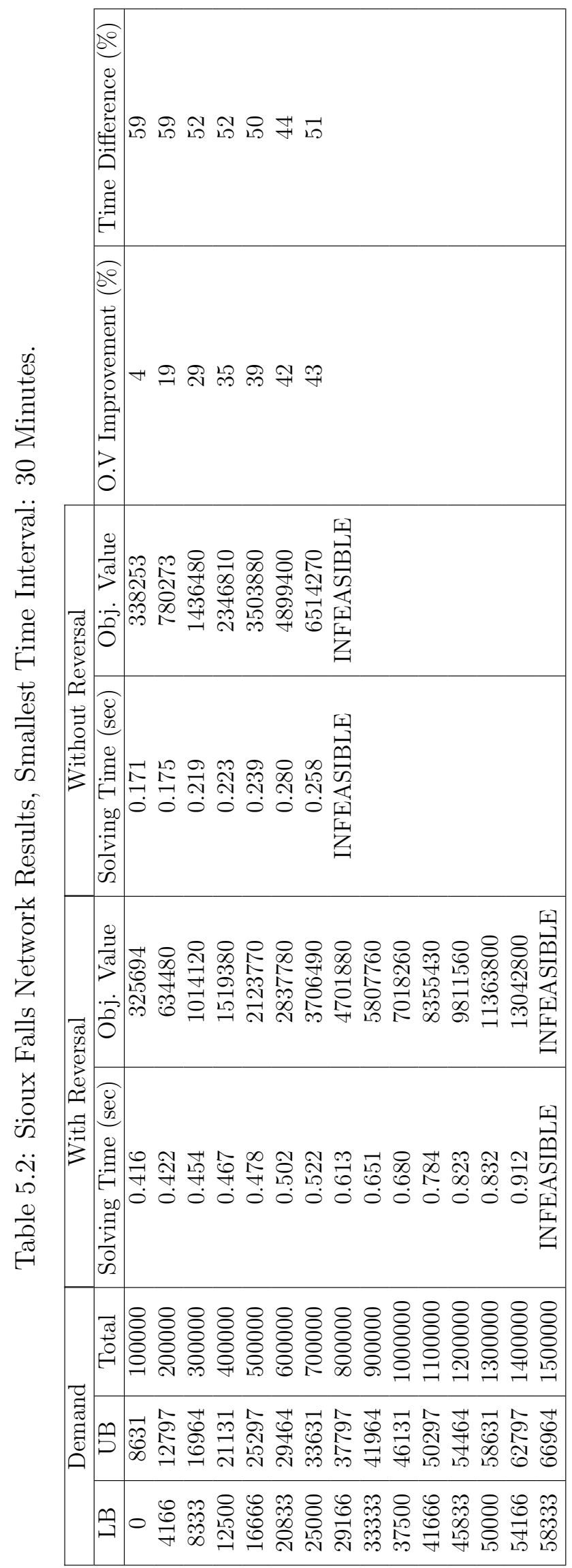




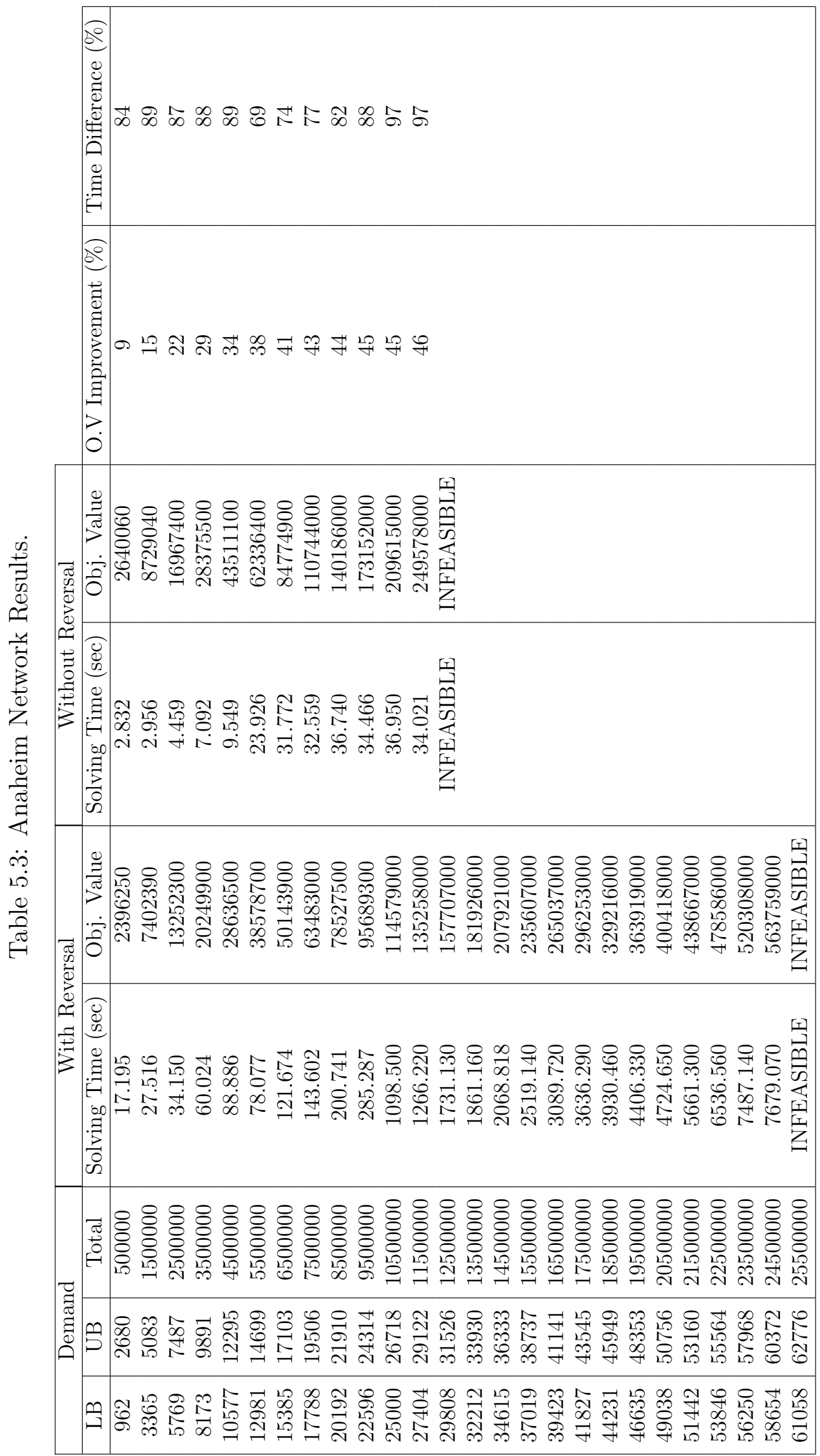


Table 5.4: Anaheim Network Results with Complete 2-Directional O-D Pairs.

\begin{tabular}{|c|c|c|c|c|}
\hline \multicolumn{3}{|c|}{ Demand } & \multicolumn{2}{c|}{ With Reversal } \\
\hline LB & UB & Total & Solving Time (sec) & Obj. Value \\
\hline 962 & 2680 & 500000 & 20.764 & 1846250 \\
3365 & 5083 & 1500000 & 31.085 & 6292390 \\
5769 & 7487 & 2500000 & 37.719 & 11262300 \\
8173 & 9891 & 3500000 & 70.289 & 17059900 \\
10577 & 12295 & 4500000 & 99.151 & 23926500 \\
12981 & 14699 & 5500000 & 88.342 & 32028700 \\
15385 & 17103 & 6500000 & 131.939 & 41433900 \\
17788 & 19506 & 7500000 & 169.296 & 52293000 \\
20192 & 21910 & 8500000 & 226.435 & 64537500 \\
22596 & 24314 & 9500000 & 310.981 & 78579300 \\
25000 & 26718 & 10500000 & 1224.194 & 94029000 \\
27404 & 29122 & 11500000 & 1291.914 & 110948000 \\
29808 & 31526 & 12500000 & 1756.824 & 129317000 \\
32212 & 33930 & 13500000 & 2086.854 & 149136000 \\
34615 & 36333 & 14500000 & 1894.512 & 170411000 \\
37019 & 38737 & 15500000 & 2244.834 & 193057000 \\
39423 & 41141 & 16500000 & 3115.414 & 217127000 \\
41827 & 43545 & 17500000 & 3596.123 & 242663000 \\
44231 & 45949 & 18500000 & 5032.336 & 269626000 \\
46635 & 48353 & 19500000 & 5236.214 & 298009000 \\
49038 & 50756 & 20500000 & 7036.471 & 327868000 \\
51442 & 53160 & 21500000 & 7456.214 & 359157000 \\
53846 & 55564 & 22500000 & 8524.336 & 391796000 \\
56250 & 57968 & 23500000 & 10142.214 & 420918000 \\
58654 & 60372 & 24500000 & 11253.144 & 451643686 \\
61058 & 62776 & 25500000 & 13755.354 & 480729607 \\
63462 & 65180 & 26500000 & 13854.789 & 509495529 \\
65866 & 67584 & 27500000 & 15963.324 & 537941450 \\
68270 & 69988 & 28500000 & 15632.254 & 566067371 \\
70674 & 72392 & 29500000 & 16987.567 & 593873293 \\
73078 & 74796 & 30500000 & 16874.364 & 621359214 \\
75482 & 77200 & 31500000 & 16963.125 & 648824836 \\
77886 & 79604 & 32500000 & INFEASIBLE & INFEASIBLE \\
\hline & & & & \\
\hline
\end{tabular}




\section{Chapter 6}

\section{Conclusions and Future Work}

Evacuation planning is of utmost importance for disaster prone areas. It provides authorities a solution to minimize the loss of lives. We formulated the emergency evacuation problem as a discrete-time system optimum DTA (DTA-SO) problem, which is a mixed integer nonlinear programming problem, and then linearized it to obtained a mixed integer linear programming problem, DTA-UB, which can approximate the original problem with very high precision. The discrete-time DTA model is suitable for evacuation planning for the reason that the model takes care of dynamic demands, and temporal flow assignment. Also, the model has simultaneous route and departure assumption, and uses appropriate travel time function. The main contribution of this research is the incorporation of lane reversals in to the DTA-UB model for tackling networks with multiple sources and multiple destinations. Lane reversals is the reversing of flow of a road segment from its regular direction. In other words, whole segments of roads are to flip its usual directional flow to head towards the opposite direction in order to increase outbound capacity in an evacuation. With the realization of lane reversals, naturally the threat of potential headon collisions emerges. To tackle this problem, we also incorporated a collision prevention constraint to limit the directional flow on lanes based on departure time.

To prove the effectiveness of our model, we formulated a no lane reversals DTA-UB model (NRDTA-UB) and compared its computational results with DTA-UB. DTA-UB proved to be able to generate better optimal solutions consistently across all demands. Furthermore, DTA-UB was able to optimize approximately double the amount of demand than that of NRDTA-UB.

Despite the advantage of better optimal solution, DTA-UB had significantly longer 
computational time than NRDTA-UB. Also, after a certain demand point, solving time of DTA-UB breaks its linear trend and jumps to a much higher value. Future research will need to be done to minimize this issue among others. Potential advance algorithms that can be implemented in the model to minimize the solving time of DTA-UB include Bender's Decomposition, Column Generation, Lagrangian Relaxation, and problem reformulation. Additional research can also consider partial evacuation of residents with restricted planning time horizon. Instead of eventually reaching infeasibility, the model focuses on evacuating as many residents as possible within the specified time horizon, and does not guarantee a 100\% evacuation of residents. Lastly, a capacity limit can be placed on all safe zones to model a more realistic scenario. In other words, there will be a limit to the number of evacuees entering a safe node and evacuees will be channeled to head towards other safe nodes once its capacity has reached its limit. 


\section{References}

[1] A. Stepanov and J. M. Smith, "Multi-objective evacuation routing in transportation networks," European Journal of Operational Research, vol. 198, no. 2, pp. 435-446, 2009.

[2] M. Lahmar, T. Assavapokee, and A. Ardekani, "A dynamic transportation planning support system for hurricane evacuation," in Intelligent Transportation Systems Conference, 2006. ITSC'06. IEEE. IEEE, 2006, pp. 612-617.

[3] M. Yusoff, J. Ariffin, and A. Mohamed, "Optimization approaches for macroscopic emergency evacuation planning: A survey," in Information Technology, 2008. ITSim 2008. International Symposium on, vol. 3. IEEE, 2008, pp. 1-7.

[4] Y. C. Chiu, J. Bottom, M. Mahut, A. Paz, R. Balakrishna, T. Waller, and J. Hicks, "Dynamic traffic assignment: A primer," Transportation Research E-Circular, no. E-C153, 2011.

[5] Q. P. Zheng and A. Arulselvan, "Discrete time dynamic traffic assignment models and solution algorithm for managed lanes," Journal of Global Optimization, vol. 51, no. 1, pp. 47-68, 2011.

[6] M. K. Lindell and C. S. Prater, "Critical behavioral assumptions in evacuation time estimate analysis for private vehicles: Examples from hurricane research and planning," Journal of Urban Planning and Development, vol. 133, no. 1, pp. 18-29, 2007.

[7] D. Guha-Sapir, F. Vos, R. Below, and S. Ponserre, "Annual disaster statistical review 2010," Centre for Research on the Epidemiology of Disasters, 2011.

[8] W. H. Hooke, "Us participation in international decade for natural disaster reduction," Natural Hazards Review, vol. 1, no. 1, pp. 2-9, 2000.

[9] R. T. Newkirk, "The increasing cost of disasters in developed countries: A challenge to local planning and government," Journal of Contingencies and Crisis Management, vol. 9, no. 3, pp. 159-170, 2002.

[10] C. Dybas and D. Terraso, "Number of category 4 and 5 hurricanes has doubled over the past 35 years," National Science Foundation, pp. 05-162, 2006. 
[11] Q. Lu, B. George, and S. Shekhar, "Capacity constrained routing algorithms for evacuation planning: A summary of results," Advances in Spatial and Temporal Databases, pp. 923-923, 2005.

[12] Y. M. Chen and D. Y. Xiao, "Real-time traffic management under emergency evacuation based on dynamic traffic assignment," in Automation and Logistics, 2008. ICAL 2008. IEEE International Conference on. IEEE, 2008, pp. 1376-1380.

[13] Y. C. Chiu, H. Zheng, J. Villalobos, and B. Gautam, "Modeling no-notice mass evacuation using a dynamic traffic flow optimization model," IIE Transactions, vol. 39, no. 1, pp. 83-94, 2007.

[14] M. S. Osman, B. Ram, J. Bhadury, P. Stanfield, L. Davis, and F. Samanlioglu, "Optimization model for distributed routing for disaster area logistics," in Service Operations, Logistics and Informatics, 2009. SOLI'09. IEEE/INFORMS International Conference on. IEEE, 2009, pp. 278-283.

[15] S. Kongsomsaksakul, C. Yang, and A. Chen, "Shelter location-allocation model for flood evacuation planning," Journal of the Eastern Asia Society for Transportation Studies, vol. 6, pp. 4237-4252, 2005.

[16] M. Saadatseresht, A. Mansourian, and M. Taleai, "Evacuation planning using multiobjective evolutionary optimization approach," European Journal of Operational Research, vol. 198, no. 1, pp. 305-314, 2009.

[17] Y. Liu, X. Lai, and G. L. Chang, "Two-level integrated optimization system for planning of emergency evacuation," Journal of transportation Engineering, vol. 132, no. 10 , pp. 800-807, 2006.

[18] A. Ben-Tal, S. R. Mandala, and T. Yao, "Evacuation under data uncertainty: robust linear programming model," in Transportation Research Board 88th Annual Meeting, no. 09-2779, 2009.

[19] Z. J. M. Shen, J. Pannala, R. Rai, and T. S. Tsoi, "Modeling transportation networks during disruptions and emergency evacuations," 2008.

[20] A. J. Pel, M. C. Bliemer, and S. P. Hoogendoorn, "A review on travel behaviour modelling in dynamic traffic simulation models for evacuations," Transportation, vol. 39, no. 1, pp. 97-123, 2012.

[21] O. L. Huibregtse, S. P. Hoogendoorn, A. Hegyi, and M. C. Bliemer, "A method to optimize evacuation instructions," OR spectrum, vol. 33, no. 3, pp. 595-627, 2011.

[22] C. Xie, D. Y. Lin, and S. Travis Waller, "A dynamic evacuation network optimization problem with lane reversal and crossing elimination strategies," Transportation Research Part E: Logistics and Transportation Review, vol. 46, no. 3, pp. 295-316, 2010. 
[23] D. Y. Lin, N. Eluru, S. T. Waller, and C. R. Bhat, "Evacuation planning using the integrated system of activity-based modeling and dynamic traffic assignment," Transportation Research Record: Journal of the Transportation Research Board, vol. 2132, no. 1, pp. 69-77, 2009.

[24] E. Stern and Z. Sinuany-Stern, "A behavioural-based simulation model for urban evacuation," Papers in Regional Science, vol. 66, no. 1, pp. 87-103, 1989.

[25] D. Helbing and A. Johansson, Pedestrian, crowd and evacuation dynamics. Swiss Federal Institute of Technology, 2009.

[26] H. Klpfel, T. Meyer-Knig, J. Wahle, and M. Schreckenberg, "Microscopic simulation of evacuation processes on passenger ships," in ACRI, vol. 2000, 2000, pp. 63-71.

[27] M. K. Lindell, "Emblem2: An empirically based large scale evacuation time estimate model," Transportation Research Part A: Policy and Practice, vol. 42, no. 1, pp. 140-154, 2008.

[28] S. K. Smith, J. Tayman, and D. A. Swanson, State and local population projections. Kluwer Academic, Plenum Publ., 2001.

[29] M. K. Lindell, C. S. Prater, and J. Y. Wu, Hurricane evacuation time estimates for the Texas Gulf Coast. Hazard Reduction \& Recovery Center, Texas A \& M University, 2002.

[30] S. W. Tweedie, J. R. Rowland, S. J. Walsh, R. P. Rhoten, and P. I. Hagle, "A methodology for estimating emergency evacuation times," The Social Science Journal, vol. 23, no. 2, pp. 189-204, 1986.

[31] M. Abkowitz and E. Meyer, "Technological advancements in hazardous materials evacuation planning," Transportation Research Record: Journal of the Transportation Research Board, vol. 1522, no. -1, pp. 116-121, 1996.

[32] T. J. Cova and J. P. Johnson, "Microsimulation of neighborhood evacuations in the urban-wildland interface," Environment and Planning A, vol. 34, no. 12, pp. 22112230, 2002.

[33] A. G. Hobeika, S. Kim, and R. E. Beckwith, "A decision support system for developing evacuation plans around nuclear power stations," Interfaces, vol. 24, no. 5, pp. 22-35, 1994.

[34] K. Dow and S. L. Cutter, "Emerging hurricane evacuation issues: hurricane floyd and south carolina," Natural hazards review, vol. 3, no. 1, pp. 12-18, 2002.

[35] D. J. Zeigler, S. D. Brunn, and J. H. Johnson Jr, "Evacuation from a nuclear technological disaster," Geographical review, pp. 1-16, 1981.

[36] T. E. Drabek, Disaster evacuation behavior: Tourists and other transients. Institute of Behavioral Science, University of Colorado Boulder, CO, 1996. 
[37] L. D. Han and F. Yuan, "Evacuation modeling and operations using dynamic traffic assignment and most desirable destination approaches," in the 84th Annual Meeting of Transportation Research Board, 2005.

[38] P. V. Hareesh, T. Hatanaka, and K. Sawada, "Simulation of crowd behavior during emergency evacuation based on artificial life," Human Media Technology Group, Advanced Technology Research Lab, Matsushita Electric Works, Ltd, 1998.

[39] D. Gunnec and F. Salman, "A two-stage multi-criteria stochastic programming model for location of emergency response and distribution centers," Euro-online. org, 2007.

[40] S. Shekhar and S. Kim, "Contraflow transportation network reconfiguration for evacuation route planning," Citeseer, Tech. Rep., 2006.

[41] B. George, S. Kim, and S. Shekhar, "Spatio-temporal network databases and routing algorithms: A summary of results," Advances in Spatial and Temporal Databases, pp. 460-477, 2007.

[42] R. Goldblatt, "Evacuation planning: A key part of emergency planning," in Proc., Transportation Research Board 83th Annual Meeting, 2004.

[43] A. K. Andreas, "Mathematical programming algorithms for reliable route and robust evacuation," 2006.

[44] N. Kamiyama, N. Katoh, and A. Takizawa, "An efficient algorithm for evacuation problems in dynamic network flows with uniform arc capacity," Algorithmic Aspects in Information and Management, pp. 231-242, 2006.

[45] E. Miller-Hooks and T. Krauthammer, "An intelligent evacuation, rescue and recovery concept," Fire technology, vol. 43, no. 2, pp. 107-122, 2007.

[46] A. H. F. Chow, "System optimal traffic assignment with departure time choice," Ph.D. dissertation, University of London, 2007.

[47] M. Beckmann, C. B. McGuire, and C. B. Winsten, "Studies in the economics of transportation," Tech. Rep., 1956.

[48] M. S. Bazaraa, H. D. Sherali, and C. M. Shetty, Nonlinear programming: theory and algorithms. Wiley-interscience, 2006.

[49] D. G. Luenberger and Y. Ye, Linear and nonlinear programming. Springer, 2008, vol. 116.

[50] P. Patriksson, The traffic assignment problem: models and methods. VSP, The Netherlands, 1994.

[51] J. G. Wardrop, "Some theoretical aspects of road traffic research," in Inst Civil Engineers Proc London/UK/, 1900. 
[52] T. L. Friesz, D. Bernstein, T. E. Smith, R. L. Tobin, and B. W. Wie, "A variational inequality formulation of the dynamic network user equilibrium problem," Operations Research, vol. 41, no. 1, pp. 179-191, 1993.

[53] D. K. Merchant and G. L. Nemhauser, "A model and an algorithm for the dynamic traffic assignment problems," Transportation Science, pp. 183-199, 1978.

[54] R. Balakrishna, Y. Wen, M. Ben-Akiva, and C. Antoniou, "Simulation-based framework for transportation network management in emergencies," Transportation Research Record: Journal of the Transportation Research Board, vol. 2041, no. -1, pp. 80-88, 2008.

[55] L. R. Ford and D. R. Fulkerson, "Constructing maximal dynamic flows from static flows," Operations Research, vol. 6, no. 3, pp. 419-433, 1958.

[56] R. E. Burkard, K. Dlaska, and B. Klinz, "The quickest flow problem," Mathematical Methods of Operations Research, vol. 37, no. 1, pp. 31-58, 1993.

[57] D. Gale, "Transient flows in networks," DTIC Document, Tech. Rep., 1958.

[58] B. Hoppe and . Tardos, "Polynomial time algorithms for some evacuation problems," in Proceedings of the fifth annual ACM-SIAM symposium on Discrete algorithms. Society for Industrial and Applied Mathematics, 1994, pp. 433-441.

[59] B. Hoppe and E. Tardos, "The quickest transshipment problem," Mathematics of Operations Research, vol. 25, no. 1, pp. 36-62, 2000.

[60] K. Jeannotte, A. Chandra, V. Alexiadis, and A. Skabardonis, "Traffic analysis toolbox volume ii: Decision support methodology for selecting traffic analysis tools," Tech. Rep., 2004.

[61] L. D. Han and F. Yuan, "Evacuation modeling and operations using dynamic traffic assignment and most desirable destination approaches," in 84th Annual Meeting of the Transportation Research Board, Washington, DC, 2005.

[62] A. J. Pel, M. C. Bliemer, and S. P. Hoogendoorn, "A review on travel behaviour modelling in dynamic traffic simulation models for evacuations," Transportation, vol. 39, no. 1, pp. 97-123, 2012.

[63] C. Xie and M. A. Turnquist, "Integrated evacuation network optimization and emergency vehicle assignment," Transportation Research Record: Journal of the Transportation Research Board, vol. 2091, no. 1, pp. 79-90, 2009.

[64] Y. Liu, X. Lai, and G. L. Chang, "Two-level integrated optimization system for planning of emergency evacuation," Journal of transportation Engineering, vol. 132, no. 10, pp. 800-807, 2006.

[65] T. Yao, S. R. Mandala, and B. Do Chung, "Evacuation transportation planning under uncertainty: a robust optimization approach," Networks and Spatial Economics, vol. 9, no. 2, pp. 171-189, 2009. 
[66] B. Wolshon, "one-way-out: contraflow freeway operation for hurricane evacuation," Natural hazards review, vol. 2, no. 3, pp. 105-112, 2001.

[67] P. B. Wolshon, E. A. Urbina, and M. Levitan, National review of hurricane evacuation plans and policies. Louisiana State University Hurricane Center Baton Rouge, 2001.

[68] G. L. Hamza-Lup, K. A. Hua, M. Lee, and R. Peng, "Enhancing intelligent transportation systems to improve and support homeland security," in Intelligent Transportation Systems, 2004. Proceedings. The 7th International IEEE Conference on. IEEE, 2004, pp. 250-255.

[69] G. Theodoulou, "Contraflow evacuation on the westbound i-10 out of the city of new orleans," Ph.D. dissertation, Faculty of the Louisiana State University and Agricultural and Mechanical College in partial fulfillment of the requirements for the degree of Master of Science in Civil Engineering in The Department of Civil and Environmental Engineering by Gregoris Theodoulou BS, Louisiana State University, 2003.

[70] G. Ford, R. Henk, and P. Barricklow, "Interstate highway 37 reverse-flow analysistechnical memorandum," Prepared for the Texas Department of Transportation, Texas Transportation Institute, San Antonio, Tex, 2000.

[71] A. Nahapetyan and S. Lawphongpanich, "Discrete-time dynamic traffic assignment models with periodic planning horizon: system optimum," Journal of Global Optimization, vol. 38, no. 1, pp. 41-60, 2007.

[72] H. C. Manual, "Highway capacity manual," 2000.

[73] M. M. Minderhoud, H. Botma, and P. H. Bovy, "Assessment of roadway capacity estimation methods," Transportation Research Record: Journal of the Transportation Research Board, vol. 1572, no. 1, pp. 59-67, 1997.

[74] G. E. Box and N. R. Draper, "Empirical model-building and response surfaces," 1987.

[75] H. Bar-Gera, "Transportation network test problems," 2002. 